\title{
WSRC-TR-2002-00219
}

KEYWORDS:

Solid Suspension

Computational Approach CFD Approach

Agitator Mixing Model

Turbulence Model

RETENTION - Permanent

\section{AGITATOR MIXING ANALYSIS IN A HB-LINE FLAT TANK}

SAVANNAH RIVER TECHNOLOGY CENTER

Si Young Lee

Publication Date: May 2002

Westinghouse Savannah River Company

Savannah River Site

Aiken, SC 29808

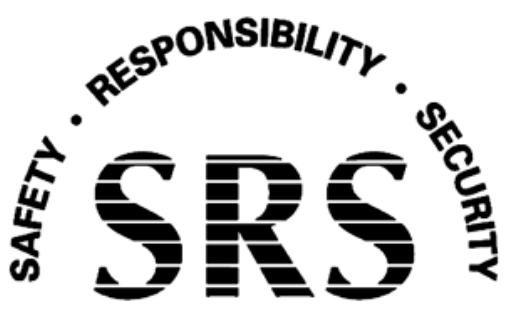

SAVANNAH RIVER SITE

Prepared for the U.S. Department of Energy under Contract No. DE-AC09-96SR18500 
This document was prepared in conjunction with work accomplished under Contract No. DE-AC09-96SR18500 with the U. S. Department of Energy.

\section{DISCLAIMER}

This report was prepared as an account of work sponsored by an agency of the United States Government. Neither the United States Government nor any agency thereof, nor any of their employees, makes any warranty, express or implied, or assumes any legal liability or responsibility for the accuracy, completeness, or usefulness of any information, apparatus, product or process disclosed, or represents that its use would not infringe privately owned rights. Reference herein to any specific commercial product, process or service by trade name, trademark, manufacturer, or otherwise does not necessarily constitute or imply its endorsement, recommendation, or favoring by the United States Government or any agency thereof. The views and opinions of authors expressed herein do not necessarily state or reflect those of the United States Government or any agency thereof.

This report has been reproduced directly from the best available copy.

Available for sale to the public, in paper, from: U.S. Department of Commerce, National Technical Information Service, 5285 Port Royal Road, Springfield, VA 22161, phone: (800) 553-6847, fax: (703) 605-6900

email: orders@ntis.fedworld.gov

online ordering: http://www.ntis.gov/help/index.asp

Available electronically at http://www.osti.gov/bridge

Available for a processing fee to U.S. Department of Energy and its contractors, in paper, from: U.S. Department of Energy, Office of Scientific and Technical Information, P.O. Box 62, Oak Ridge, TN 37831-0062,

phone: (865)576-8401,

fax: (865)576-5728

email: $\underline{\text { reports@ adonis.osti.gov }}$ 
DOCUMENT: WSRC-TR-2002-00219

TITLE: $\quad$ AGITATOR MIXING ANALYSIS IN A HB-LINE FLAT TANK

APPROVALS
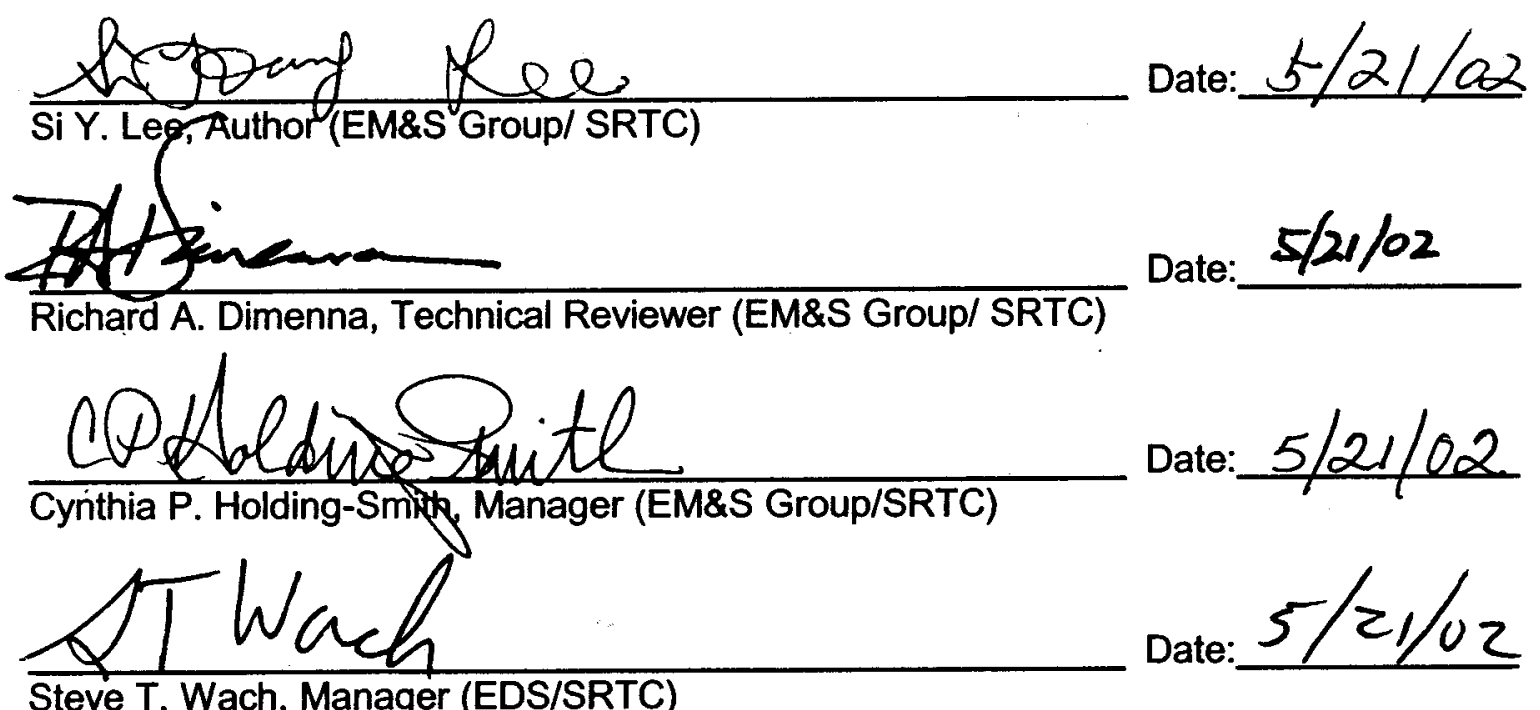

-iii- 
(This Page Intentionally Left Blank) 


\section{Table of Contents}

List of Figures $\quad$ vii

List of Tables $\quad x$

Nomenclature $\quad$ xi

$\begin{array}{ll}\text { Abstract } & 1\end{array}$

1 Introduction 2

2. Modeling Approach and Analysis $\quad 5$

3. Results and Discussions 13

4. Conclusions 53

5. References $\quad 55$ 
(This Page Intentionally Left Blank) 


\section{List of Figures}

Figure 1. Geometry of the agitator tank with pitched blades used for the modeling analysis

Figure 2. Configurations of the agitator with three 45-deg pitched blades used for the model.

Figure 3. Static forces for a solid particle in a flowing fluid assuming cohesionless particle layer.

Figure 4. Air pull-through entrainment due to a large axial convective downward flow induced by high rotational speed of pitched agitator blade submerged in a low tank level.....

Figure 5. Geometrical configurations for 45-deg pitched three-blade agitator submerged in a flat tank as modeled for the present analysis.

Figure 6. Three-dimensional unstructured hybrid meshes for the flat tank with 45-deg pitched three-blade agitator as modeled for the present analysis.

Figure 7. Maximum fluid velocity as function of the rotational speed of $45^{\circ}$ pitched agitator submerged in flat tank.

Figure 8. Average local velocity versus the product parameter of the rotational speed $(\mathrm{N})$ and blade diameter $\left(D_{B}\right)$ for three-blade $45^{\circ}$ pitched agitator submerged in flat tank. The average velocity was obtained by averaging local velocities along the line A-A'....

Figure 9. Average local fluid velocity at the corner of tank bottom versus the parameter

$(\theta)$ related to mixing performance (Slope of the linear fit is about 0.54.) The average velocity was obtained by averaging local velocities along the line A-A' ..20

Figure 10. Velocity contour plot at the plane A-A' of the mixing tank with $300 \mathrm{rpm}$ agitator speed and 12-inch liquid level.

Figure 11. Velocity contour plot at the plane A-A' of the mixing tank for the reference case shown in Table 1 (12-in tank level and 500 rpm agitator speed)

Figure 12. Velocity contour plot at the plane A-A' of the mixing tank with 12 -inch liquid level and $700 \mathrm{rpm}$ agitator speed.

Figure 13. Average local velocity magnitude versus the parameter (ND) of the agitator speed for various distances from the agitator submerged in 12-inch tank level ...24

Figure 14. Shear stress contour plot at the wall surface of mixing tank for different speeds of the agitator submerged in 12-inch tank level showing the red zone to be larger than critical shear stress for 20 micron particle.

Figure 15. Pressure contour plot on the horizontal plane crossing the blade of the agitator (plane B-B') submerged in a flat tank for the reference case shown in Table 1 (12-in tank level and agitator speed $=500 \mathrm{rpm}$ )

Figure 16. Velocity vector plot on the horizontal plane crossing the blade for the reference case shown in Table 1 (12-in tank level and agitator speed $=500$ rpm).

Figure 17. Velocity vector plot in three-dimensional fluid domain for the reference case shown in Table 1 (12-in tank level and agitator speed $=500 \mathrm{rpm}$ ).

Figure 18. Three-dimensional velocity vector plot near the agitator blade from the top of the tank for the reference case shown in Table 1 (12-in tank level and agitator speed $=500 \mathrm{rpm}$ ). 
Figure 19. Velocity vector plot near the agitator blade at the mid-plane of the tank (plane A-A') for the reference case shown in Table 1 (12-in tank level and agitator speed $=500 \mathrm{rpm})$.......

Figure 20. Velocity distributions at the vertical plane crossing the line A-A' for three different agitator speeds in the flat tank filled with 12-inch liquid level....

Figure 21. Wall shear distributions on the surface of the agitator blade and its rotational axis located near the tank bottom for the reference case shown in Table 1 (12-in tank level and 500 rpm agitator speed)

Figure 22. Contour plots for some physical parameters due to the clockwise rotations of the agitator on the plane crossing the vertical center of the agitator for the reference case (500 rpm agitator speed and 12-inch tank level)

Figure 23. Distributions of physical parameters associated with flow circulation pattern and mixing characteristics for the plane crossing the center of the $500 \mathrm{rpm}$ mixing agitator submerged in the tank with 8.5-inch liquid level.

Figure 24. Velocity contour plots for the horizontal planes corresponding to different elevations above the tank bottom for $500 \mathrm{rpm}$ agitator speed and the 8.5-inch tank level.

Figure 25. Velocity vector plots for the planes at several different distances $(x=2$ ", 8.25 ", 17.5", 23.5", and 27.5") from the agitator located at the center of the flat tank (500 rpm agitator speed with 8.5-inch tank level).

Figure 26. Turbulence intensity contour plots for the planes at several different distances ( $x=2$ ", 8.25", 17.5", 23.5", and 27.5") from the agitator located at the center of the flat tank (agitator speed $=500 \mathrm{rpm}$ and 8.5-inch tank level)....

Figure 27. Velocity contour plots for the planes at several different distances $(x=2 "$, $8.25 ", 17.5 ", 23.5 "$, and 27.5 ") from the agitator located at the center of the flat tank with 8.5-inch liquid level.

Figure 28. Velocity contour plots at the vertical plane crossing the mixer (line A-A') for various different agitator speeds in the flat tank filled with 8.5-inch liquid level .....39

Figure 29. Three-dimensional velocity contour plots showing the fluid domain to be higher than minimum critical velocity $(0.225 \mathrm{ft} / \mathrm{sec}$ for 20 -micron particle) for flat tank with $600 \mathrm{rpm}$ agitator speed and 8.5-inch tank level.

Figure 30. Velocity distributions at the vertical plane crossing the line A-A' for three different agitator speeds in the flat tank filled with 8.5-inch liquid level

Figure 31. Velocity distributions along the vertical direction from the tank bottom for various horizontal distances from the 700-rpm agitator submerged in the flat tank filled with 8.5-inch liquid level.

Figure 32. Velocity contour plots at the vertical plane crossing the mixer (line A-A') for various different agitator speeds in the flat tank filled with 7-inch liquid level

Figure 33. Velocity distributions along the vertical line A-A' for various different agitator speeds at the horizontal distance of 23.5 inches from the agitator submerged in the flat tank filled with 7-inch liquid level

Figure 34. Velocity distributions along the vertical line A-A' for two different agitator speeds at the horizontal distance of 23.5 inches from the agitator submerged in the flat tank filled with 4.5-inch liquid level.

Figure 35. Velocity contour plots at the vertical plane crossing the mixer (line A-A') for two different agitator speeds in the flat tank filled with 4.5-inch liquid level

Figure 36. Velocity distributions along the vertical line A-A' for $500 \mathrm{rpm}$ agitator speed at the horizontal distance of 23.5 inches from the agitator submerged in the flat tank filled with various liquid levels. 
Figure 37. Velocity distributions along the vertical line A-A' for $700 \mathrm{rpm}$ agitator speed at the horizontal distance of 23.5 inches from the agitator submerged in the flat tank filled with various liquid levels

Figure 38. Typical transient results for water volume fractions in 4.5-inch tank level with $400 \mathrm{rpm}$ agitator speed considering free surface motion

Figure 39. Flow circulation patterns and characteristics for high and low tank levels with turbulent $45^{\circ}$ pitched agitator submerged.

Figure 40. Primary flow components induced by the turbulent agitator submerged in a tank 


\section{List of Tables}

Table 1...Operating Conditions used for the modeling and analysis ...................................10

Table 2. Flow conditions driven by the agitator shown in Fig. 1 .......................................10

Table 3. Critical velocities for various liquid levels to initiate the movement for 20-micron solid particles deposited at the bottom of flat tank ..................................................17

Table 4. Conservative estimation of liquid level for the inception of air pull-through .17

Table 5. Average local velocity magnitudes for various agitator speeds at two different distances (18 inches and 23.5 inches) from the agitator submerged in 12-inch tank level.

Table 6. Modeling results for various tank levels and pump speeds compared with critical velocities. 


\section{Nomenclature}

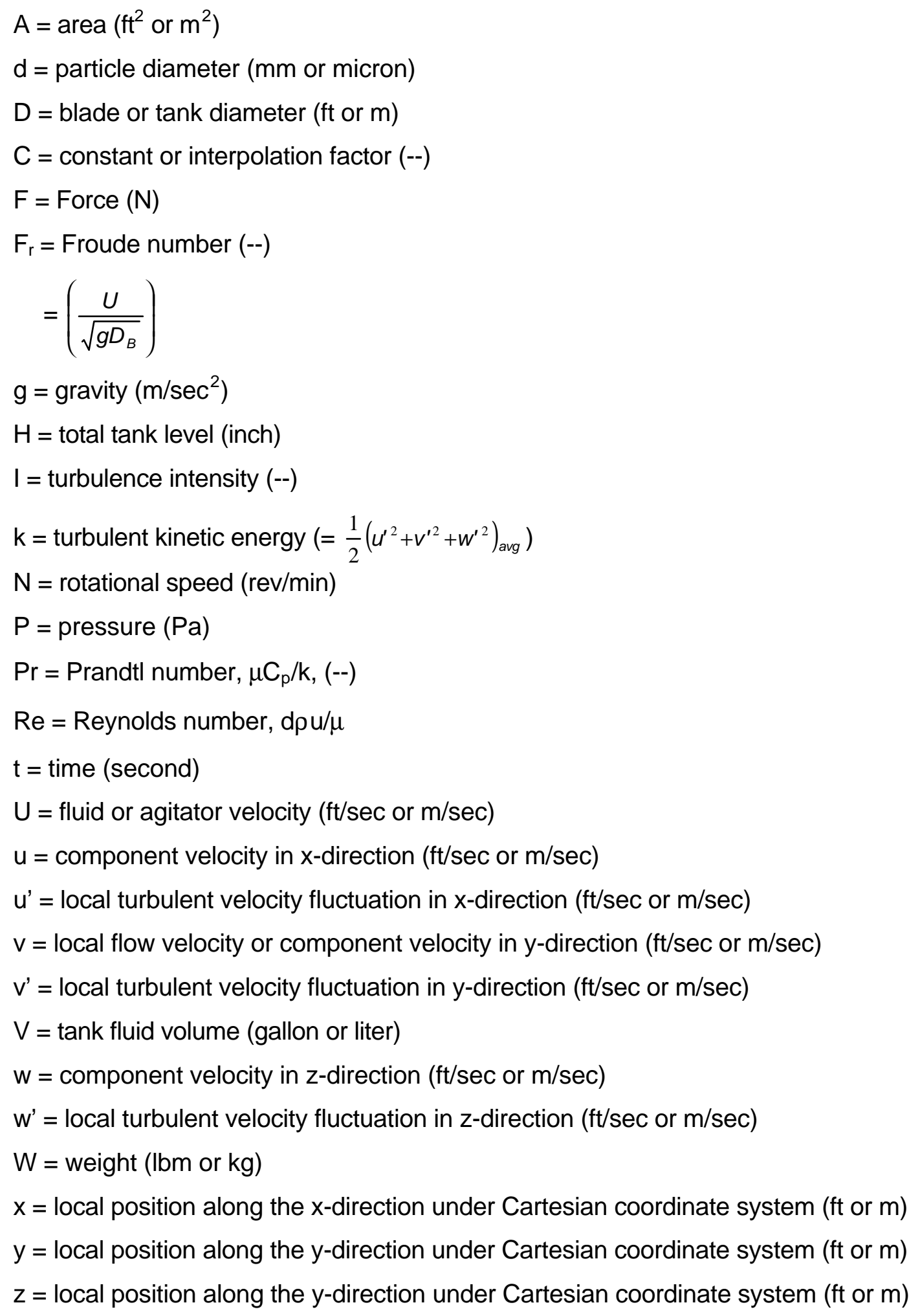




\section{Greek}

$\rho=$ density $\left(\mathrm{kg} / \mathrm{m}^{3}\right)$

$\varepsilon=$ rate of dissipation of turbulent kinetic energy

$\nabla=$ gradient operator

$\omega=$ vorticity

$\zeta=$ solid weight per weight of liquid

$\mu=$ dynamic viscosity $\left(\mathrm{N} \mathrm{sec} / \mathrm{m}^{2}\right)$

$\tau=$ shear stree $\left(\mathrm{Pa}\right.$ or $\left.\mathrm{N} / \mathrm{m}^{2}\right)$

$v=$ kinematic viscosity $\left(\mathrm{m}^{2} / \mathrm{sec}\right)$

$\theta=$ velocity parameter (ft/sec or $\mathrm{m} / \mathrm{sec}$ )

\section{$\underline{\text { Subscript }}$}

$a=$ agitator

avg $=$ average

$\mathrm{B}=$ agitator blade

$\mathrm{f}=$ fluid

fs = suspended fluid

$\mathrm{L}=$ lifting

$\mathrm{m}=$ maximum

$p=$ particle

$\mathrm{S}=$ static

$\mathrm{T}=$ tank

$w c=$ critical wall shear 


\section{Abstract}

In support of the HB-Line Engineering agitator mixing project, flow pattern calculations have been made for a $45^{\circ}$ pitched three-blade agitator submerged in a flat rectangular tank. The work is intended to determine agitator speeds that provide acceptable mixing performance for various tank liquid levels based on flow rates past solids deposited on the bottom surface of the flat tank. The modeling results will help ensure the acceptable suspension of solid particles as a function of agitator speed and tank liquid level during precipitation operations.

The numerical modeling and calculations have been performed using a computational fluid dynamics approach. Three-dimensional steady-state momentum and continuity equations were used as the basic equations to estimate fluid motion driven by an agitator with three $45^{\circ}$ pitched blades. Hydraulic conditions were fully turbulent (Reynolds number about $2 \times 10^{4}$ ). A standard two-equation turbulence model $(\kappa-\varepsilon)$, was used to capture turbulent eddy motion. The commercial finite volume code, Fluent [7], was used to create a prototypic geometry file with a non-orthogonal mesh. Hybrid meshing was used to fill the computational region between the round-edged tank bottom and agitator regions.

At high rotational speeds and low tank levels, a surface vortex can reach the agitator blades and allow air to be drawn into the solid-fluid mixing zone. This is not desirable in terms of mixing performance. The analysis results show that the lowest liquid level among the four considered, 4.5 inches, is higher than the critical liquid height for air entrainment for agitator speeds up to $600 \mathrm{rpm}$.

All the analysis results demonstrate that about $600 \mathrm{rpm}$ provides adequate solids mixing capability for various tank levels $(12,8.5,7$, and 4.5 inches) containing 20-micron solids with a specific gravity of 2.5 . 


\section{Introduction}

The present analysis considers a stationary solids layer on the bottom surface of the HBline agitator tank consisting of loose and noncohesive solid particles of uniform size, and liquid flowing over it. This condition will typically occur during a precipitation operation after being mixed by a mechanical agitator. Liquid flow and the deposited solid layer are assumed to be statistically in a steady state so that the local flow velocity can be considered the initiator for movement of a solid particle located on the top of the solid layer. As soon as liquid starts flowing, hydrodynamic forces are exerted upon the solid particles resting along the wetted perimeter of the fluid-solid system. An increase in flow velocity causes an increase in the magnitude of these forces. Eventually, a condition is reached at which particles in the movable layer are unable to resist the hydrodynamic forces, first get dislodged, and then start to move. In reality, some move and some do not for any given hydraulic condition. The statistical nature of this behavior points to the fact that the flow is turbulent. The onset of initial movement of the solids layer is predicted by an interfacial force balance on the fluid-solid system.

As requested by the customer [1], the objective of the present work is to:

- Determine an efficient agitator speed and tank liquid level to improve mixing flow over solids deposited on the tank bottom surface.

- Evaluate minimum liquid level to avoid drawing air into the agitator blade and degrading mixing performance.

- Provide guidance for acceptable mixing in the HB-line tank.

The primary goal of the present work is to estimate an agitator speed and tank liquid level which provide acceptable mixing performance with a $45^{\circ}$ pitched three-blade agitator. The results will also help optimize solid suspension during precipitation.

Schematic diagrams for the HB-line mixing tank system and geometrical configurations for the agitator are illustrated in Figs. 1 and 2 as provided by the customer [1, 2]. 

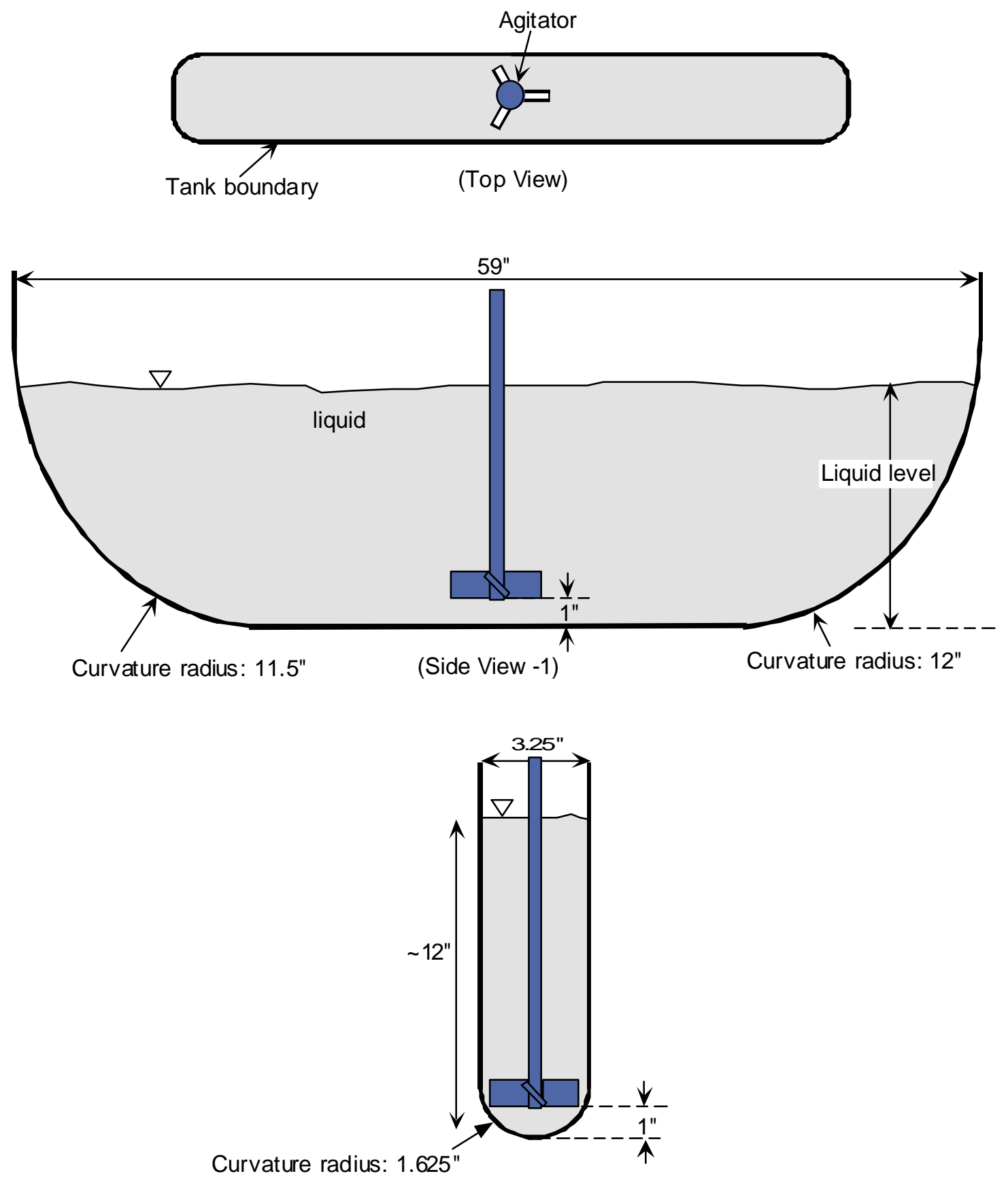

(Side View -2)

Figure 1. Geometry of the agitator tank with pitched blades used for the modeling analysis 

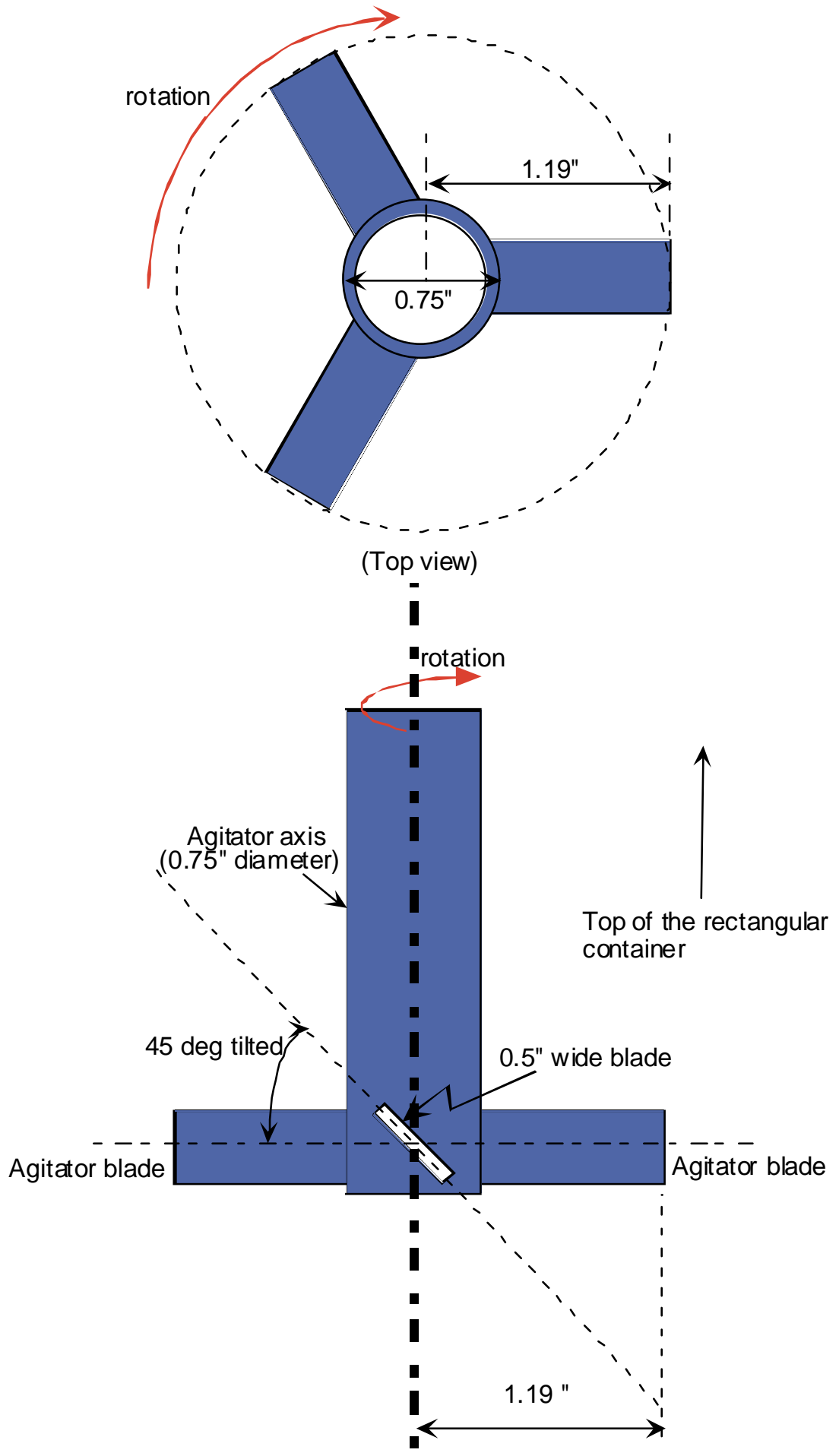

(Side view)

Figure 2. Configurations of the agitator with three $45^{\circ}$ pitched blades used for the model 


\section{Modeling Approach and Analysis}

The tank has a thin, flat and rectangular shape. It is about 60" long and 3.25 " wide. The tank bottom has round-edged corners with 11.5" and 12" curvature radii at either end of the flat sides and a 1.625" curvature radius for the corner of the round sides. Detailed geometrical configurations of the mixing tank are shown in Fig. 1.

The initial movement of the solids deposited at the bottom of the tank identifies the critical condition or initial scour. It is usually described by two mechanistic criteria:

- The critical velocity criterion considers the impact of the liquid motion on the particle surface such as lifting force due to the gradient of the velocity around the particles.

- The critical shear stress criterion considers the frictional drag of the flow on the particles.

Information gained here will be employed in determining critical conditions to initiate the movement of deposited solids. From these two criteria, the one to provide conservative estimation will be chosen as a performance criterion for the operating guidance of the adequate solid mixing under the present geometrical configurations.

\section{A. Critical Velocity Criterion}

The mechanism of particle lifting or pickup in the vicinity of a horizontal surface involves the fluid velocity required to pick up, or prevent deposition of, particles of a particular size and density. The pickup or suspension velocity for a solid particle lying on a solid horizontal surface, as shown in Fig. 3, is evaluated from a balance of forces induced by or associated with a horizontal flow of fluid.

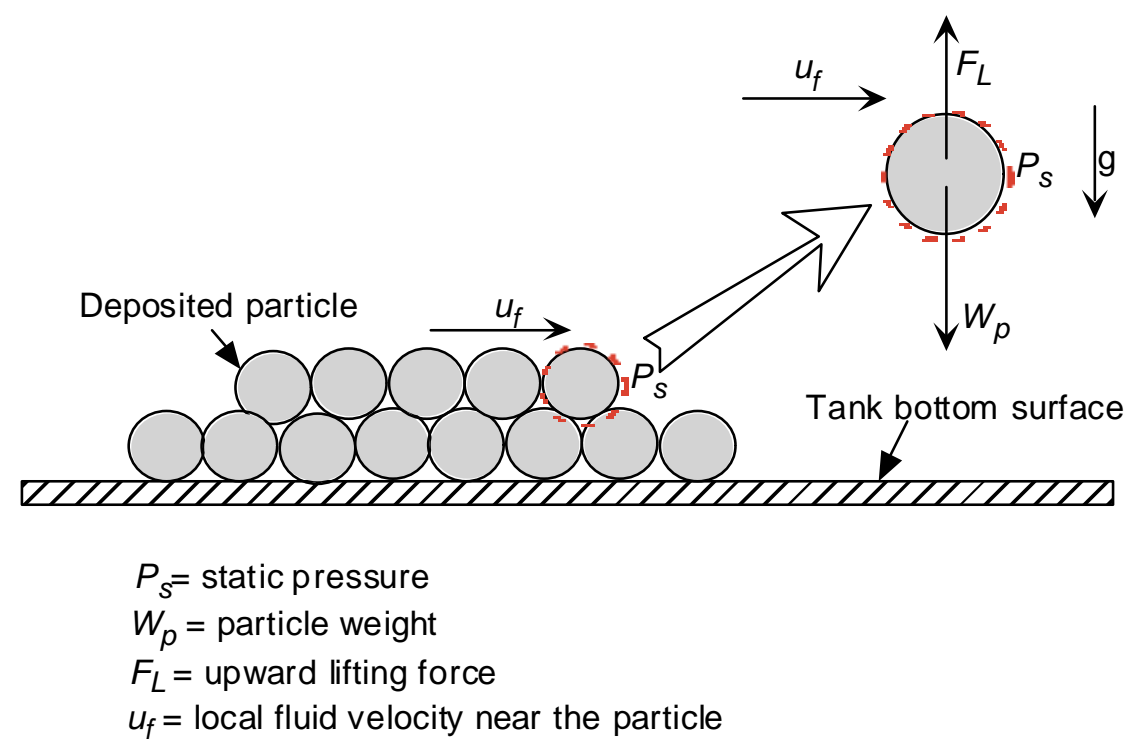

Figure 3. Static forces for a solid particle in a flowing fluid assuming a noncohesive particle layer. 
Date: $\quad 07 / 24 / 02$

Page: $\quad 6$ of 56

The condition of incipient movement for an assembly of noncohesive, loose, solid particles is described in terms of the forces acting on the particle. The driving force for initial movement is the resultant of the hydrodynamic lifting force, $F_{L}$, and the submerged weight of particle, $W_{p}$, for a given hydrostatic pressure, $P_{\mathrm{s}}$.

A particle such as shown in Fig. 3 is subject to an upward lifting force $F_{L}$, the Bernoulli force acting on the particle's projected area $\left(A_{p}\right)$ where

$$
F_{L}=\left(P_{s}+\frac{1}{2} \rho_{f} u_{f}^{2}\right) A_{p},
$$

and a downward force equal to the static pressure $P_{s}$ and the particle weight,

$$
F_{D}=P_{s} A_{p}+V_{p}\left(\rho_{p}-\rho_{f}\right) g
$$

Hydrostatic pressure acts on both the top and bottom of the particle. At the point at which the particle would just begin to rise, the upward force equals the downward force, so that equation (1) equals equation (2). For spherical particles, the ratio of $V_{p}$ to $A_{p}$ is equal to $2 d_{p} / 3$, so that the above relationship simplifies to

$u_{f}=\sqrt{\frac{4}{3} g d_{p}\left(\frac{\boldsymbol{\rho}_{p}}{\boldsymbol{\rho}_{f}}-1\right)} \approx 3.617 \sqrt{d_{p}\left(\boldsymbol{s}_{p}-1\right)}$.

Rotational motion of the particle is negligible since the particle is small (about 10 to 20 microns). It should be noted that the model neglects inter-particle cohesion and static friction between the surface and a particle at rest based on an assumption that solids submerged within an open tank are loosely deposited during precipitation. In theory, the velocity required to overcome friction and cohesion can be considerably greater than that derived from this model, which accounts solely for weight and lifting force due to fluid momentum.

Experimental studies of scour criteria have been published in the literature. Graf (1971) presented an empirical correlation for the critical velocity required to initiate movement of a deposited particle. In the correlation, he used average fluid velocity rather than the bottom velocity, since most of the previous works on erosion studies used average velocity. The empirical correlation for critical velocity $\left(U_{c}\right)$ was made in terms of particle size $\left(d_{p}\right)$, liquid level $\left(H_{\ell}\right)$, and specific gravity $\left(s_{p}\right)$ of solid particle.

$U_{c}=\left(\frac{H_{\ell}}{d_{p}}\right)^{0.1} \sqrt{2.5 g d_{p}\left(\frac{\rho_{p}}{\rho_{f}}-1\right)}=4.9523\left(\frac{H_{\ell}}{d_{p}}\right)^{0.1} \sqrt{d_{p}\left(S_{p}-1\right)}$

Equation (4) was established with test data from various investigations. It is functionally similar to the theoretical formulation of equation (3), since the liquid level parameter is only weakly related to the critical velocity. Because of its empirical nature, equation (4) includes the influence of friction and cohesive forces. This, combined with its development in terms of average fluid velocity, is reflected in the larger coefficient than seen in equation (3). To apply equation (4) to the present work, it is assumed that for each grain size, a certain "critical velocity" exists, below which the particle will experience sedimentation, and above which it will be lifted or eroded from the solid layer. The 
interpretations of flow patterns were based on 20-micron particles, since the critical velocity for a 20 -micron particle is larger than that for 10 -micron particle.

\section{B. Critical Shear Stress Criterion}

The stress on tank wall due to shearing action of a flowing fluid was thought to be an important feature of initial scour or the critical condition for initial movement of a deposited particle. The abrasive friction due to wall shear is the key mechanism for the initial scour of particles from the wall surface. Given the small particle size in the present operating conditions, other influences such as particle-particle interactions and the effects of the particles on the continuous liquid phase are negligible.

The turbulent fluid-wall interaction is evaluated with a standard $\kappa-\varepsilon$ model. This model computes the turbulent eddy viscosity $v_{t}$ by solving two transport equations for $\kappa$ (turbulent kinetic energy) and $\varepsilon$ (rate of dissipation of turbulent energy). The literature shows that the turbulent eddies are constantly lifting particles into the fluid stream due to the fluctuations in velocity [3]. When applying the wall shear mechanism to the mixing evaluation, it is assumed that the wall shear stress affects the initial scour or lifting-up of the solids lying at the bottom of the agitator tank.

Graf (1971) recommended an empirical correlation for critical shear stress $\left(\tau_{w c}\right)$ to initiate the movement of deposited particles. In his correlation, he used mean particle size $\left(d_{p}\right)$ as key parameter. A convincing shear stress versus grain size relationship could be represented with the simple relationship,

$\tau_{w c}=0.166 g\left(d_{p}\right)_{\text {avg }} \approx 1.6285\left(d_{p}\right)_{\text {avg }} \quad\left(N / m^{2}\right.$ or $\left.\mathrm{Pa}\right)$

Equation (5) was developed from various sources of data available in the literature. It applies to solid particles less than $3.4 \mathrm{~mm}$ in diameter. The mean particle diameter in equation (5) is in millimeters.

\section{Modeling Approach}

Based on the performance criteria discussed earlier, both steady-state and transient computational approaches were used to compute flow fields driven by agitator. Most analyses were based on the steady-state model for computational efficiency. Some transient model calculations were performed as scoping analyses to evaluate free surface motion at the top of the HB-line flat tank. The main solution methodologies and modeling assumptions were as follows:

- A computational fluid dynamics (CFD) approach was taken, using the commercial code, Fluent $^{\mathrm{T} M}$.

- A prototypic geometry for the agitator and mixing tank was created with Fluent using a non-orthogonal control volume method.

- The present model was based on the $45^{\circ}$ pitched three-blade agitator and tank with no internal solid structures. 
- The mixing simulations used three-dimensional steady-state, isothermal governing equations with multiple reference frames (MRF).

- For the steady-state model, the top liquid surface was assumed to be stationary and at atmospheric pressure.

- A two-equation standard turbulence model was used.

As mentioned above, the steady-state model assumes that free surface remains stationary at atmospheric pressure. If the agitator rotates in a clockwise (as viewed from above), a large axial convection flow moves downward due to the rotation of the pitched blade. When liquid level becomes low enough to get air pull-through due to vortex formation near the tips of the agitator blades, air will be drawn into the blade zone as shown in Fig. 4.

An empirical equation (Schrock et al, 1985) is available in the literature to estimate the minimum liquid level which prevents air entrainment into the blade zone of the agitator. They authors correlated the equation for critical liquid level $\left(H_{c}\right)$ in terms of Froude number $\left(F_{r}\right)$ and fluid properties to get air pull-through from the free surface to the liquid region. The empirical equation for the critical liquid height is

$$
\begin{aligned}
H_{c}=0.8513 D_{B}\left(F_{r}\right)^{0.5}\left(\frac{\rho_{f}}{\rho_{f}-\rho_{a}}\right)^{0.25} \\
\text { where } F_{r}=\left(\frac{U}{\sqrt{g D_{B}}}\right) \\
\mathrm{U}=\text { Maximum fluid velocity } \\
D_{\mathrm{B}}=\text { blade diameter } \\
\rho_{\mathrm{f}}=\text { liquid density } \\
\rho_{\mathrm{a}}=\text { air density }
\end{aligned}
$$

The analysis consists of two major parts. One part is to calculate the reference operating conditions (12-inch tank level and $500 \mathrm{rpm}$ agitator speed) to ensure that the model is representative of actual flow patterns during mixing operations. The second part is to apply the model to mixing simulations and flow pattern analysis for various tank levels and agitator speeds to evaluate mixing performance.

The model considers four basic cases with different agitator speeds to flow pattern sensitivity. The four cases consist of four different tank levels, $12,8.5,7$, and 4.5 inches, corresponding to fluid volumes of $38,21,19$, and 10 liters. Detailed operating conditions for the model are provided in Table 1.

Computational domains and meshes for the mixing tank filled to a 12-inch liquid level are presented in Figs. 5 and 6 . Finer nonuniform and hybrid meshes were used in the corner zones and joint sections near the agitator blade where flow direction changes and flow splits might occur. From a nodalization study, an optimum number of about 300,000 nodes was established for the final analysis of the three-dimensional model. As shown in 
the Fig. 6 , very fine nodes, less than 0.1 inch long, were used near the agitator hub and blade regions to capture the high velocity gradient.

The flow for the entire computational domain is assumed to be turbulent to give a reasonable representation of the liquid jet leaving the blade region. All converged solutions were achieved using the segregated and iterative solution technique.

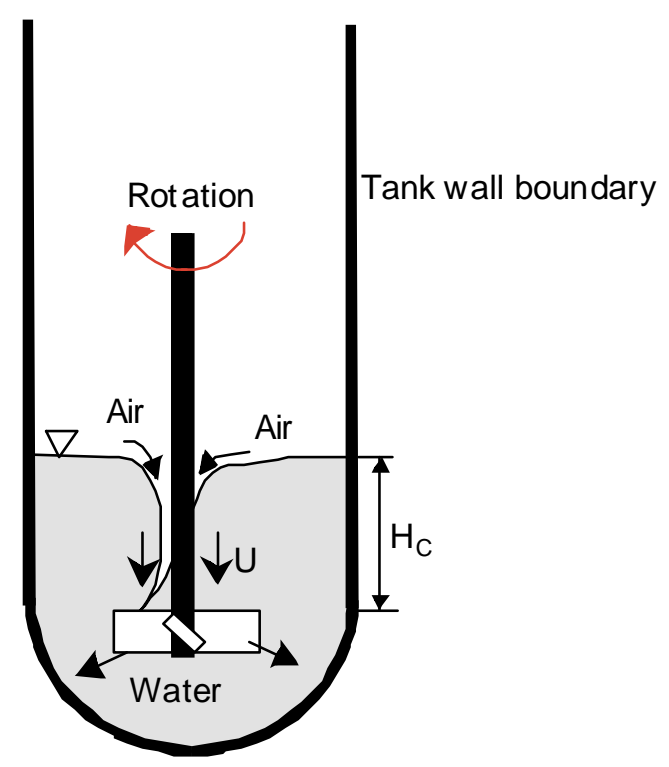

Figure 4. Air pull-through entrainment due to a large axial convective downward flow induced by high rotational speed of pitched agitator blade submerged in a low tank level 
Page: $\quad 10$ of 56

Table 1..Operating Conditions used for the modeling and analysis

\begin{tabular}{|c|c|}
\hline Operational Parameters & Operating Conditions \\
\hline $\begin{array}{c}\text { Mixing tank dimensions and agitator } \\
\text { geometry }\end{array}$ & See Figs. 1 and 2 \\
\hline $\begin{array}{c}\text { Tank liquid level from the tank bottom } \\
\text { (inches) }\end{array}$ & 12 (Reference case), 8.5, 7, 4.5 \\
\hline Blade elevation (inches) & 1 (above tank bottom) \\
\hline Rotational speed of agitator (rpm) & $300,400,500$ (Reference case), \\
600,700
\end{tabular}

Table 2. Flow conditions driven by the agitator shown in Fig. 1.

\begin{tabular}{|c|c|c|}
\hline $\begin{array}{c}\text { Pump Speed } \\
(\mathrm{rpm})\end{array}$ & $\begin{array}{c}\text { Agitator Reynolds number } \\
\left(\mathrm{Re}_{\mathrm{a}}\right)^{*}\end{array}$ & $\begin{array}{c}\text { Flow regime stirred } \\
\text { by agitator }\end{array}$ \\
\hline 300 & $1.827 \times 10^{4}$ & \multirow{2}{*}{$\begin{array}{c}\text { Turbulent flow } \\
\text { conditions } \\
\left(R e_{a}>10,000\right)\end{array}$} \\
\cline { 1 - 2 } & $2.436 \times 10^{4}$ & \\
\hline 500 & $3.045 \times 10^{4}$ & \\
\hline 600 & $3.654 \times 10^{4}$ & \\
\cline { 1 - 2 } & $4.263 \times 10^{4}$ & \\
\hline
\end{tabular}

Note: ${ }^{*} R e_{a}=\left(\frac{\rho_{f} N D_{B}^{2}}{\mu_{f}}\right)$, where $\mathrm{N}$ is the revolution of agitator per unit time. 


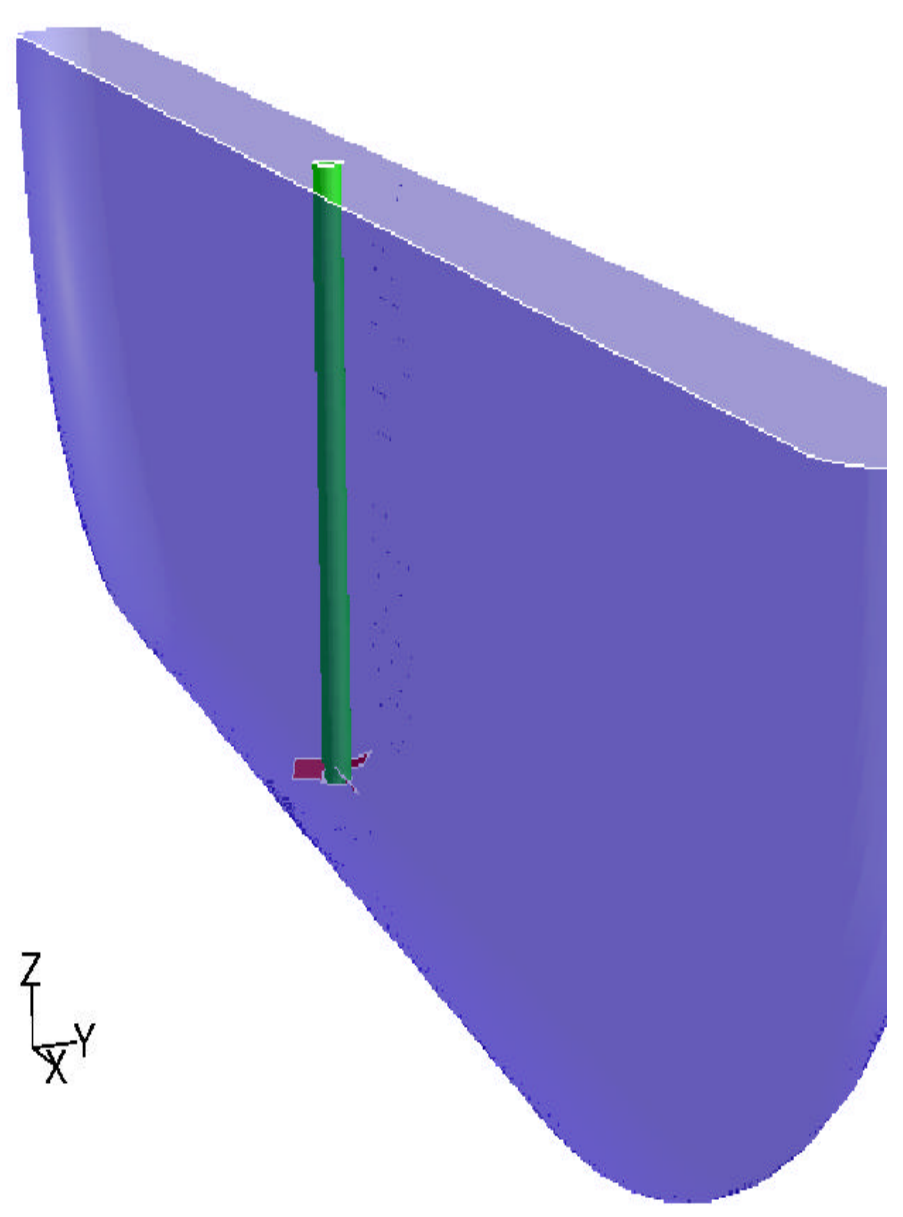

Figure 5. Geometrical configurations for 45-deg pitched three-blade agitator submerged in a flat tank as modeled for the present analysis. 
Page: $\quad 12$ of 56

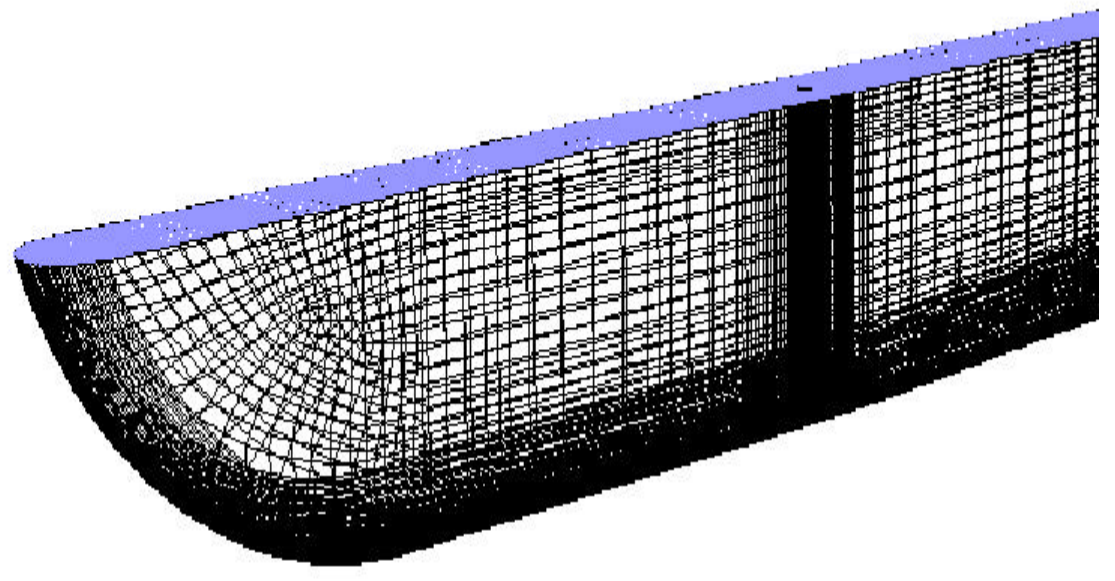<smiles>[Y]C([3H])[3H]</smiles>

Figure 6. Three-dimensional unstructured hybrid meshes for the flat tank with 45-deg pitched three-blade agitator as modeled for the present analysis. 


\section{Results and Discussions}

Computational results were evaluated based on several criteria. Critical velocity was used as a criterion to determine whether a given flow condition would result in the suspension of particles deposited on the tank bottom during precipitation. To ensure conservatism, a shear stress criterion was also applied for the scouring of particles from the bottom surface because of the interfacial drag at the solid surface. These criteria are summarized for the various tank levels in Table 3.

Tank liquid level was also a significant parameter. As the tank liquid level becomes lower, vortex formation can result in air being drawn into the blade zone of agitator as shown in Fig. 4. This can cause degradation of mixing capability in the tank. Therefore, a minimum liquid level must be estimated to avoid entraining air into the bulk fluid zone. Using the empirical correlation for air entrainment through the vortex formation, equation (7), the liquid level required to avoid air pull-through was estimated as function of agitator speed as shown in Table 4.

Four tank levels were evaluated to estimate the sensitivity of flow patterns to the rotational speed of the agitator. Steady-state calculations of the flow fields at various rotational speeds were performed for each tank level. A hybrid meshing approach employing hexahedral, pyramid-shaped, and tetrahedral elements in the computational domain was used. Mixing was simulated numerically using multiple reference frames.

The flow pattern discharged by an agitator depends on both the fluid mass inventory of tank and the agitator rotational speed. The distance through which a fluid element can travel to a remote area is proportional to the rate of kinetic energy dissipation of the fluid element at the point of discharge from an agitator blade [8]. For geometrically similar flow configurations, it can be shown by dimensional analysis that $\mathrm{P} / \mathrm{V}$, the time rate of fluid energy dissipation per unit volume, is governed by the well-established relationship,

$$
\frac{P}{V}=\left(\frac{A \rho_{f} U^{3}}{2 V}\right) \phi_{1}=\left(\frac{\rho_{f} U^{3}}{L_{c}}\right) \phi
$$

In equation (8), $L_{c}$ is a characteristic length associated with flow pattern and $\phi$ is a function of Reynolds number, which is related to the type of flow geometry involved and the particular characteristic velocity employed. The characteristic velocity could be the average velocity $U$ or the root-mean-squared (rms) fluctuating velocity $u$ ' at a certain position [9].

For all the present flow conditions, the agitator Reynolds number was found to be larger than $10^{4}$ as shown in Table 2. The agitator Reynolds number was based on the diameter of agitator and rotational speed as defined in the literature [12], that is,

$$
R e_{a}=\left(\frac{\rho_{f} U_{a} D_{B}}{\mu_{f}}\right)
$$

In equation (9) the agitator speed $U_{a}$ is used as the product of rotational speed $(M)$ and diameter $\left(D_{B}\right)$ of the agitator. This is twice the blade tip speed, and while it does not represent a physical speed within the tank, it is a typical parameter used in the mixing literature. 
Date: $\quad 07 / 24 / 02$

Page: $\quad 14$ of 56

The maximum fluid velocity discharged by the agitator is found to be proportional to the rotational speed of the agitator as expected. Figure 7 shows the results for six different rotational speeds from $150 \mathrm{rpm}$ to $700 \mathrm{rpm}$. The average local velocity available to pick up solid particles deposited near the corner of the tank is also proportional to the agitator speed, $N D_{B}$. The results for various liquid levels are presented in Fig. 8.

Using well established power correlations for fully turbulent flow stirred by an agitator [9, $10,11]$, it can be shown that, for fully-turbulent conditions as shown in Table 2, the power dissipation per unit volume is independent of the agitator Reynolds number, $R e_{a}$. It is given by the equation,

$$
\left(\frac{P}{V}\right)=\left(\frac{C^{\prime} \rho_{f} N^{3} D_{B}{ }^{5}}{D_{T}^{2} H}\right)
$$

In equation (10), $D_{T}$ and $H$ are the diameter and liquid height of an agitator tank. The right-hand side of equation (10) can be equated to the right-hand side of equation (8). Since $C^{\prime}$ in equation (10) is constant for turbulent flow, the parameter $\phi$ should be constant. Thus, when the agitator diameter $D_{B}$ is used for the characteristic length $L_{C}$, the resulting equation becomes

$$
U=C \frac{N D_{B}^{2}}{\left(D_{T}{ }^{2} H\right)^{1 / 3}}=C \theta
$$

In equation (11), all the constant terms have been lumped into C. From equation (11), the average velocity outside the agitator discharge zone was found to be proportional to the rotational speed of the agitator and its diameter squared, and inversely proportional to the cube root of the tank fluid volume. The current results were consistent with this relationship over a wide range of agitator speeds and tank levels, as shown in Fig. 9. The constant $C$ was found to be about 0.54 from a least squares fit with a maximum $20 \%$ uncertainty. The results are also consistent with literature data [11]. It is noted that this velocity correlation may have broad applicability, and maintaining a constant value of the parameter $\theta$ may be a reasonable scale-up criterion in many instances such as smallscale experimental tests and designs.

Five different rotational speeds were used for the agitator: $300,400,500,600$, and 700 rpm for each of the four different tank liquid levels as shown in Table 1. In previous work [3], applications of the two-equation $\kappa-\varepsilon$ turbulence model to the sudden change of flow direction were benchmarked against the test data with reasonable accuracy. A computational domain of about 300,000 nodes was found to give acceptable results. Typical running time for each modeling calculation was about 8 hours on an $866 \mathrm{MHz}$ Pentium III processor with 1GB of memory.

A series of calculations for the 12-inch liquid level were performed to estimate actual flow patterns representative of a 38-liter fluid volume in the HB-line tank with the agitator rotating in a clockwise direction as viewed from above. The results for the $300 \mathrm{rpm}$ case showed that both sides of the agitator had the least active flow zones. The fluid around the rotational axis of the agitator moved downward toward the agitator. It exited the agitator region divided into two main directions along the bottom surface of the tank as shown in Fig. 10. Velocity contour plots for 500 and 700 rpm are shown in Figs. 11 and 12 , respectively. The flow patterns did not change significantly as the agitator speed 
increased. Table 5 shows the quantitative results for the flow patterns at different agitator speeds. These values relate closely to the pick-up and suspension of particles deposited on the bottom surface of the tank. Figure 13 also shows the results of flow patterns inside the fluid domain for different locations and speeds.

Wall shear stress was also evaluated as a mixing criterion. It was assumed that particleparticle interactions and the effects of the particle presence on the continuous liquid phase were negligible for small particles ( 20 microns). The critical shear stress required to scour a 20-micron particle was found to be about $0.033 \mathrm{~Pa}$. Typical results for shear stress distributions over the range of speeds in the 12-inch tank level are shown in Fig. 14. The results showed that wall shear stress for agitator speeds higher than 500 rpm was larger than the critical shear stress for all the liquid levels considered here. Therefore, the critical velocity was used as the primary criterion to determine the agitator speed to achieve adequate solid mixing in the tank.

Pressure distributions around the agitator for the reference case (12-inch level and 500 rpm) are presented in Fig. 15. The results show that the region behind the agitator blade has negative gauge pressure. This makes the fluid move into the agitator region and flow in the downward direction. Figure 16 presents flow patterns on the center plane of the agitator in terms of velocity vectors corresponding to the pressure distribution shown in Fig. 15. Overall flow patterns for three-dimensional flow domain are also shown in Fig. 17. When looking down from the top of the tank, detailed flow patterns near the agitator and its rotational axis are shown in Fig. 18. When the agitator rotates in a clockwise direction, fluid near the rotational axis of the agitator moves downward, and the maximum flow velocity occurs near the tip of the agitator blade. Figure 19 shows flow patterns for the two-dimensional fluid domain below the agitator. It is noted that the downward flow at the downstream side of the agitator is mainly split into two horizontal directions toward the tank corners along the bottom surface of the tank. Figure 20 shows velocity distributions for various agitator speeds at a local position 23.5 inches distant from the agitator shaft.

The turbulent flow fields that develop in an agitated tank are generated by the rotational motion of the agitator. Agitator flow phenomena include high-speed discharge flow, blade boundary layers, blade wake regions, boundary layer separation regions, and trailing vortex systems. An understanding of the velocity flow fields is a prerequisite to understanding mixing and key physical parameters such as shear stress, flow fluctuations, and vorticity fields. The wall shear stress distributions on the agitator blades, as shown in Fig. 21, provide a direct indication of flow field development resulting from the no-slip condition at the blade wall. The fluid particles near the blade wall are accelerated by an imbalance of shear forces.

$$
\nabla \bullet \tau=\mu \nabla^{2} \vec{v}=-\mu \nabla \times(\nabla \times \vec{v})=-\mu \nabla \times \omega
$$

As shown in equation (12), an unbalanced shear stress can only exist when the vorticity is nonzero. Thus, the existence of vorticity means that a fluid particle is subjected to net viscous forces. Figure 22 shows flow patterns, velocity magnitudes, turbulence intensity, and vorticity fields. The results show that the strongest discharge flow fields occur at the downstream side of blade, and flow fluctuations and vortex motion originate from the tip of the blade. It is noted that vortex flow dissipates from its point of origin at blade tip to the downstream region as shown in Fig. 22. 
Figure 23 shows pressure, velocity, and turbulence intensity distributions for 500 rpm operation at the vertical plane crossing the agitator center for an 8.5-inch liquid level. These results are consistent with the literature. Horizontal velocity contour plots at several different elevations are presented in Fig. 24. The figure shows the fluid zones with a velocity higher than the minimum critical velocity for 20 -micron solids and a specific gravity of 2.5 .

Vertical flow patterns at several different distances from the agitator are shown in Fig. 25. Corresponding turbulence intensities are presented in Fig. 26. Vertical snapshots of the fluid velocity distributions at different distances from the agitator are shown in Figs. 27 and 28 for agitator speeds from 300 to $700 \mathrm{rpm}$. Figure 29 shows the fluid domain having velocities higher than the critical velocity for a $600 \mathrm{rpm}$ agitator speed and an 8.5inch tank level. The critical velocity for this case is $0.225 \mathrm{ft} / \mathrm{sec}$ for a 20 -micron solid particle as shown in Table 3.

Figure 30 shows the sensitivity results for fluid velocity magnitude at a given position as the agitator speed increases from 300 to $700 \mathrm{rpm}$. Velocity distributions along the vertical direction from the tank bottom at various horizontal distances from the agitator are presented for the 700 rpm case in Fig. 31.

Figure 32 shows velocity contour plots at the vertical plane crossing the agitator centerline for various different speeds and a 7-inch liquid level. Velocity distributions for the different agitator speeds at a horizontal distance of 23.5 inches from the agitator are shown in Fig. 33. For this case, a rotational speed higher than $500 \mathrm{rpm}$ is required for acceptable solid mixing.

Sensitivity studies of local flow fields were performed for rotational speeds from 400 to $600 \mathrm{rpm}$ and a 4.5-inch tank level, the lowest of the four tank levels evaluated. The results showed that the $500 \mathrm{rpm}$ agitator speed generated adequate flow fields to mix the solids without entraining air for the 4.5-inch liquid level as shown in Fig. 34 . Figure 35 shows graphical snapshots for two different speeds, 400 and $500 \mathrm{rpm}$.

It is important to evaluate the impact of tank liquid level on the flow fields developed for a given agitating speed. Local velocity distributions for various liquid levels with a $500 \mathrm{rpm}$ agitator speed at a position 23.5 inches away from the agitator are presented in Fig. 36 . The results for $700 \mathrm{rpm}$ are shown in Fig. 37. The results show a tank with a lower liquid level has more agitation for a given agitator speed, assuming that flow velocity is the primary indicator of mixing performance. These results are consistent with Fig. 9 in terms of non-dimensional parameter $\theta$, which is related to the ratio of power dissipation $(P)$ to tank fluid volume $(V)$.

All the above analyses were made with a steady-state model assuming that the top liquid surface remains stationary and at atmospheric pressure. The minimum liquid level to prevent air entrainment into the blade zones and thereby to avoid degrading the mixing performance was estimated by using the empirical correlation in equation (6). As a scoping analysis, a transient model incorporating the motion of top liquid surface was developed, but it required a large amount of computational time to capture the transient behavior of the free surface. One of the early transient snapshots for the free surface motion is shown in Fig. 38 indicating that the liquid level near the axis of the agitator becomes lower compared to the other region as transient time goes on. 
All the modeling results for various tank levels and pump speeds are compared with critical velocities in Table 6 . As shown in the table, potential operating conditions shown in red italics satisfy critical criteria for acceptable mixing. Typical flow patterns for high and low tank levels and a fully-submerged are presented in Fig. 39.

The flow fields occurring under turbulent flow conditions can be based on the initial blade selection for the agitator. The flow phenomena generated by the agitator include convection flow resulting from the blade shape, blade boundary layers, and trailing vorticity. Figure 40 shows the primary flow components induced by the agitator when a pitched or flat blade agitator rotates clockwise.

Table 3. Critical velocities for various liquid levels to initiate the movement for 20-micron solid particles deposited at the bottom of flat tank

\begin{tabular}{|c|c|c|c|}
\hline \multirow{2}{*}{$\begin{array}{c}\text { Liquid Level } \\
\text { (inches) }\end{array}$} & \multicolumn{2}{|c|}{$\begin{array}{c}\text { Critical Velcity } \\
\left(U_{c}\right)\end{array}$} & $\begin{array}{c}\text { Critical Shear Stress } \\
\left(\tau_{w c}\right)\end{array}$ \\
\cline { 2 - 4 } & $(\mathrm{ft} / \mathrm{sec})$ & $(\mathrm{m} / \mathrm{sec})$ & 0.033 \\
\hline 4.5 & 0.211 & 0.064 & 0.033 \\
\hline 7.0 & 0.221 & 0.067 & 0.033 \\
\hline 8.5 & 0.225 & 0.069 & 0.033 \\
\hline 12.0 & 0.233 & 0.071 & \\
\hline
\end{tabular}

Table 4. Conservative estimation of liquid level for the inception of air pull-through

\begin{tabular}{|c|c|c|}
\hline \multirow{2}{*}{$\begin{array}{c}\text { Pump Speed } \\
\text { (rpm) }\end{array}$} & \multicolumn{2}{|c|}{$\begin{array}{c}\text { Minimum Liquid Level to Avoid Air Entrainment }\left(H_{c}\right) \\
\text { (inches) }\end{array}$} \\
\cline { 2 - 3 } & $\begin{array}{c}\text { Liquid height above the } \\
\text { agitator blade }\end{array}$ & $\begin{array}{c}\text { Liquid height above the } \\
\text { tank bottom }\end{array}$ \\
\hline 300 & 2.30 & 3.65 \\
\hline 400 & 2.55 & 3.90 \\
\hline 500 & 2.83 & 4.18 \\
\hline 600 & 3.13 & 4.48 \\
\hline 700 & 3.27 & 4.62 \\
\hline
\end{tabular}


Report: WSRC-TR-2002-00219

Date: $\quad 07 / 24 / 02$

Page: $\quad 18$ of 56
WESTINGHOUSE SAVANNAH RIVER COMPANY

AGITATOR MIXING ANALYSIS IN A HB-LINE FLAT TANK

Table 5. Average local velocity magnitudes for various agitator speeds at two different distances (18 inches and 23.5 inches) from the agitator submerged in 12-inch tank level

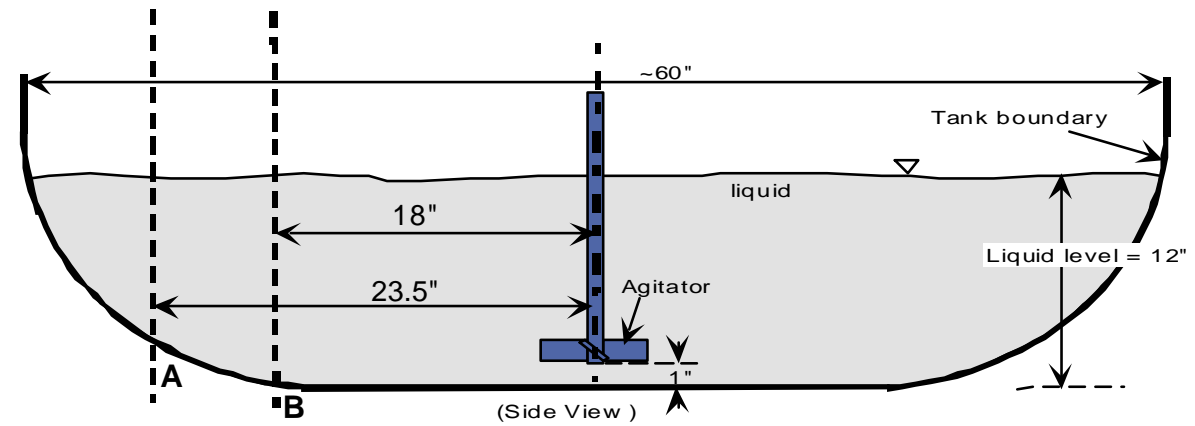

\begin{tabular}{|c|c|c|}
\hline $\begin{array}{c}\text { Pump Speed } \\
\text { (rpm) }\end{array}$ & $\begin{array}{c}\text { Average Local Velocity at } \\
\text { Location B (m/sec) }\end{array}$ & $\begin{array}{c}\text { Average Local Velocity at } \\
\text { Location A (m/sec) }\end{array}$ \\
\hline 300 & 0.0346 & 0.0320 \\
\hline 400 & 0.0474 & 0.0448 \\
\hline 500 & 0.0602 & 0.0578 \\
\hline 600 & 0.0731 & 0.0709 \\
\hline 700 & 0.0859 & 0.0839 \\
\hline
\end{tabular}

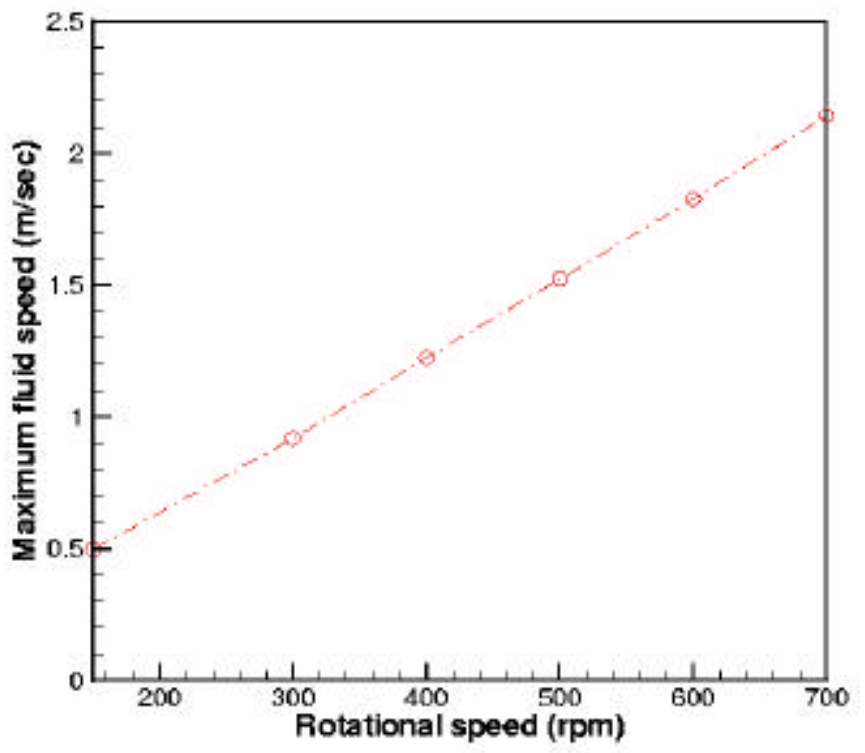


Figure 7. Maximum fluid velocity as function of the rotational speed of $45^{\circ}$ pitched agitator submerged in flat tank.
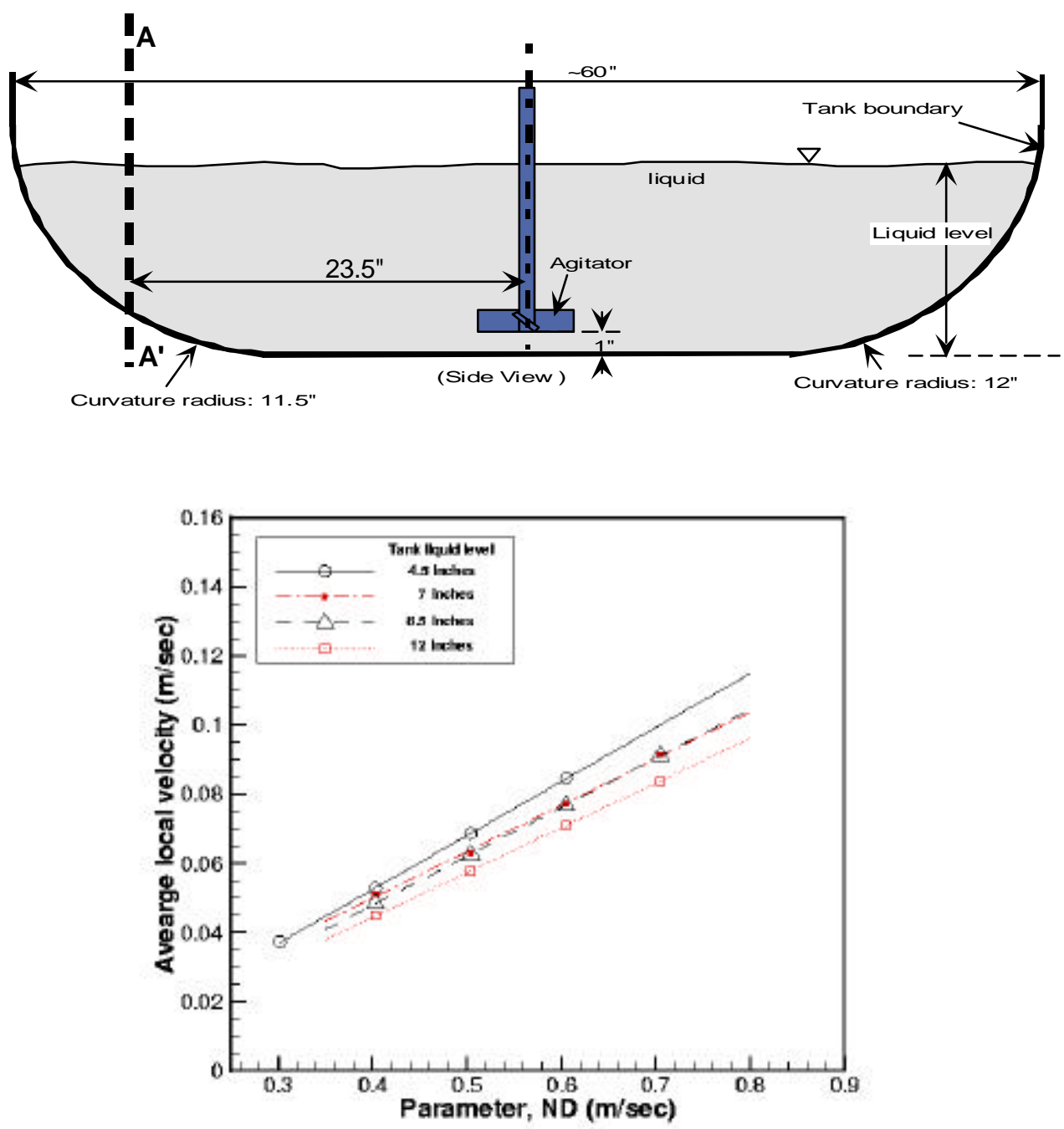

Figure 8. Average local velocity versus the product parameter of the rotational speed $(\mathrm{N})$ and blade diameter $\left(D_{B}\right)$ for three-blade $45^{\circ}$ pitched agitator submerged in flat tank. The average velocity was obtained by averaging local velocities along the line A-A'. 

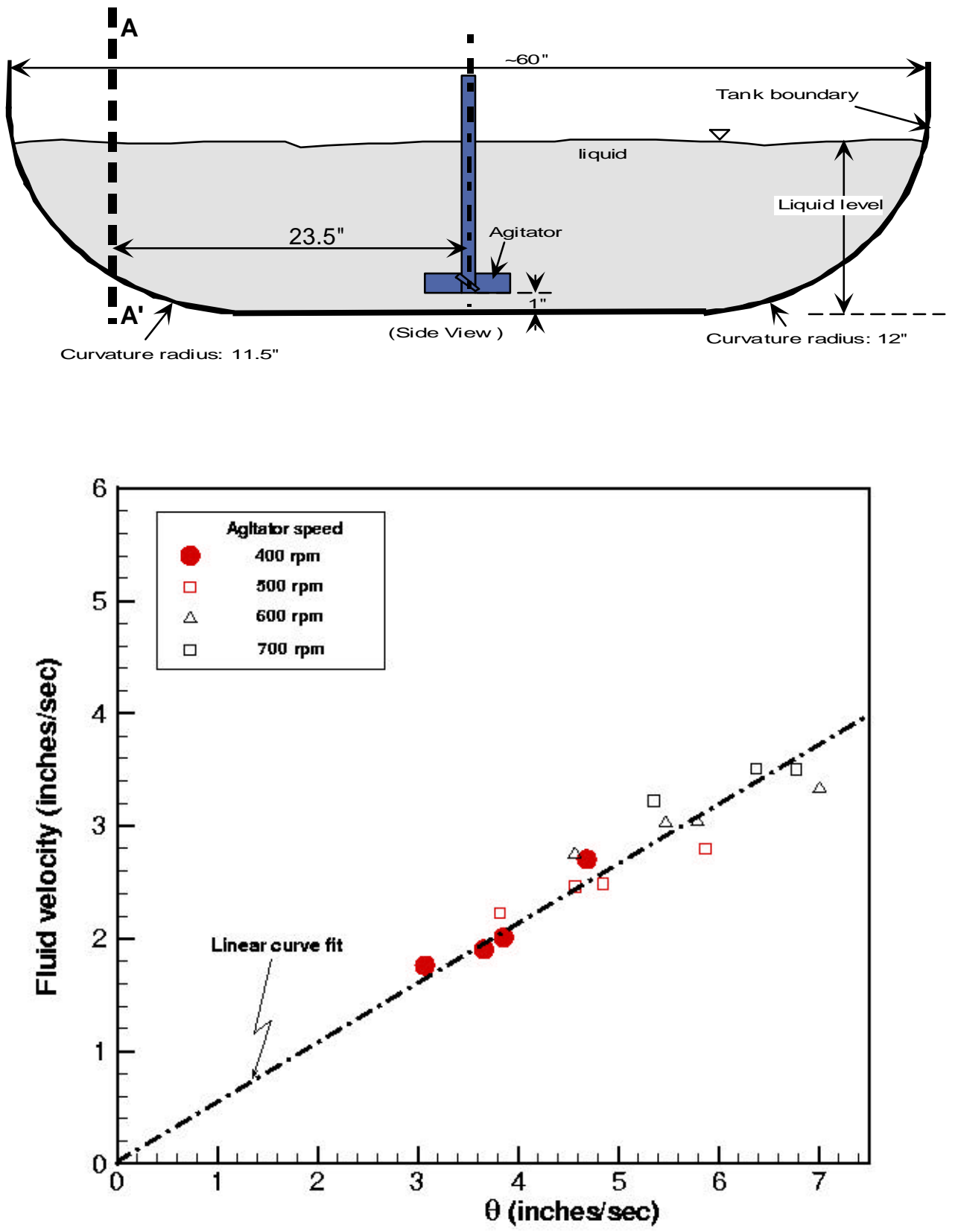

The parameter $\theta$ shown in the figure is defined as $\theta=\left(\frac{N D_{B}{ }^{2}}{\left(D_{T}{ }^{2} H\right)^{1 / 3}}\right)$.

Figure 9. Average local fluid velocity at the corner of tank bottom versus the parameter $(\theta)$ related to mixing performance (Slope of the linear fit is about 0.54.) The average velocity was obtained by averaging local velocities along the line A-A'. 

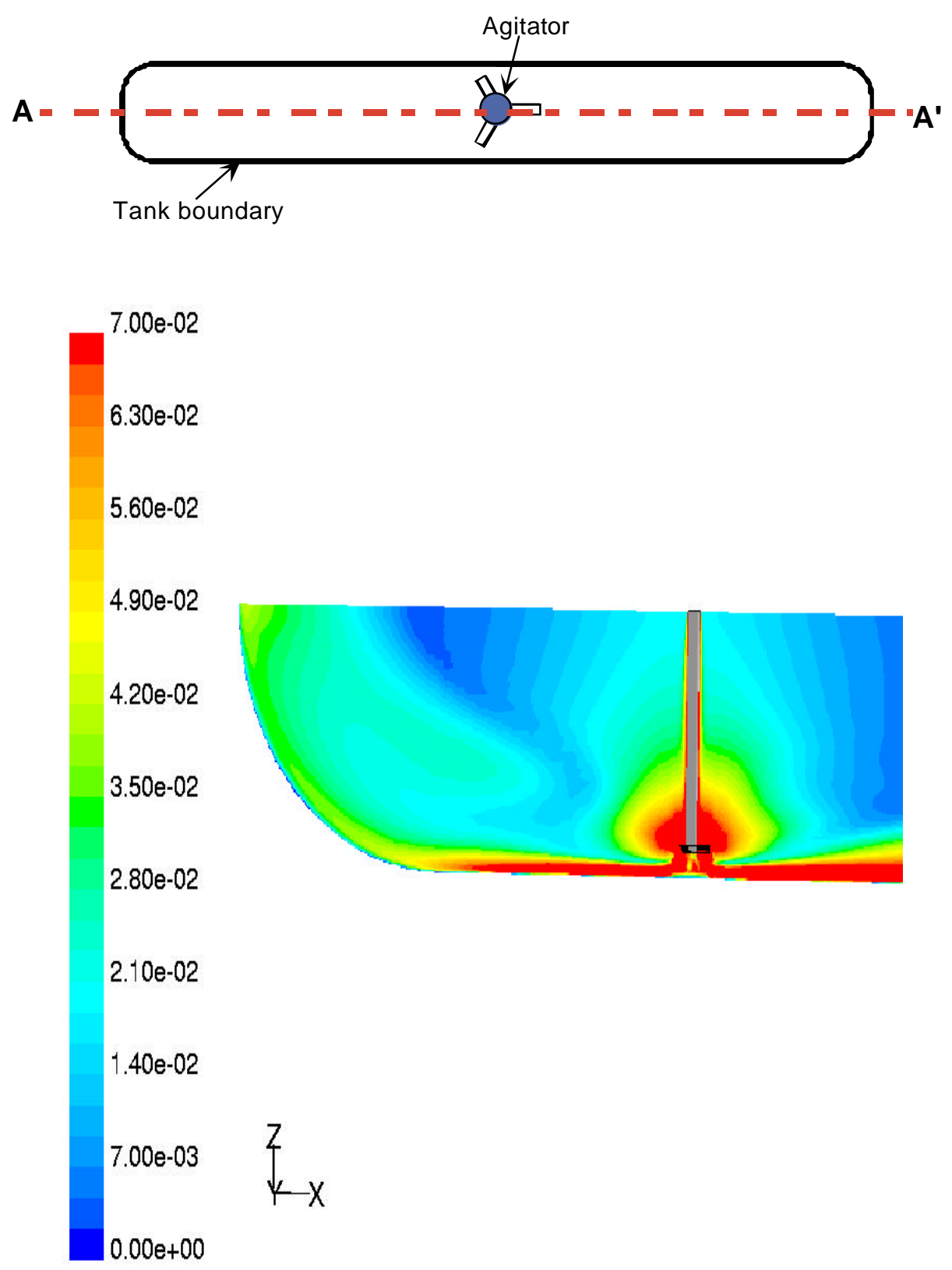

Contours of Velocity Magnitude $(\mathrm{m} / \mathrm{s})$

FLUENT 5

Figure 10. Velocity contour plot at the plane A-A' of the mixing tank with 300 rpm agitator speed and 12-inch liquid level 

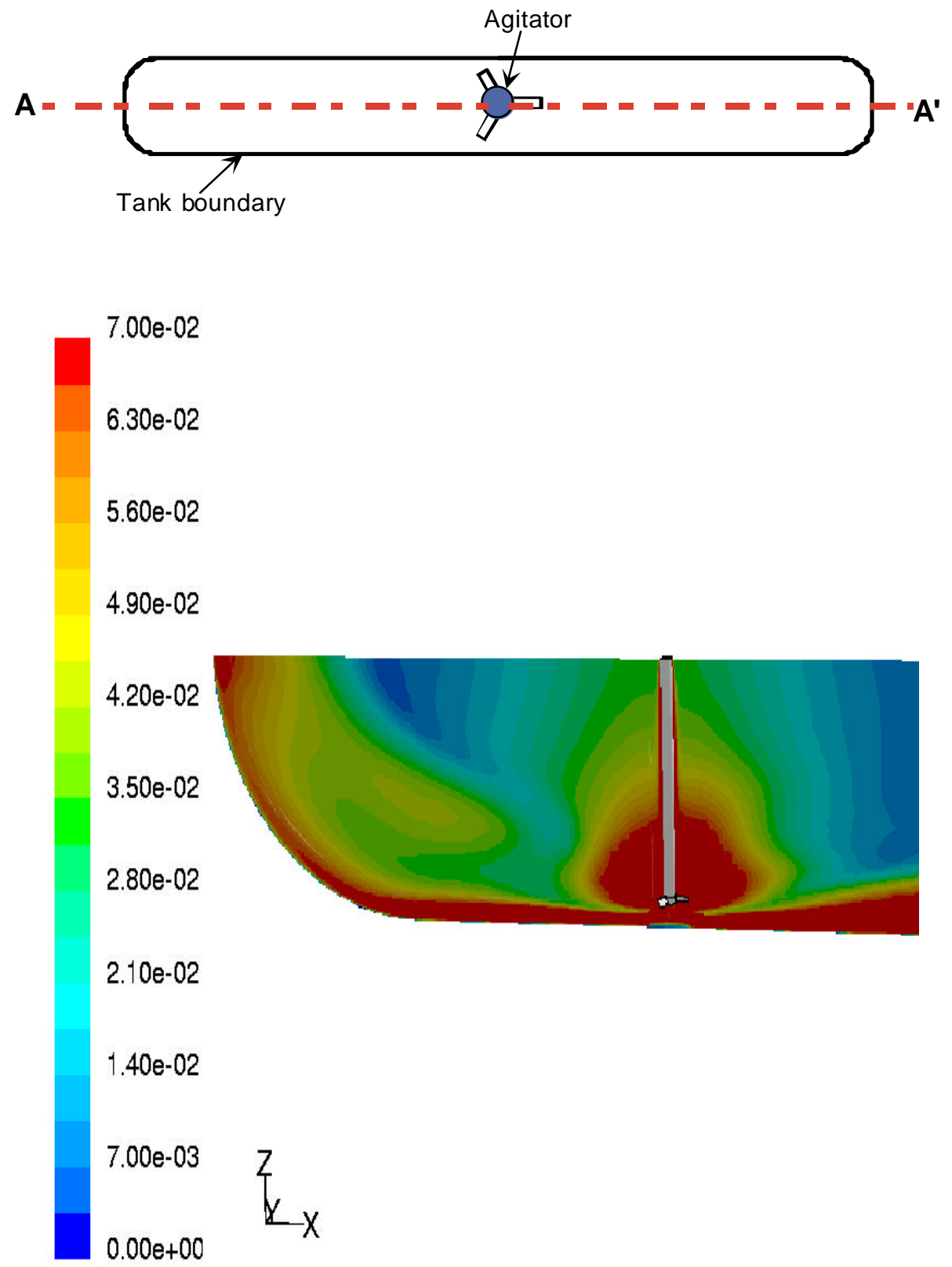

Contours of Velocity Magnitude (m/s)

\section{FLUENT 6.C}

Figure 11. Velocity contour plot at the plane A-A' of the mixing tank for the reference case shown in Table 1 (12-in tank level and 500 rpm agitator speed) 

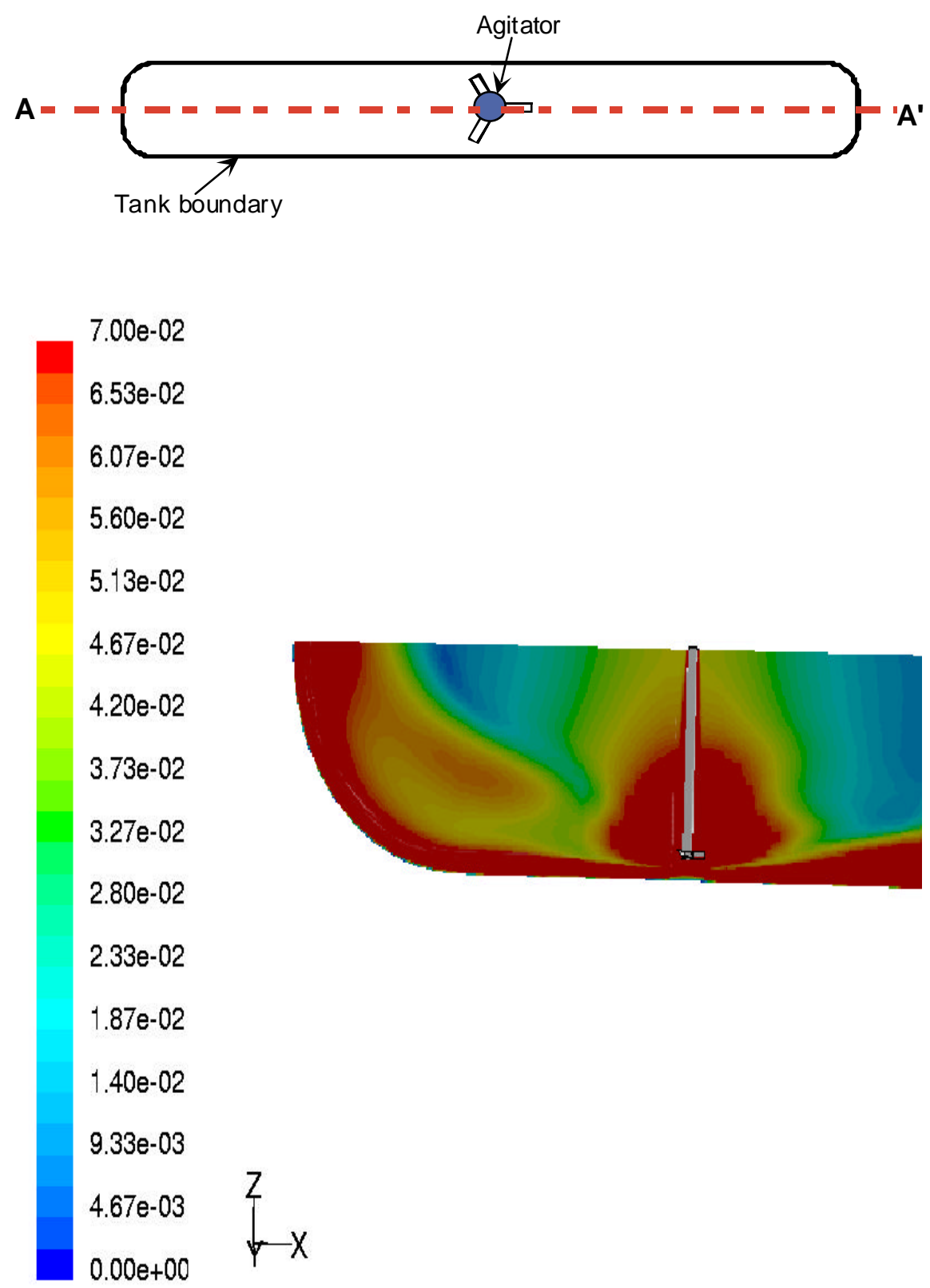

Contours of Velocity Magnitude (m/s)

FLUENT 6.C

Figure 12. Velocity contour plot at the plane A-A' of the mixing tank with 12 -inch liquid level and $700 \mathrm{rpm}$ agitator speed 

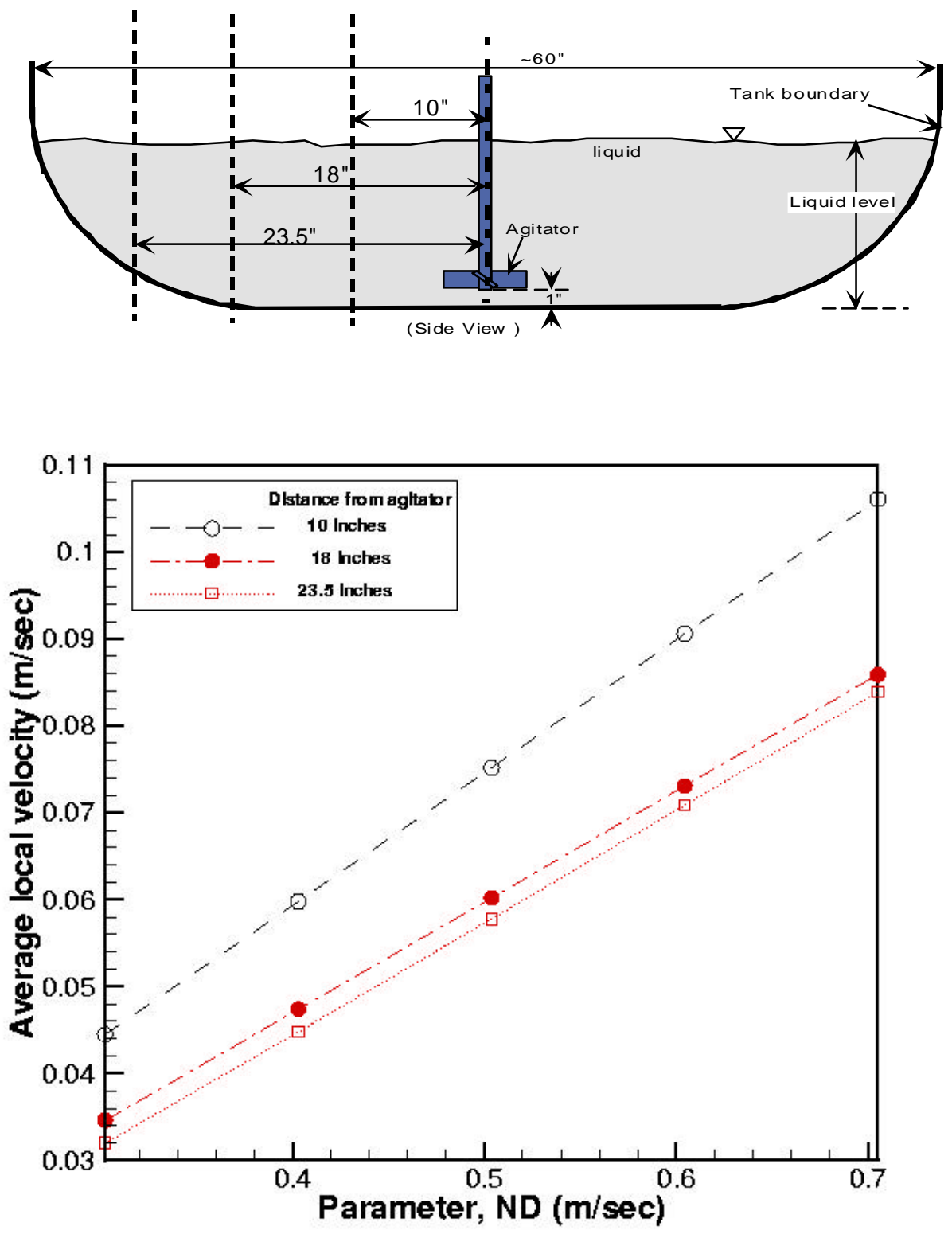

Figure 13. Average local velocity magnitude versus the parameter (ND) of the agitator speed for various distances from the agitator submerged in 12-inch tank level 

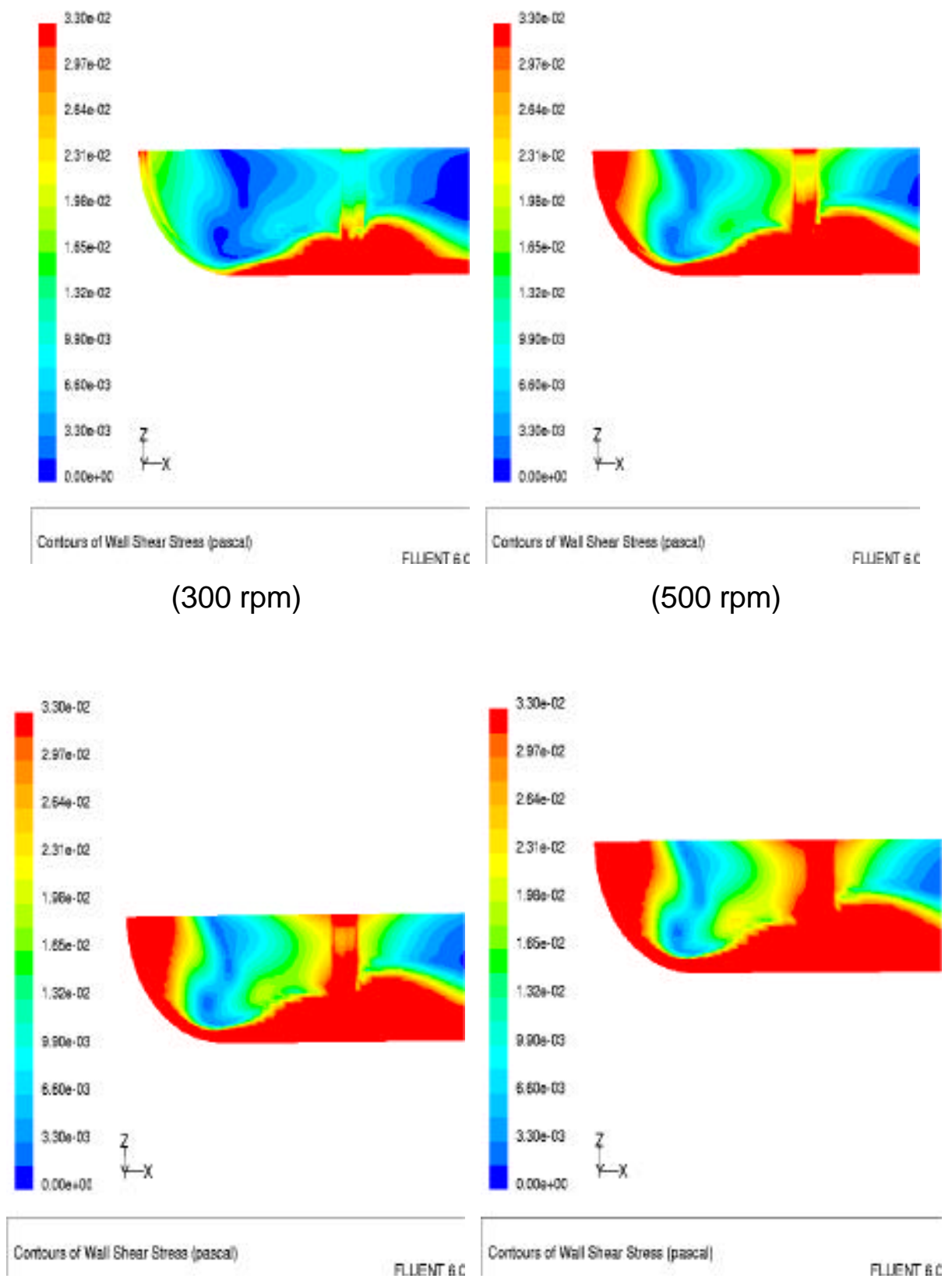

Contous of Wall Shear Streas (pacca)

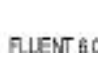

(600 rpm)

(700 rpm)

Figure 14. Shear stress contour plot at the wall surface of mixing tank for different speeds of the agitator submerged in 12-inch tank level showing the red zone to be larger than critical shear stress for 20 micron particle 

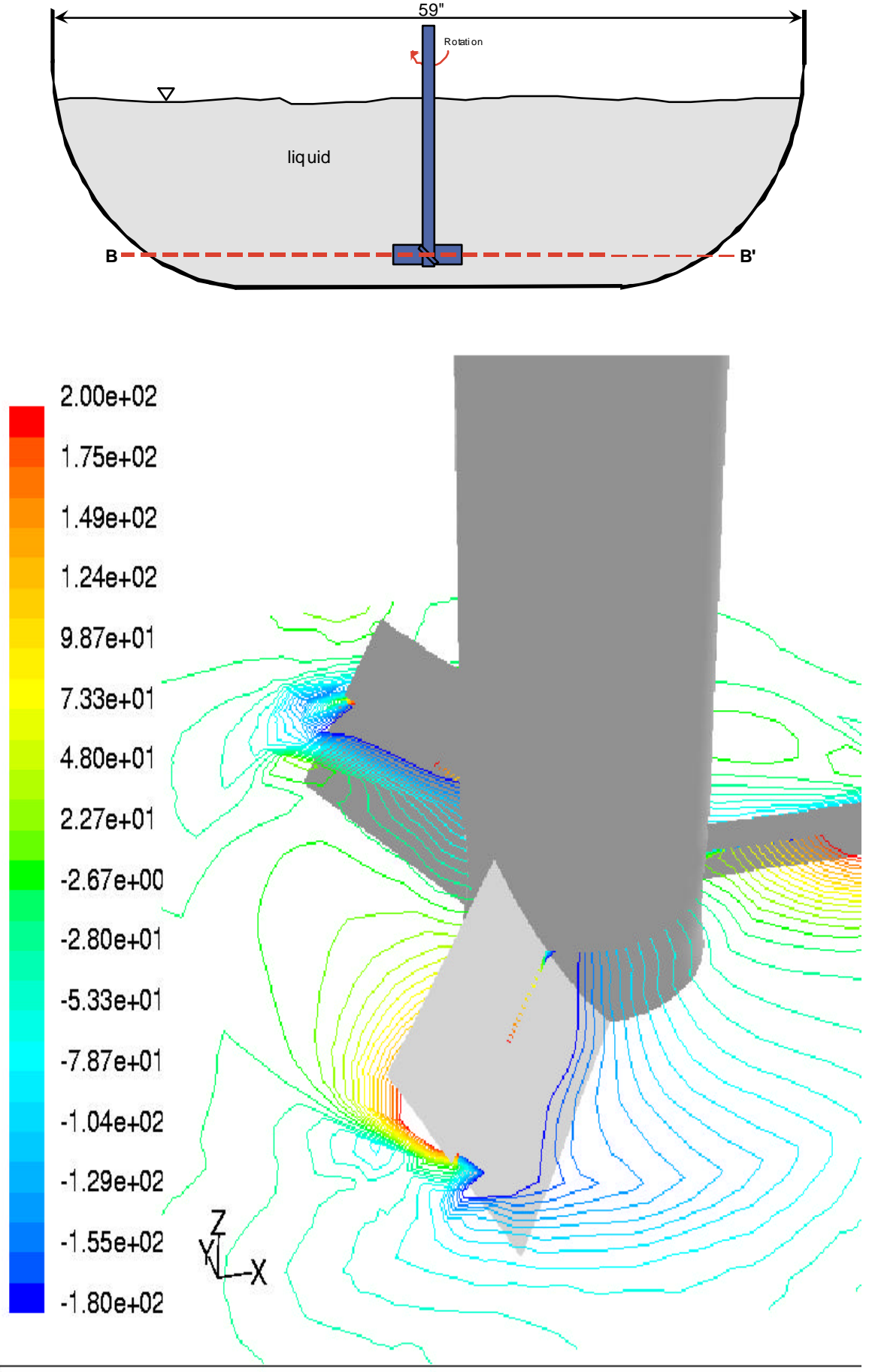

Contours of Static Pressure (pascal)

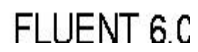

Figure 15. Pressure contour plot on the horizontal plane crossing the blade of the agitator (plane B-B') submerged in a flat tank for the reference case shown in Table 1 (12-in tank level and agitator speed = 500 rpm) 

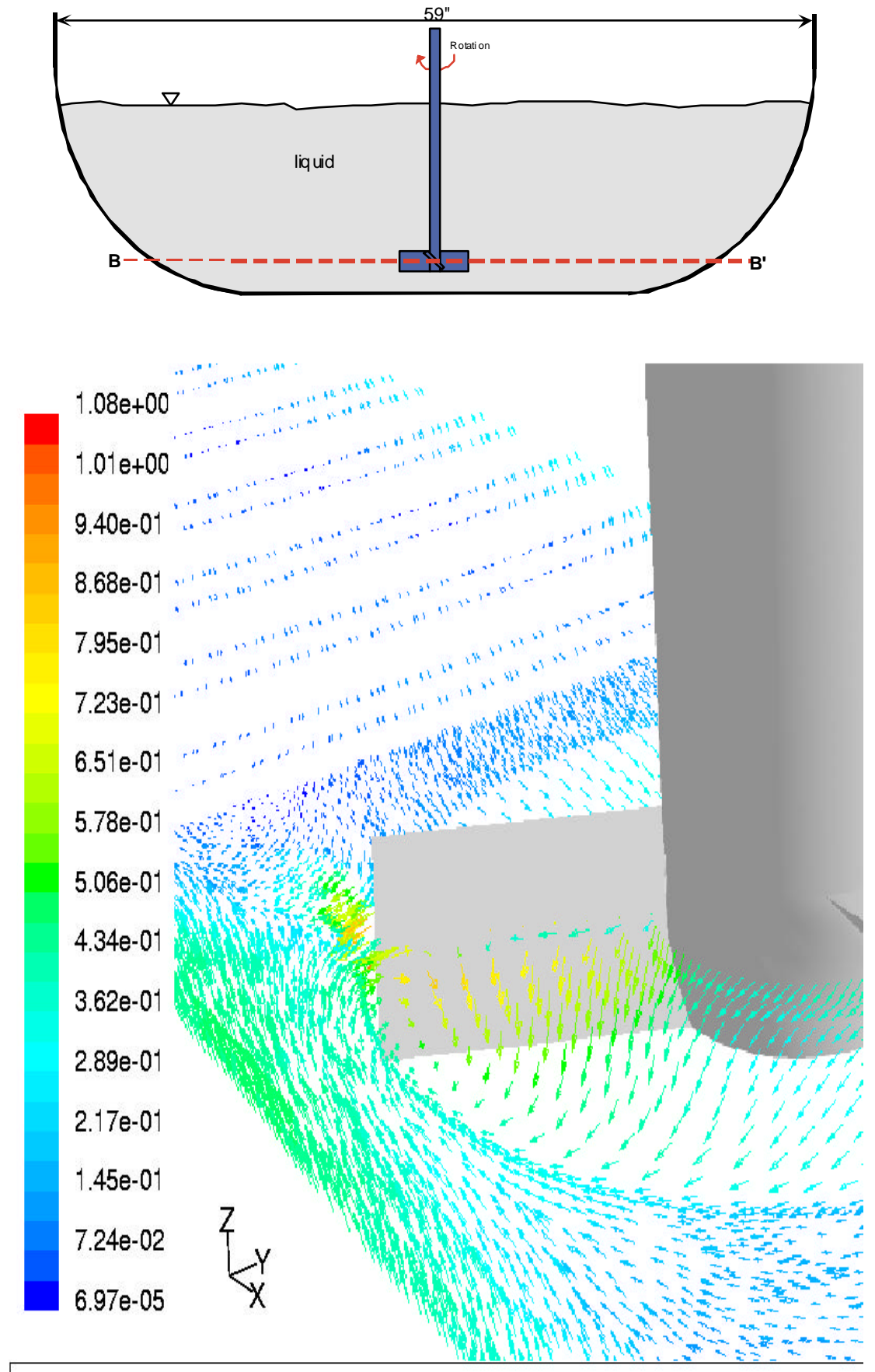

Velocity Vectors Colored By Velocity Magnitude ( $\mathrm{m} / \mathrm{s}$ )

FLUENT 6.C

Figure 16. Velocity vector plot on the horizontal plane crossing the blade for the reference case shown in Table 1 (12-in tank level and agitator speed = $500 \mathrm{rpm})$ 
Report: WSRC-TR-2002-00219

Date: $\quad 07 / 24 / 02$

Page: $\quad 28$ of 56

$7.00 \mathrm{e}-01$
$6.53 \mathrm{e}-01$
$6.07 \mathrm{e}-01$
$5.60 \mathrm{e}-01$
$5.13 \mathrm{e}-01$
$4.67 \mathrm{e}-01$
$4.20 \mathrm{e}-01$
$3.73 \mathrm{e}-01$
$3.27 \mathrm{e}-01$
$2.80 \mathrm{e}-01$
$2.33 \mathrm{e}-01$
$1.87 \mathrm{e}-01$
$1.40 \mathrm{e}-01$
$9.34 \mathrm{e}-02$
$4.67 \mathrm{e}-02$
$6.97 \mathrm{e}-05$

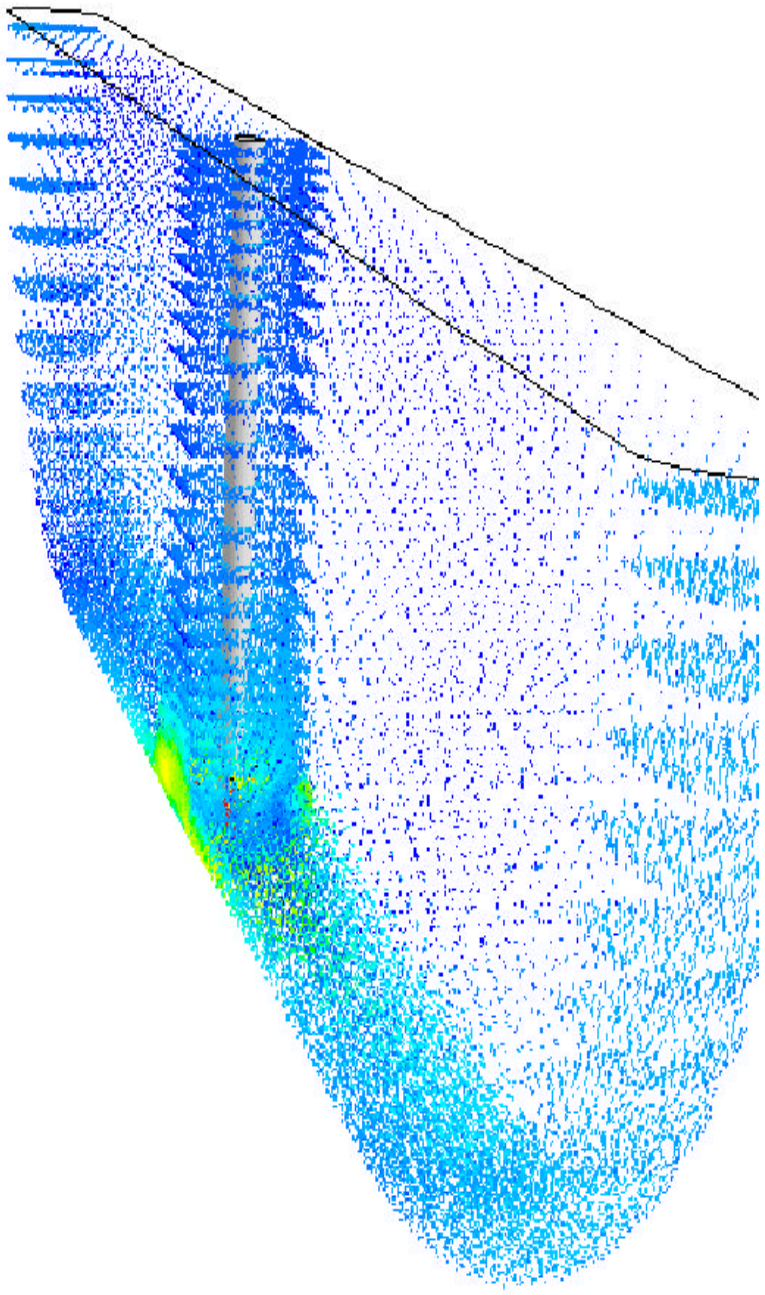

Velocity Vectors Colored By Velocity Magnitude $(\mathrm{m} / \mathrm{s})$

\section{FLUENT 6.C}

Figure 17. Velocity vector plot in three-dimensional fluid domain for the reference case shown in Table 1 (12-in tank level and agitator speed = 500 rpm) 


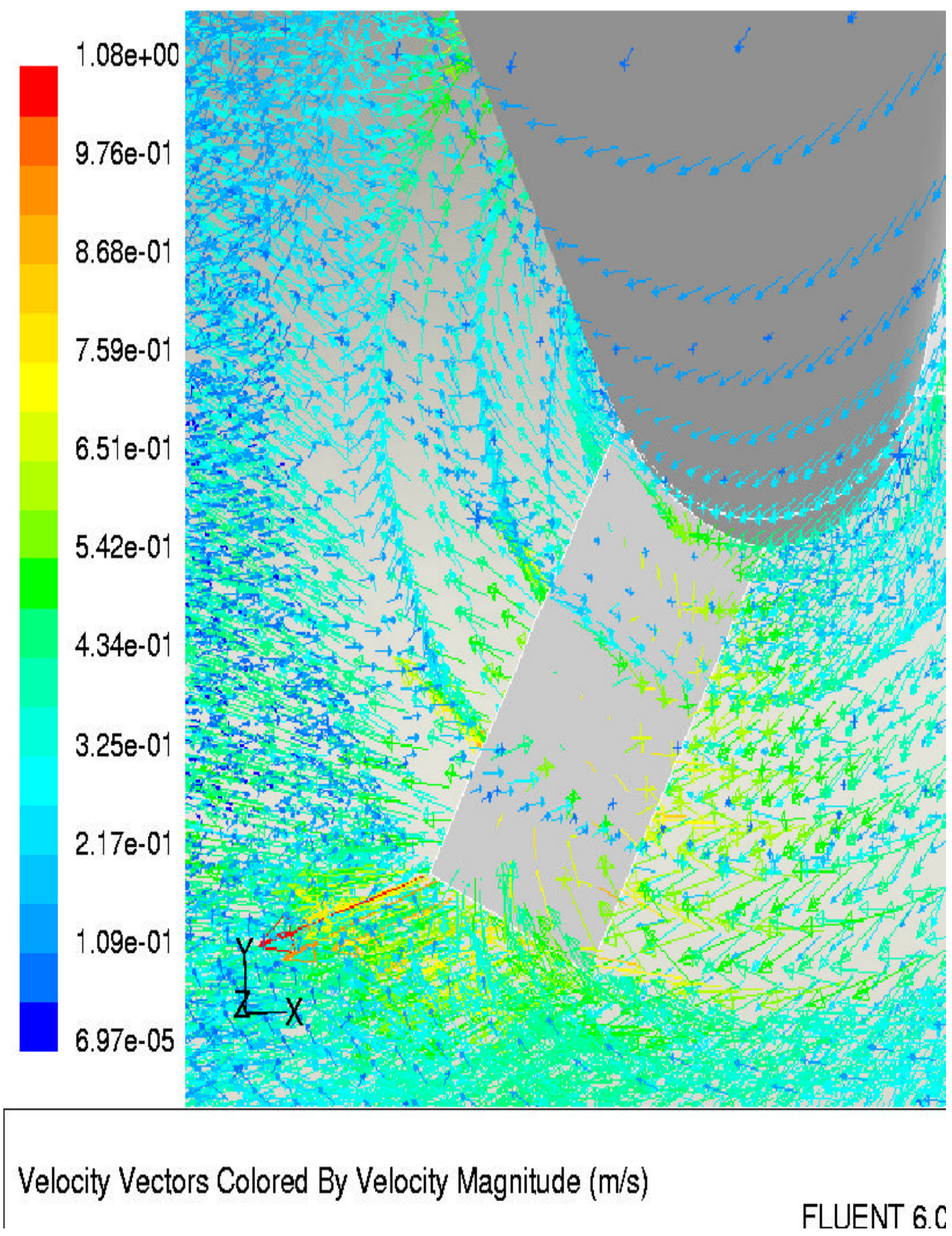

Figure 18. Three-dimensional velocity vector plot near the agitator blade from the top of the tank for the reference case shown in Table 1 (12-in tank level and agitator speed $=500 \mathrm{rpm}$ ) 

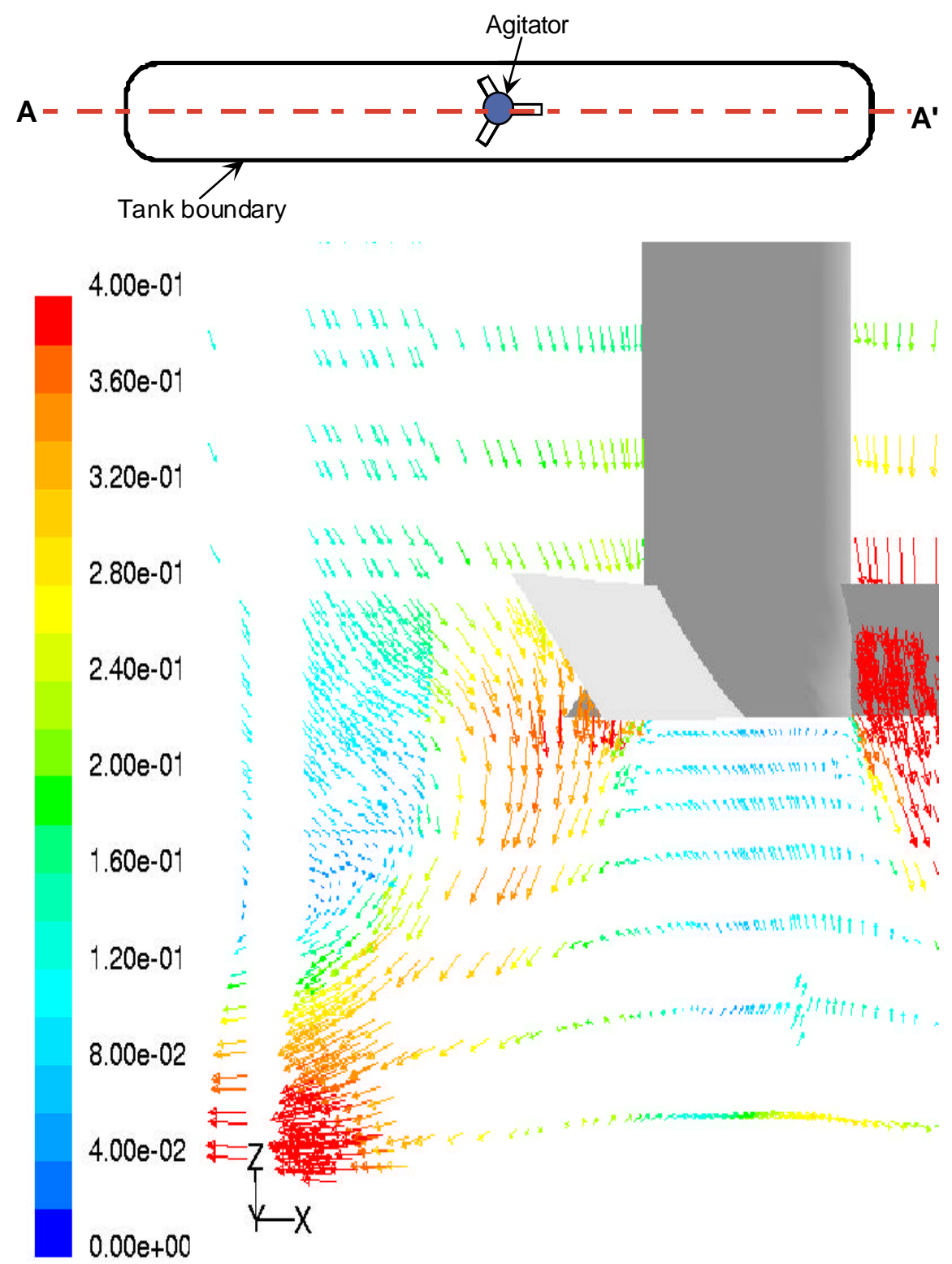

Velocity Vectors Colored By Velocity Magnitude $(\mathrm{m} / \mathrm{s})$

\section{FLUENT 6.C}

Figure 19. Velocity vector plot near the agitator blade at the mid-plane of the tank (plane A-A') for the reference case shown in Table 1 (12-in tank level and agitator speed $=500 \mathrm{rpm}$ ) 

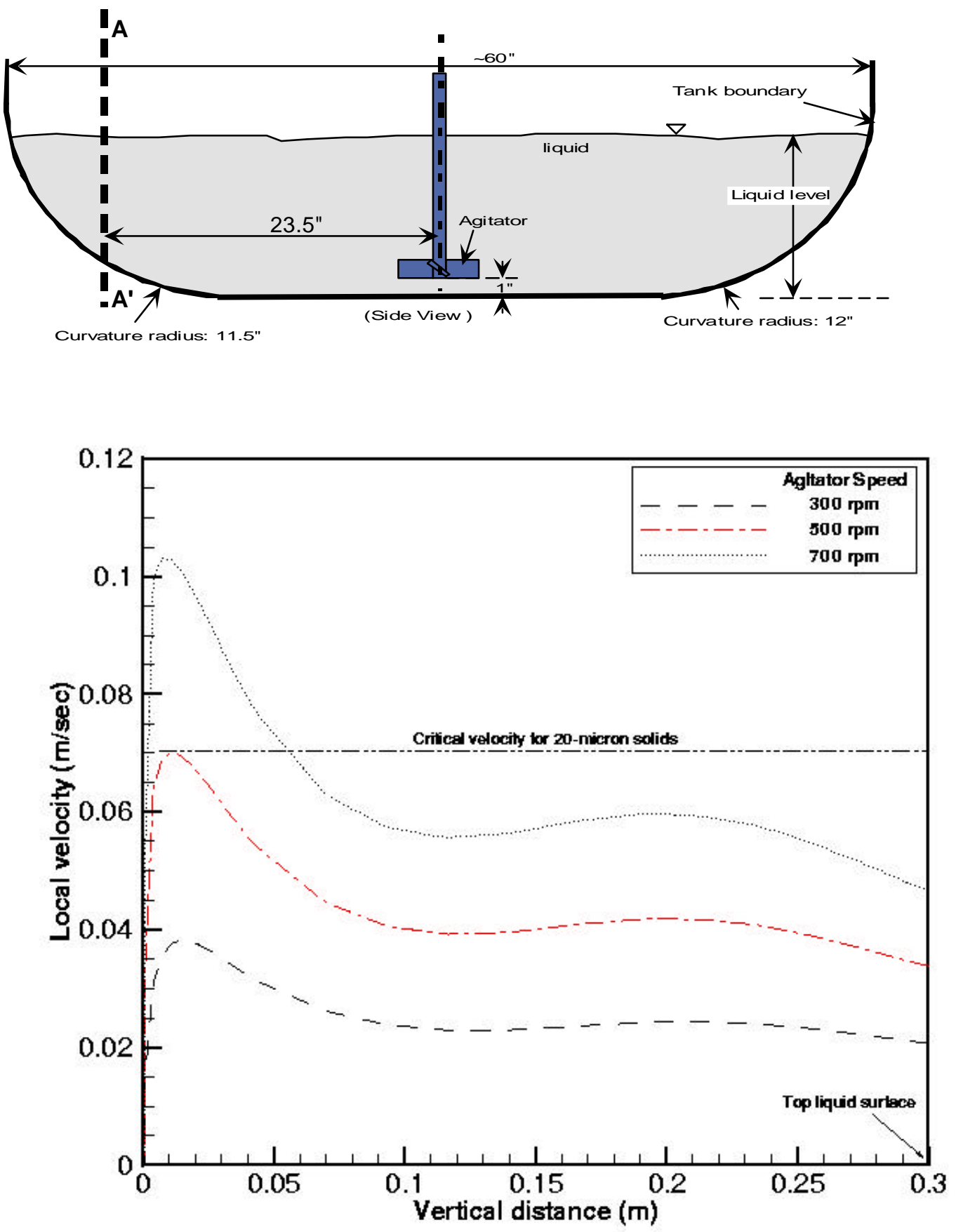

Figure 20. Velocity distributions at the vertical plane crossing the line A-A' for three different agitator speeds in the flat tank filled with 12-inch liquid level 

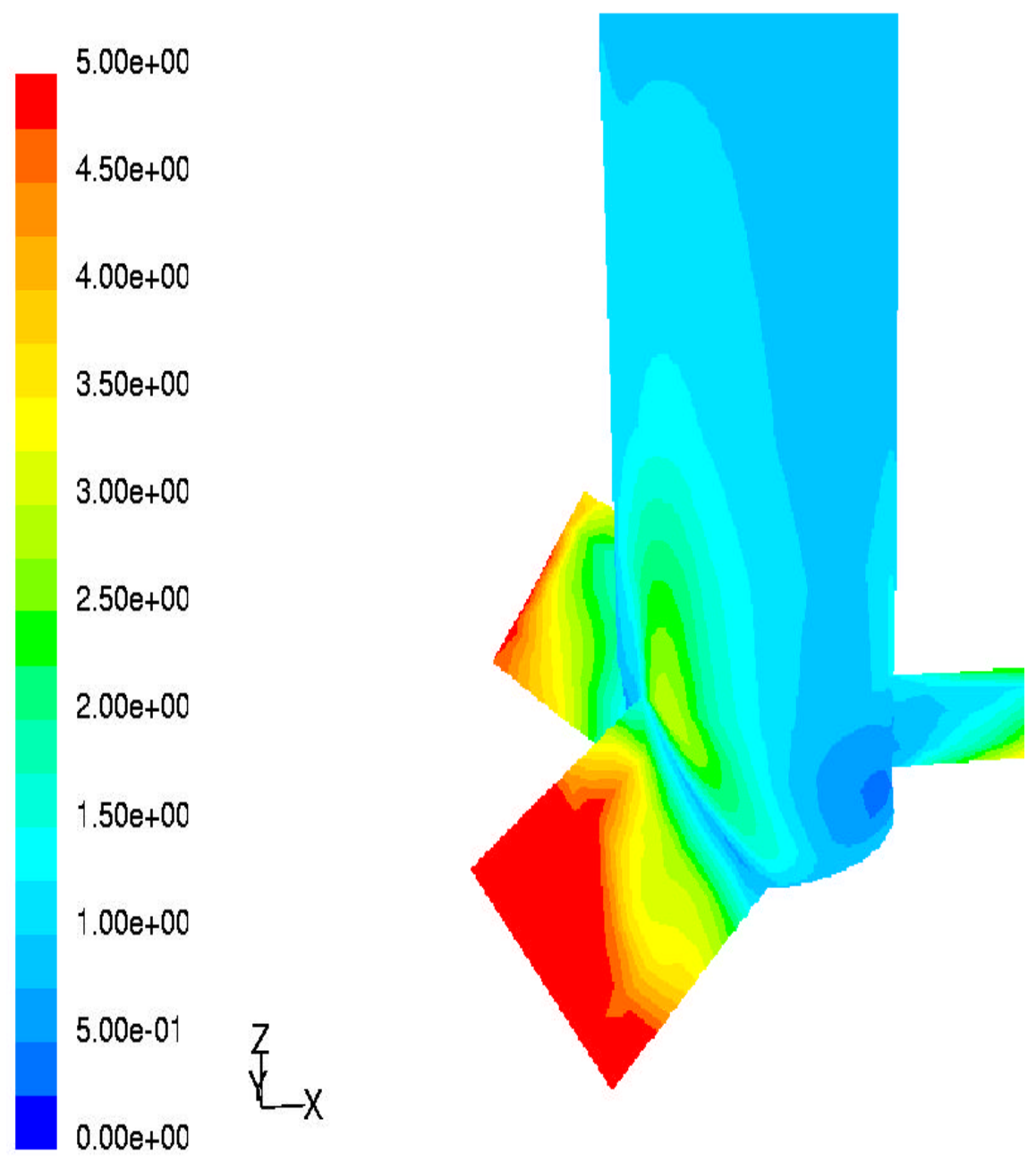

\section{Contours of Wall Shear Stress (pascal)}

\section{FLUENT 6.C}

Figure 21. Wall shear distributions on the surface of the agitator blade and its rotational axis located near the tank bottom for the reference case shown in Table 1 (12-in tank level and $500 \mathrm{rpm}$ agitator speed) 


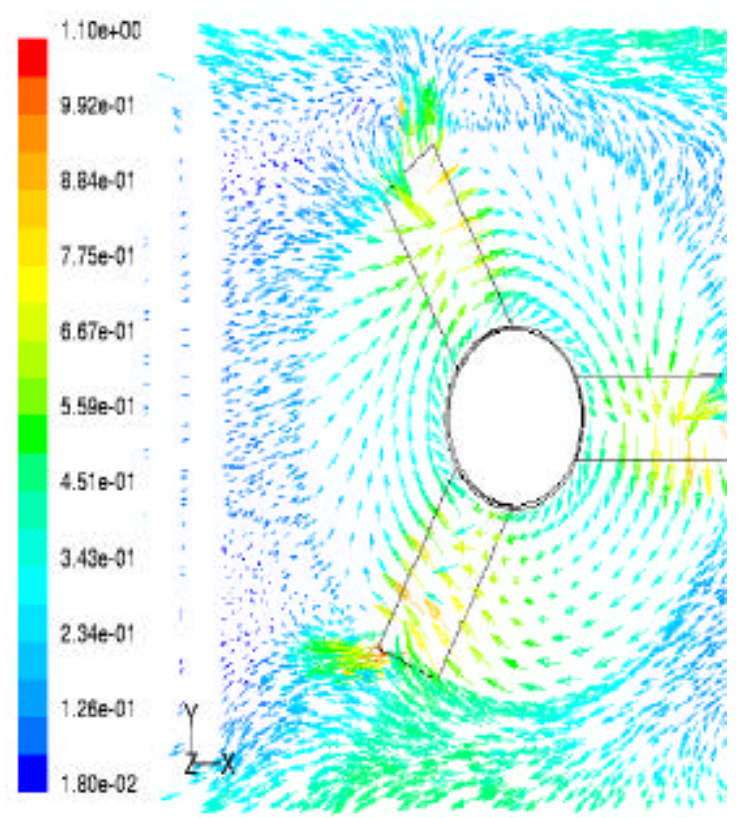

Velocity Vectors Colored By Velocity Magnitude (ms)

(Flow patterns)

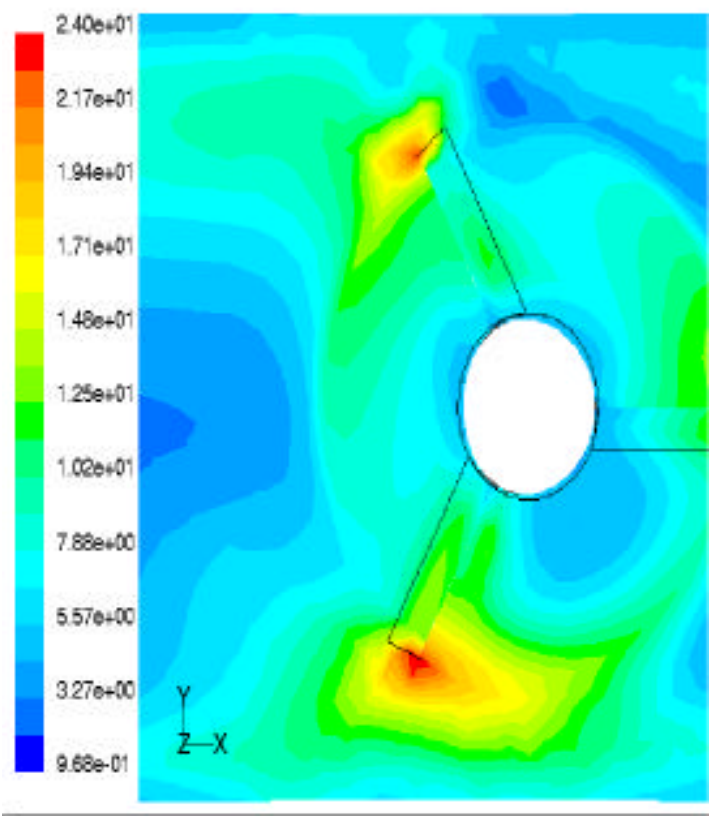

Contours of Turbulence Intens:y $(\%)$

(Turbulence intensity)

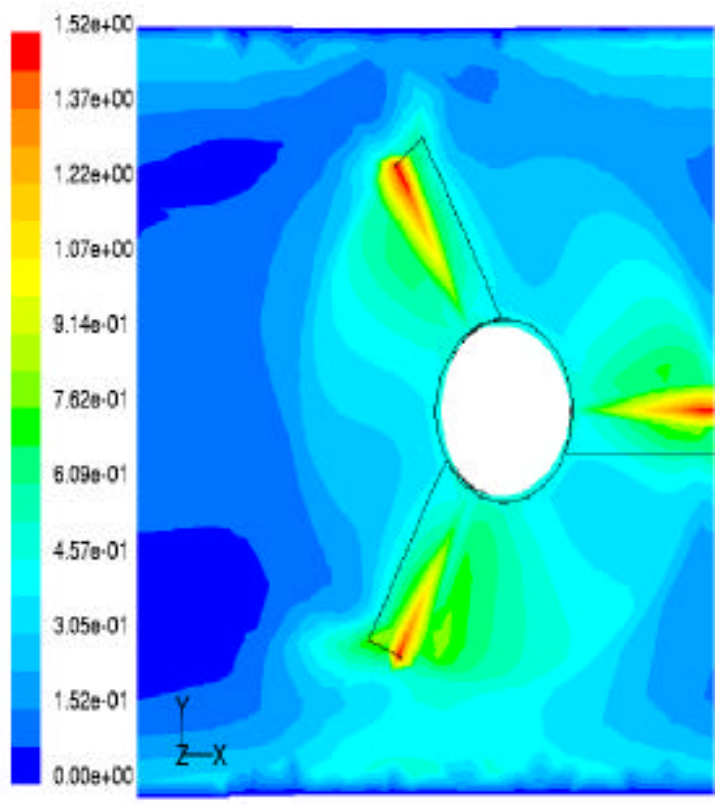

Contours of Velocity Nagnitude (m/s)

FUENT 6 C

(Velocity magnitude)

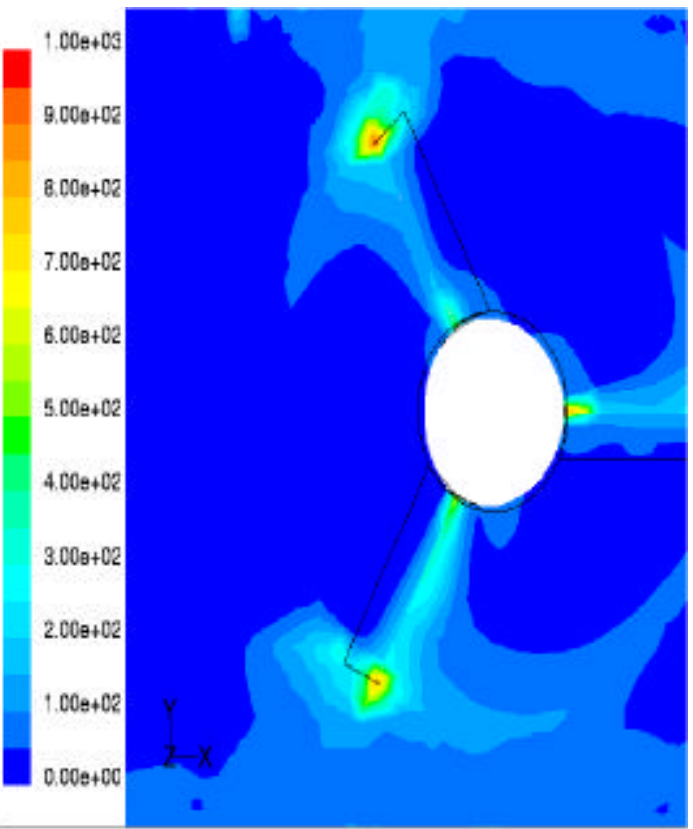

Contours of Vortcity Magnitude

FLLNN $6 \mathrm{C}$

(Vorticity magnitude)

Figure 22. Contour plots for some physical parameters due to the clockwise rotations of the agitator on the plane crossing the vertical center of the agitator for the reference case (500 rpm agitator speed and 12-inch tank level) 


\section{Page: $\quad 34$ of 56}

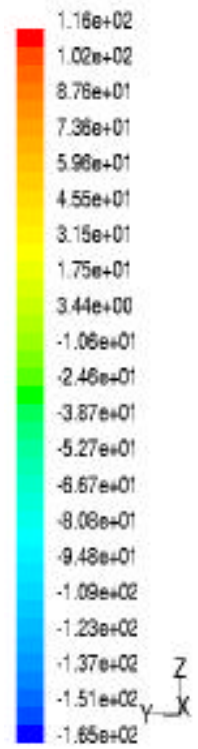

\footnotetext{
Contours of Static Pressure (pascal)
}

(Pressure distributions)
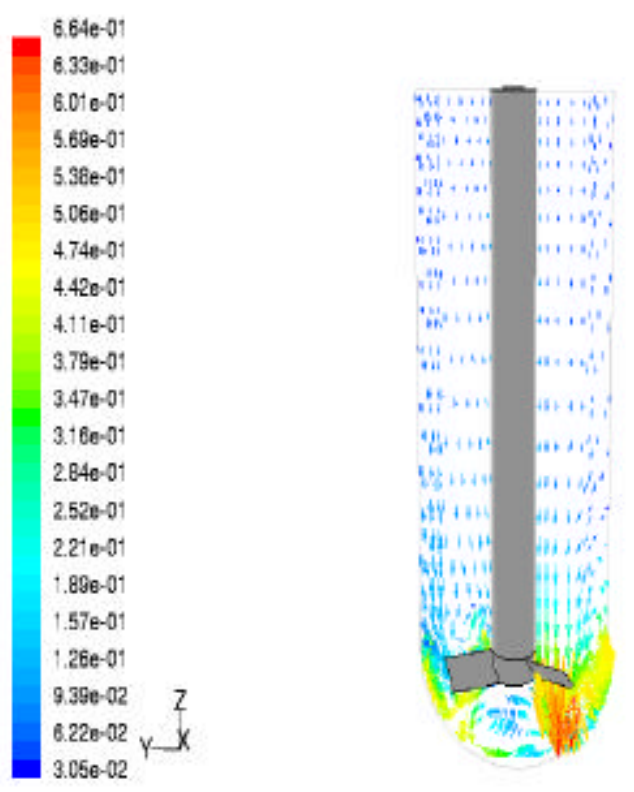

Velocey Vectors Colored By Velocity Magnitude (m/s)

FLLUENT 6C

(Flow circulation pattern)
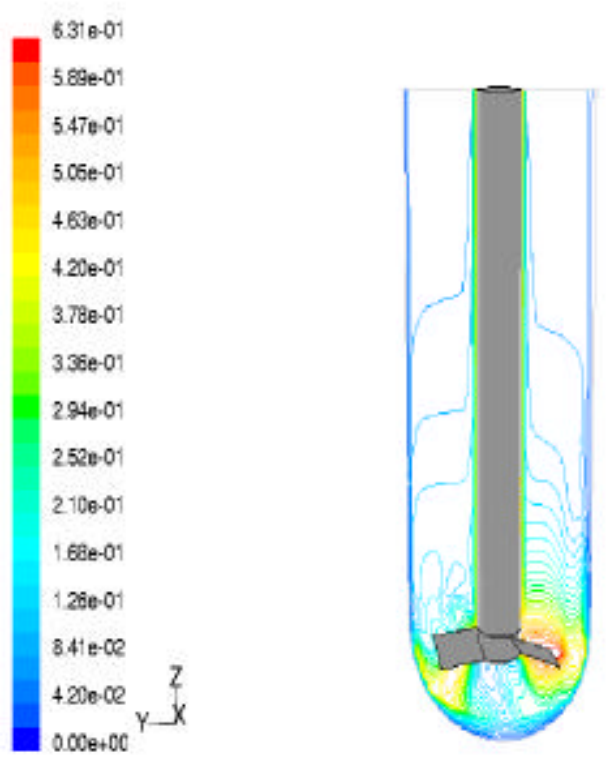

Contours of Velocity Magnitude (m/s)

FULNT 6C

(Velocity distributions)
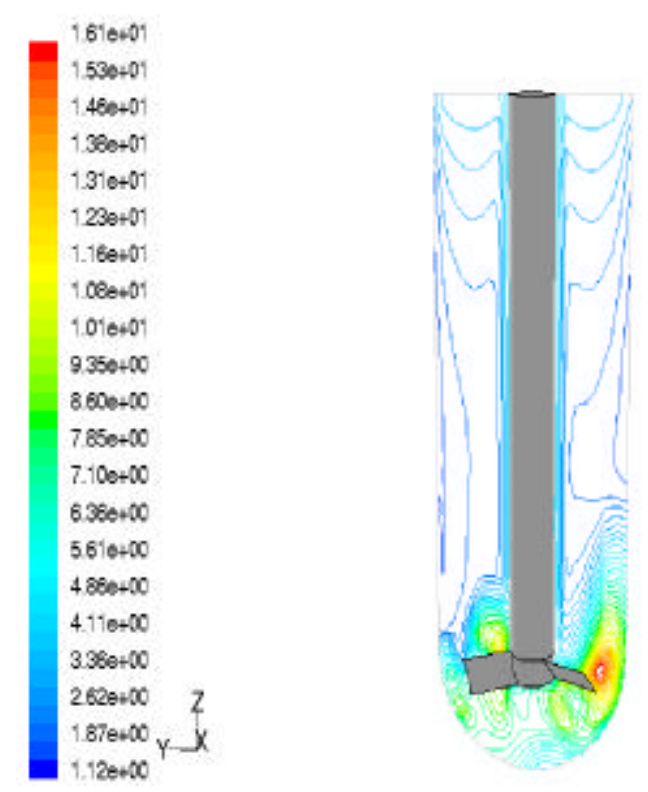

Contours of Turbulence Intens:y (\%)

FUUENT $6 \mathrm{C}$

(Turbulence intensity distributions)

Figure 23. Distributions of physical parameters associated with flow circulation pattern and mixing characteristics for the plane crossing the center of the 500 rpm mixing agitator submerged in the tank with 8.5-inch liquid level. 


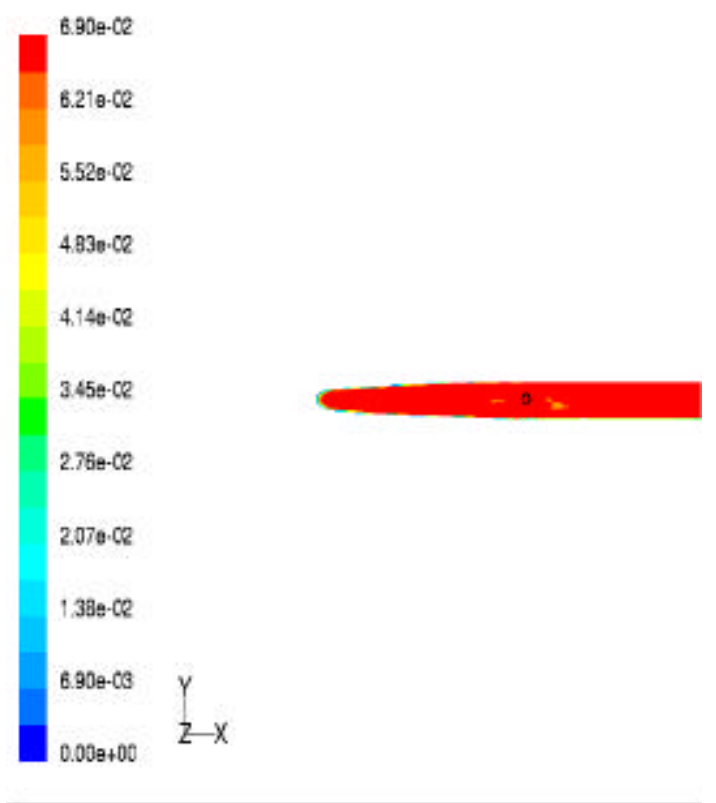

Contours of Velocity Magnitude (m/s)
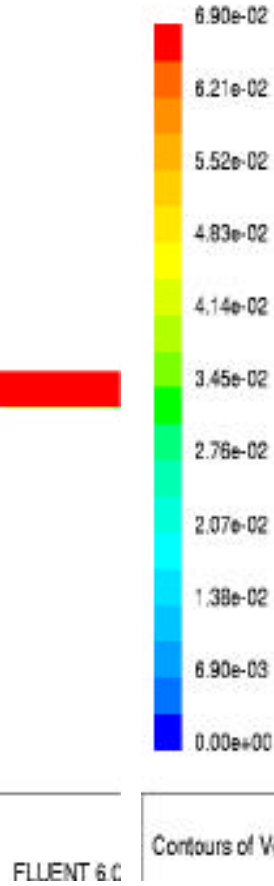

5.520 .02

$4.83 \mathrm{e} \cdot 02$

4.140 .02

$3.45 \div-0 R$

$2.76 \leq-02$

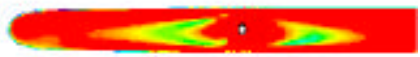

2.070 .02

$1386-02$

$6.90 \mathrm{e}-03$

$z x$

Cortours of Veboty Magntude (m/s)

(elevation $=2.6$ inches $)$

(elevation $=0.5$ inches)

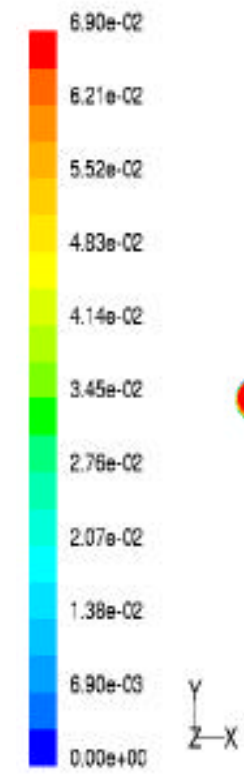

Contours of Velocity Magnitude (mis)

FUENT 6 C

(elevation $=6.7$ inches)

Figure 24. Velocity contour plots for the horizontal planes corresponding to different elevations above the tank bottom for $500 \mathrm{rpm}$ agitator speed and the 8.5inch tank level 


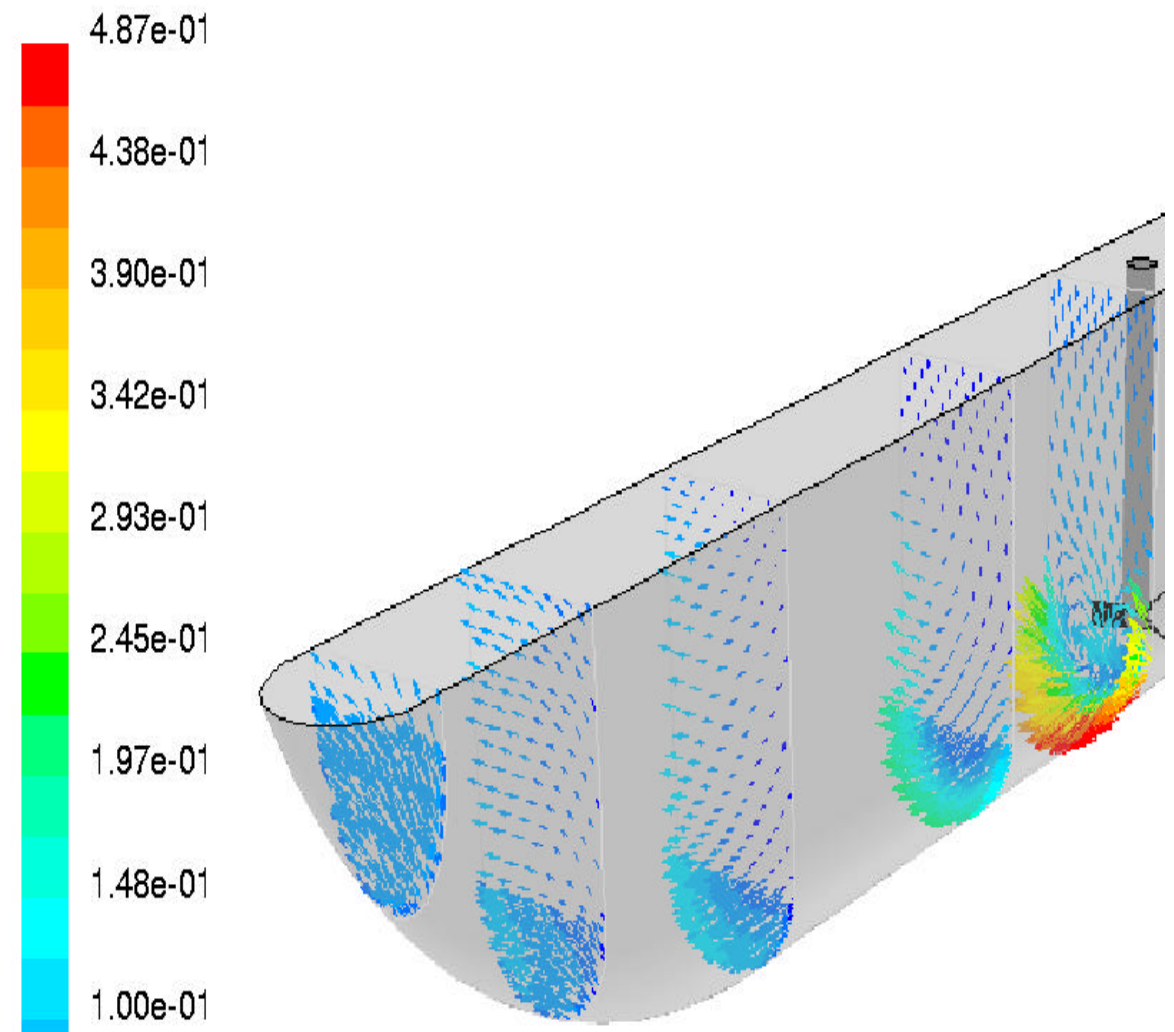

$5.16 \mathrm{e}-02$<smiles>[Y]CC([Y])([3H])[3H]</smiles>

$3.30 \mathrm{e}-03$

Velocity Vectors Colored By Velocity Magnitude $(\mathrm{m} / \mathrm{s})$

\section{FLUENT $6 . \mathrm{C}$}

Figure 25. Velocity vector plots for the planes at several different distances $(x=2 ", 8.25$ ", 17.5 ", 23.5", and 27.5") from the agitator located at the center of the flat tank (500 rpm agitator speed with 8.5-inch tank level). 


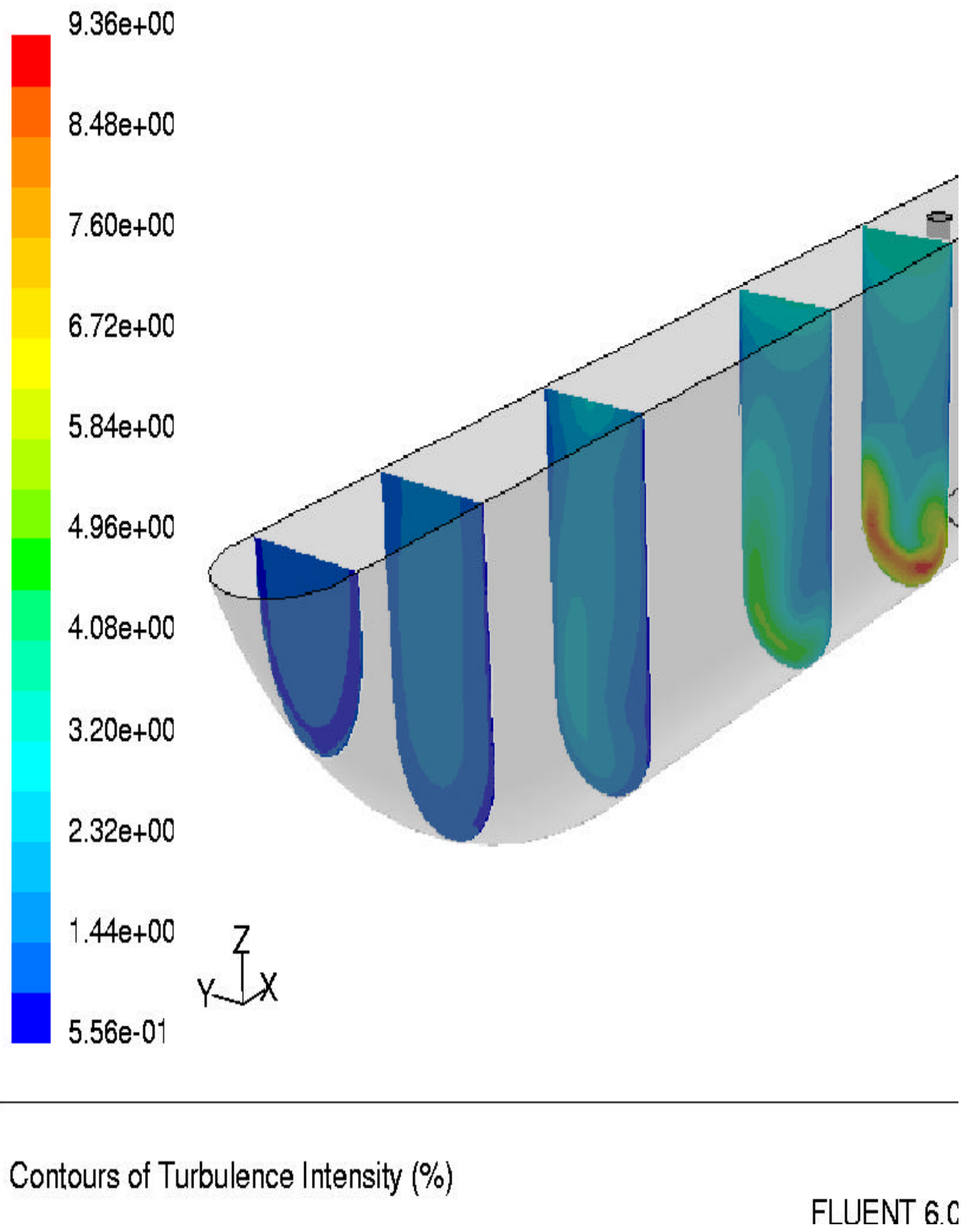

Figure 26. Turbulence intensity contour plots for the planes at several different distances ( $x=2$ ", 8.25", 17.5", 23.5", and 27.5") from the agitator located at the center of the flat tank (agitator speed $=500 \mathrm{rpm}$ and 8.5-inch tank level) 


\section{Page: $\quad 38$ of 56}

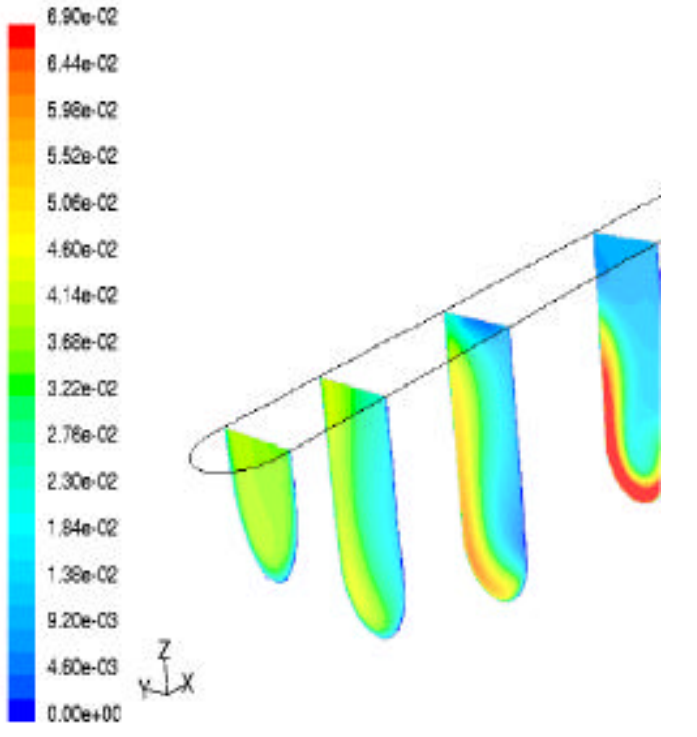

Comiours of Veiccty Magnitute (m/s)

(agitator speed $=300 \mathrm{rpm}$ )
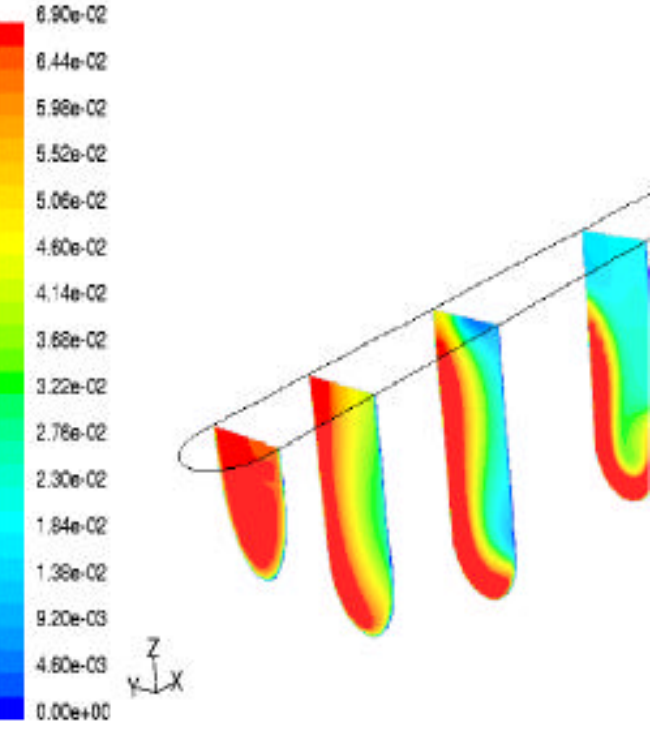

Contours of Veccty Vagniuste (m/s)

FUUENT B. C

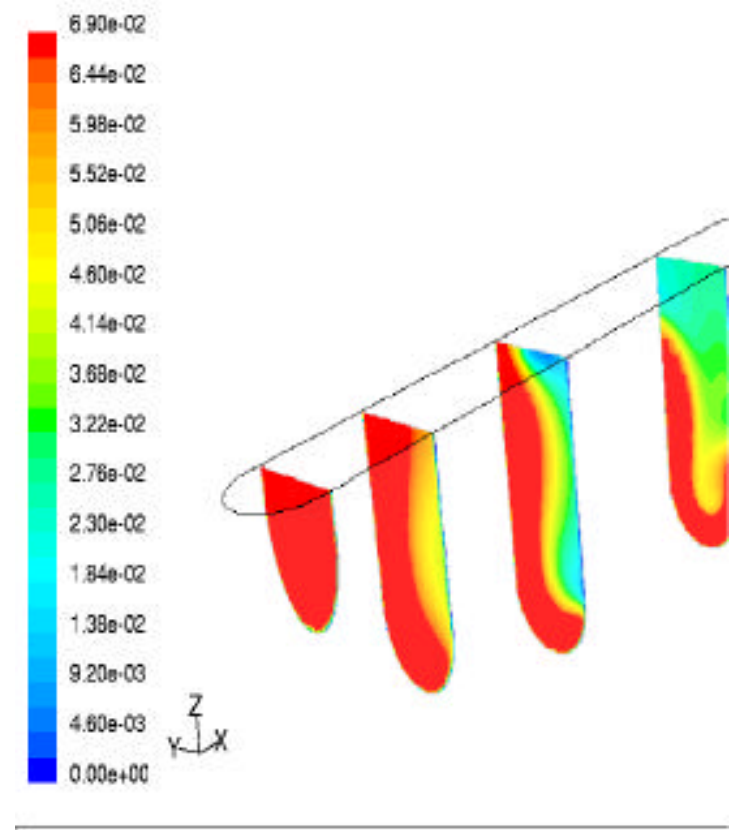

Contours of Velocity Magritude (m/s)

FUENT 6 C

(agitator speed $=700 \mathrm{rpm}$ )

Figure 27. Velocity contour plots for the planes at several different distances $(x=2 "$, 8.25", 17.5", 23.5", and 27.5") from the agitator located at the center of the flat tank with 8.5-inch liquid level. 

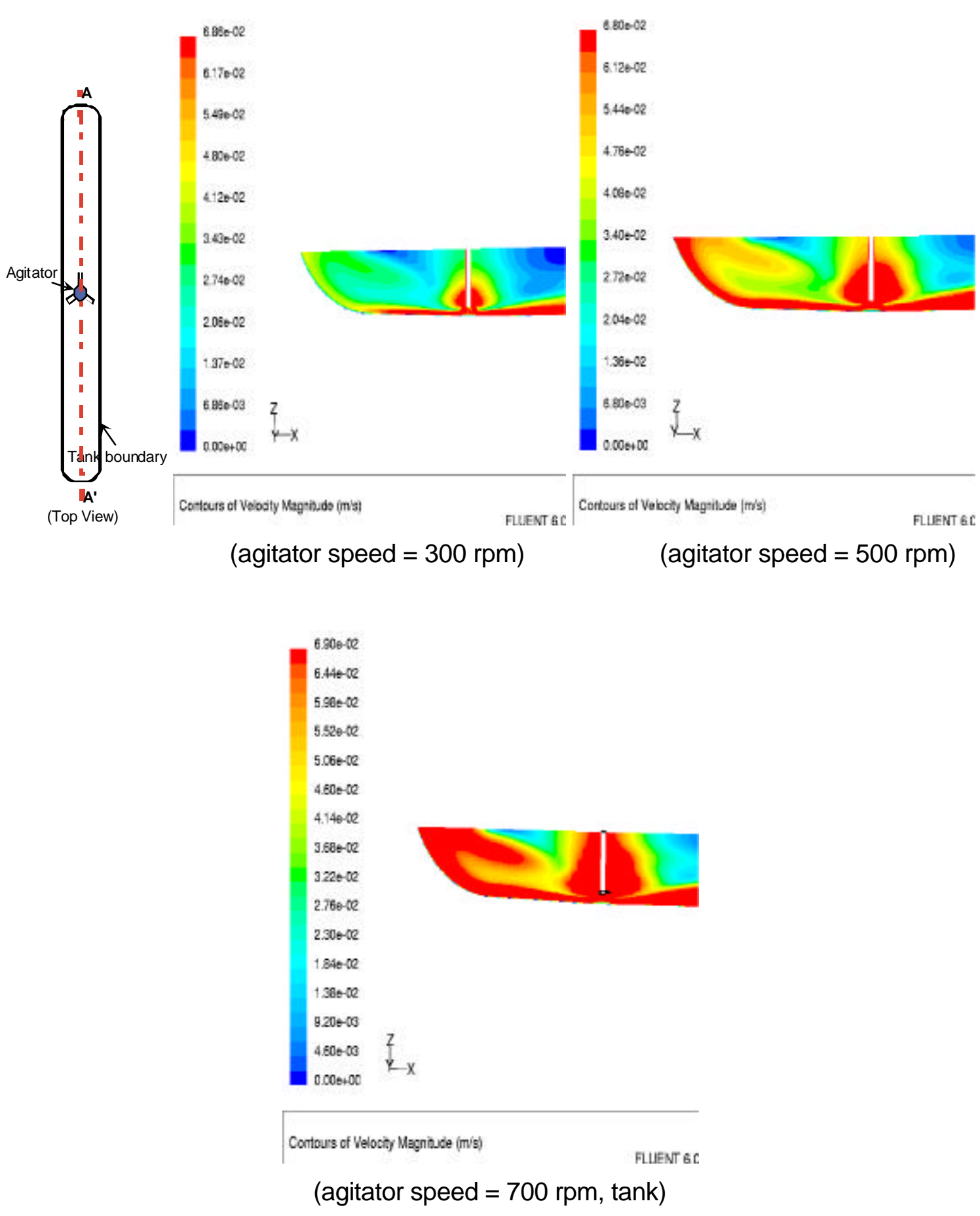

Figure 28. Velocity contour plots at the vertical plane crossing the mixer (line A-A') for various different agitator speeds in the flat tank filled with 8.5-inch liquid level 
Date: $\quad 07 / 24 / 02$

Page: $\quad 40$ of 56

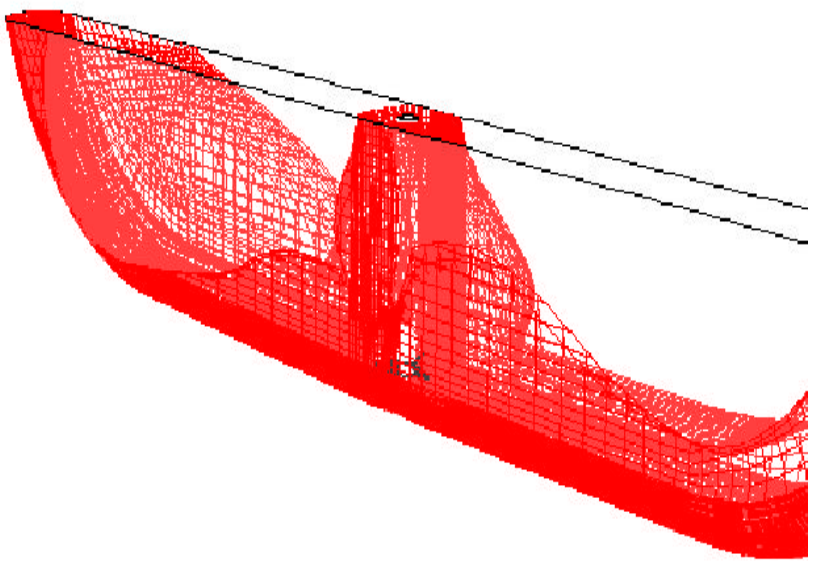

$z_{x}$

Figure 29. Three-dimensional velocity contour plots showing the fluid domain to be higher than minimum critical velocity $(0.225 \mathrm{ft} / \mathrm{sec}$ for 20 -micron particle) for flat tank with 600 rpm agitator speed and 8.5-inch tank level. 

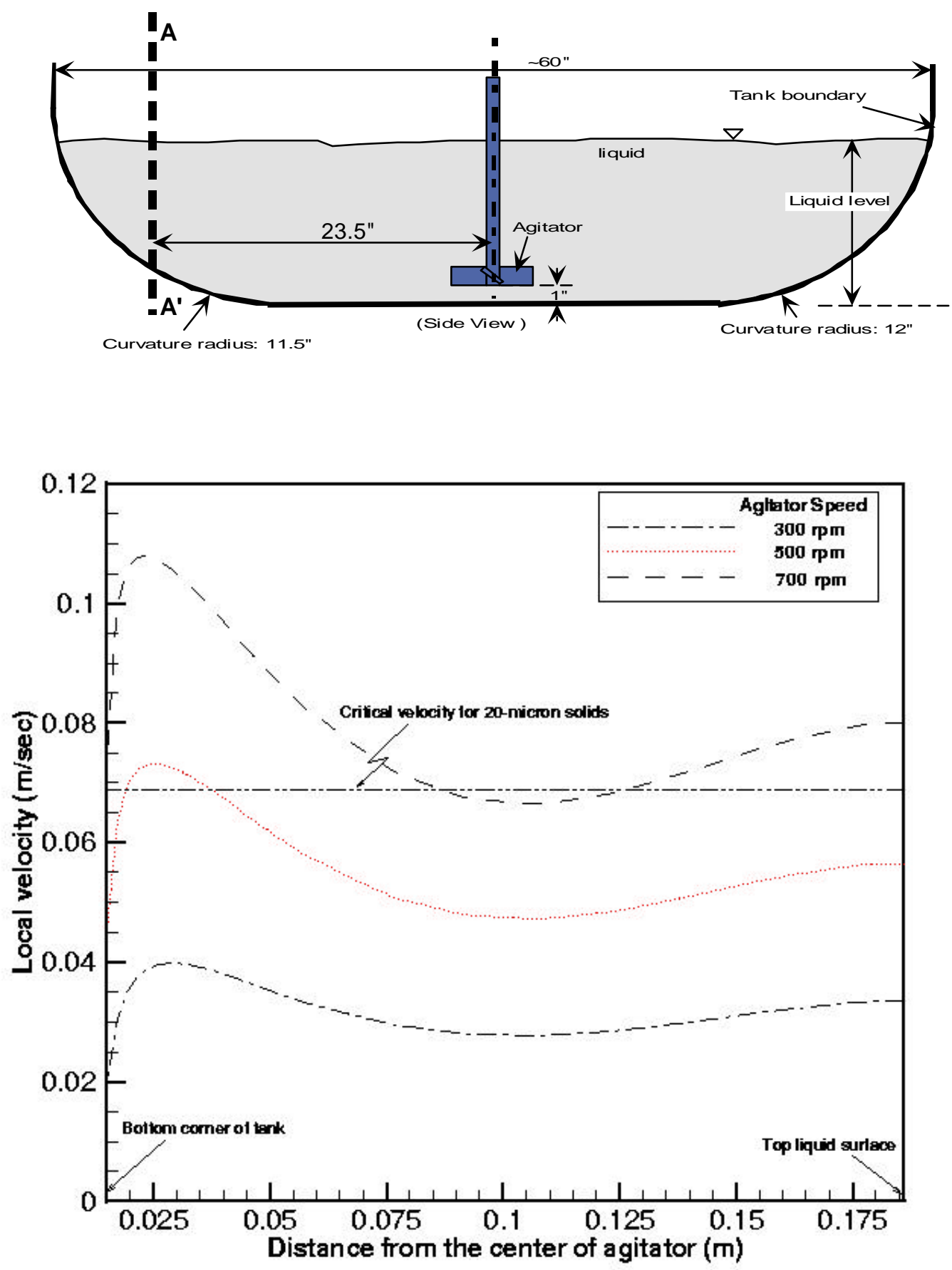

Figure 30. Velocity distributions at the vertical plane crossing the line A-A' for three different agitator speeds in the flat tank filled with 8.5-inch liquid level 


\section{Page: $\quad 42$ of 56}
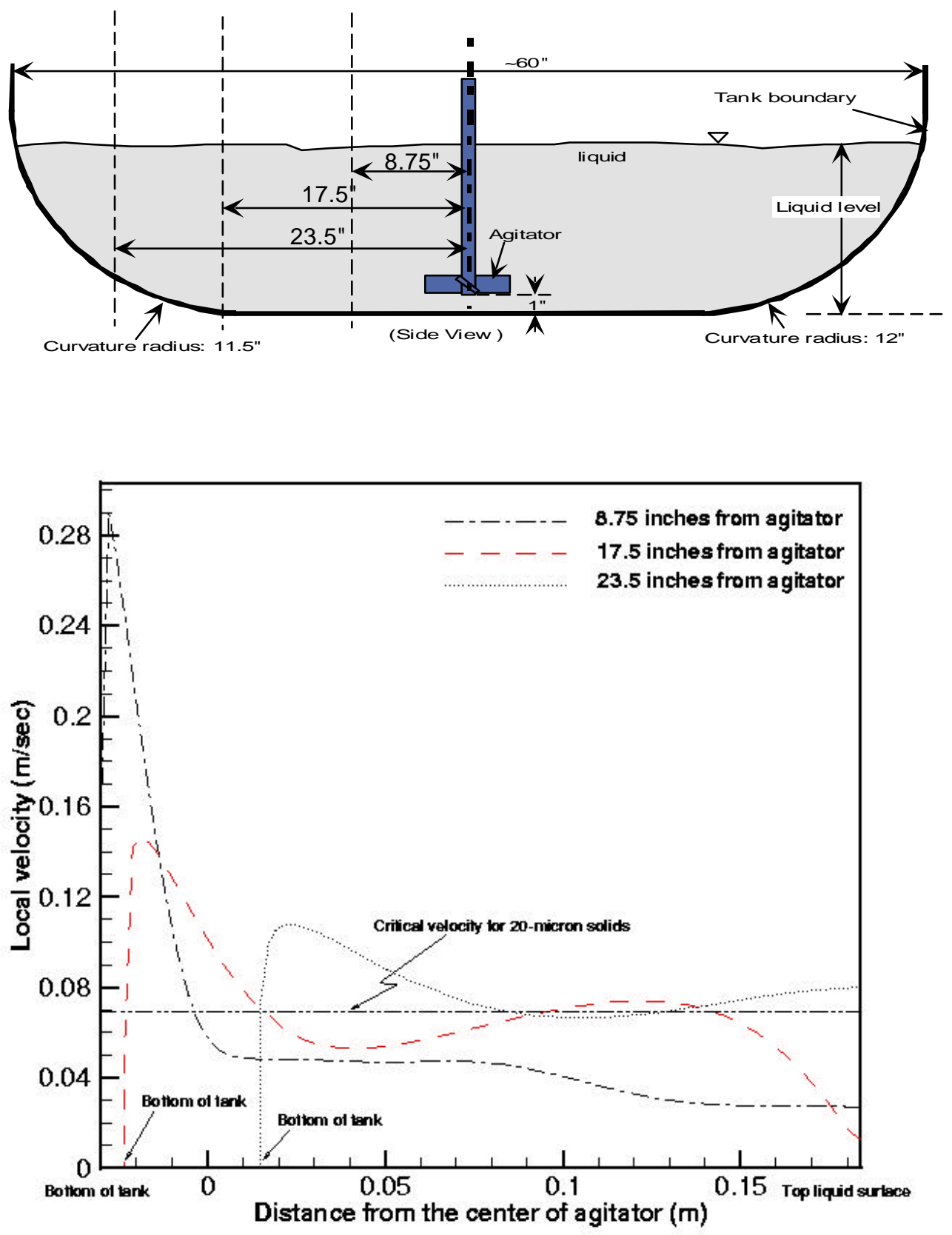

Figure 31. Velocity distributions along the vertical direction from the tank bottom for various horizontal distances from the 700-rpm agitator submerged in the flat tank filled with 8.5-inch liquid level 

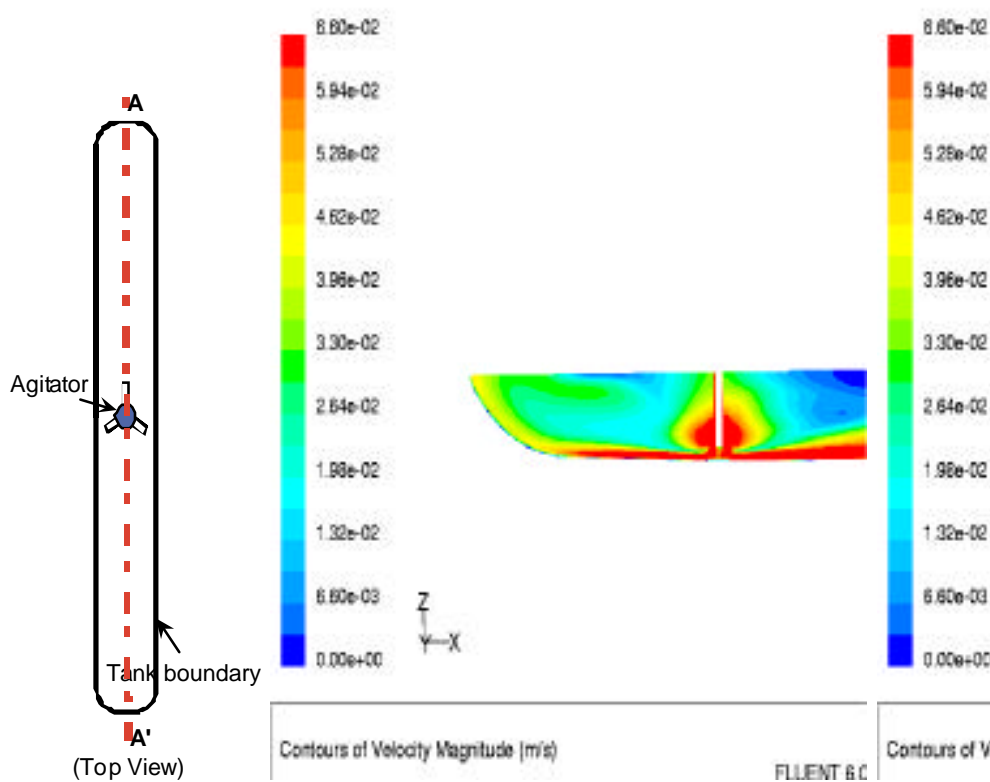

$5250 \cdot 00$

$4626-02$

$4620-5 e$

$3960-02$

3960.06

390002

$2 B 4002$

$1.960-02$

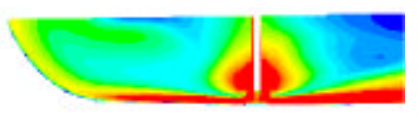

$330 \mathrm{e}-0 \mathrm{e}$

264008

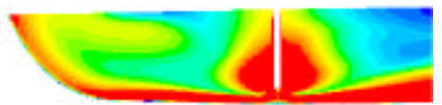

$130=02$

+920-00

$132 \mathrm{ar}$

B B B003

$\sum_{y-x}$

6800000

$\sum_{y-x}^{Z}$

Cotiours of Volocity Magnituse (mis)

Cottous of Velocidy Nagntude (mial

PUENT \&C

(agitator speed $=300 \mathrm{rpm}$ )

(agitator speed $=400 \mathrm{rpm}$ )
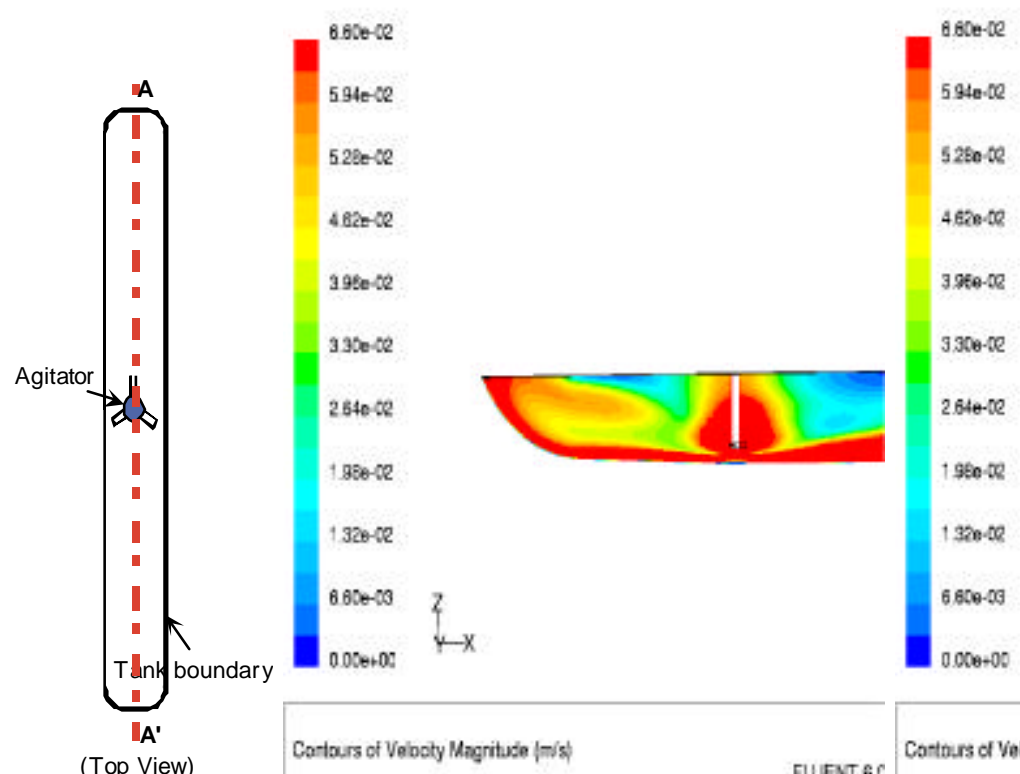

4620.08

$3960-08$

$3,300-96$

$2.640-06$

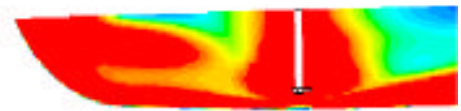

1960.08

132000

$6.800 \cdot 03$

$0.000+00 t_{k}^{k}$

(Top View)

(agitator speed $=500 \mathrm{rpm}$ )

(agitator speed $=700 \mathrm{rpm}$ )

Figure 32. Velocity contour plots at the vertical plane crossing the mixer (line A-A') for various different agitator speeds in the flat tank filled with 7 -inch liquid level 

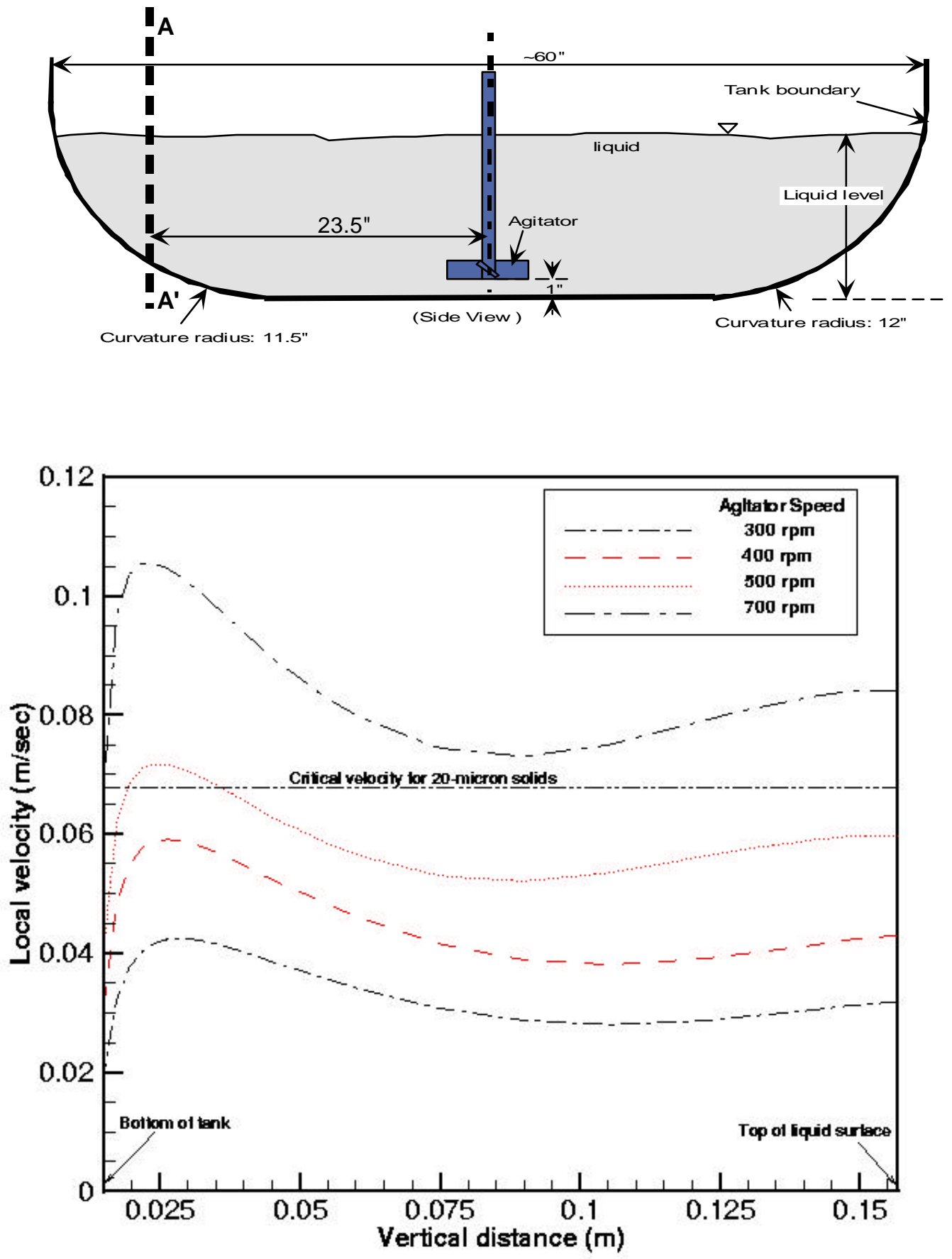

Figure 33. Velocity distributions along the vertical line A-A' for various different agitator speeds at the horizontal distance of 23.5 inches from the agitator submerged in the flat tank filled with 7-inch liquid level 

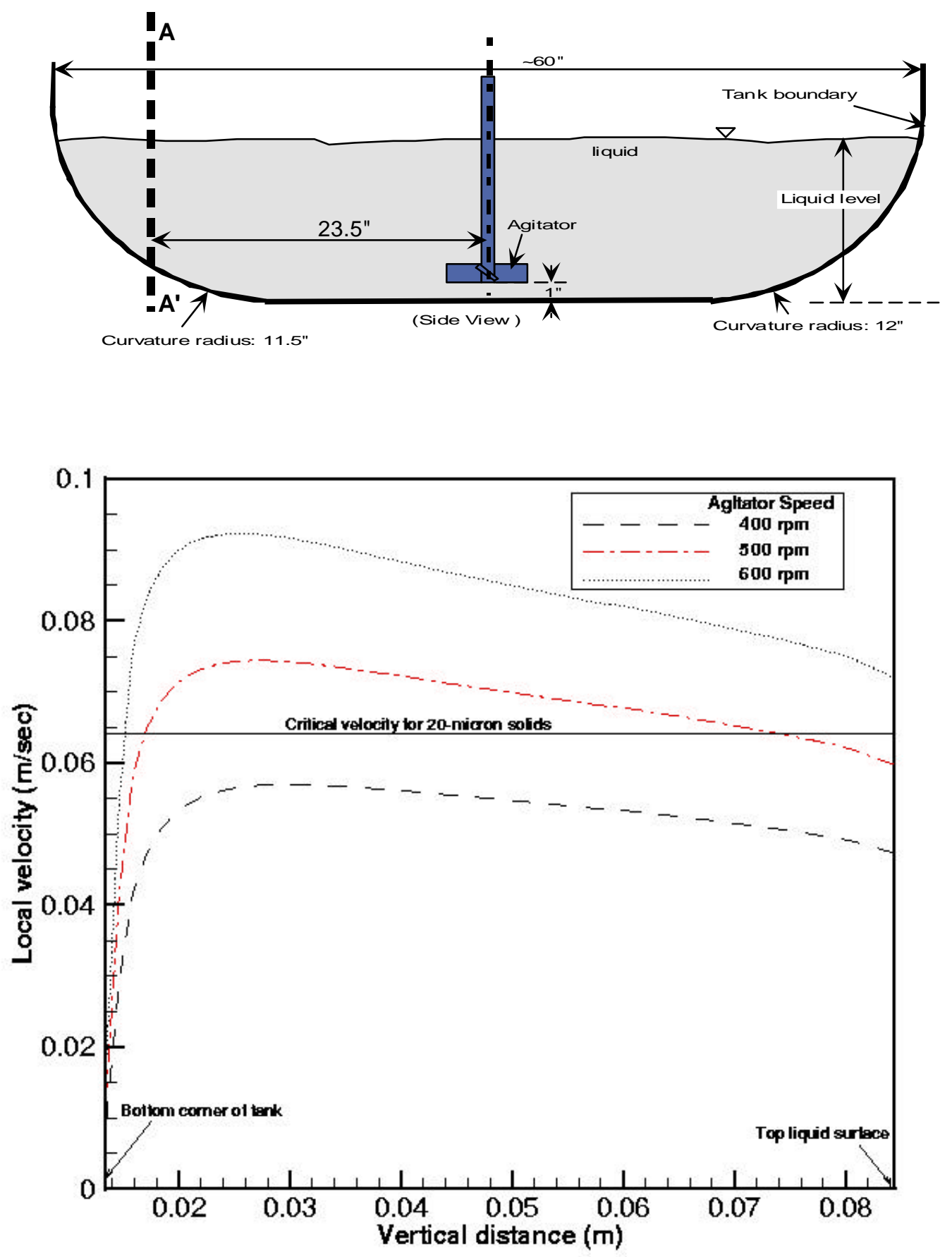

Figure 34. Velocity distributions along the vertical line A-A' for two different agitator speeds at the horizontal distance of 23.5 inches from the agitator submerged in the flat tank filled with 4.5-inch liquid level 


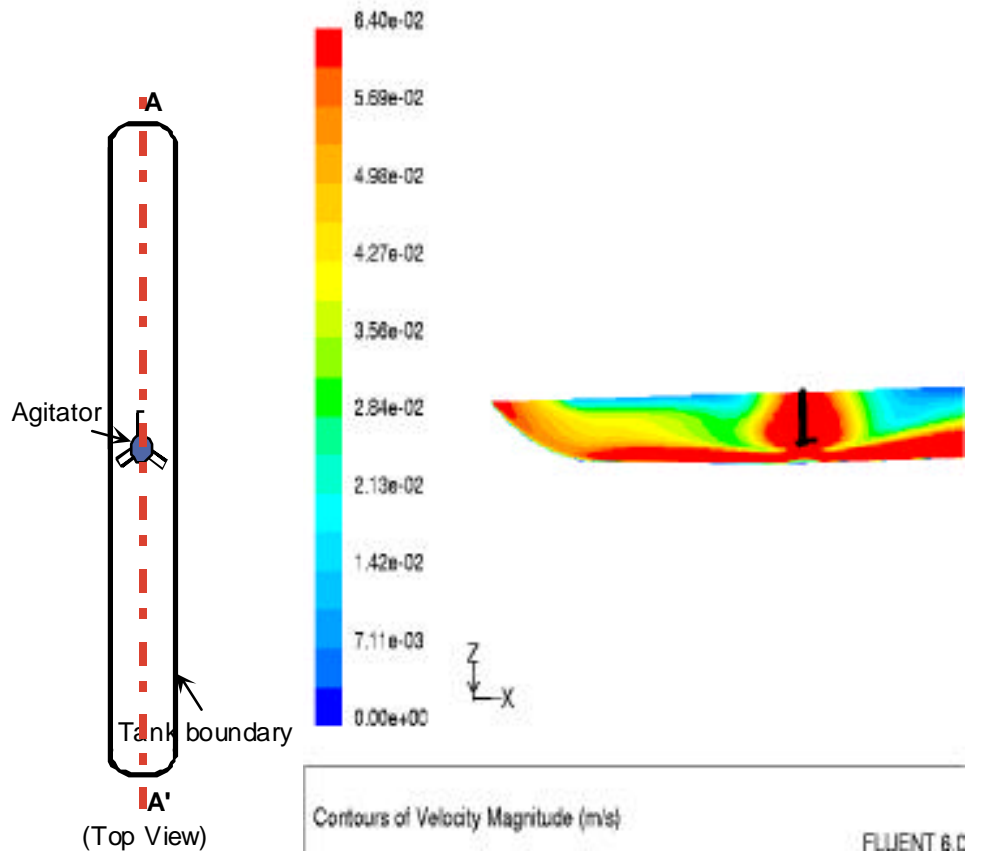

(agitator speed $=400$ rpm)

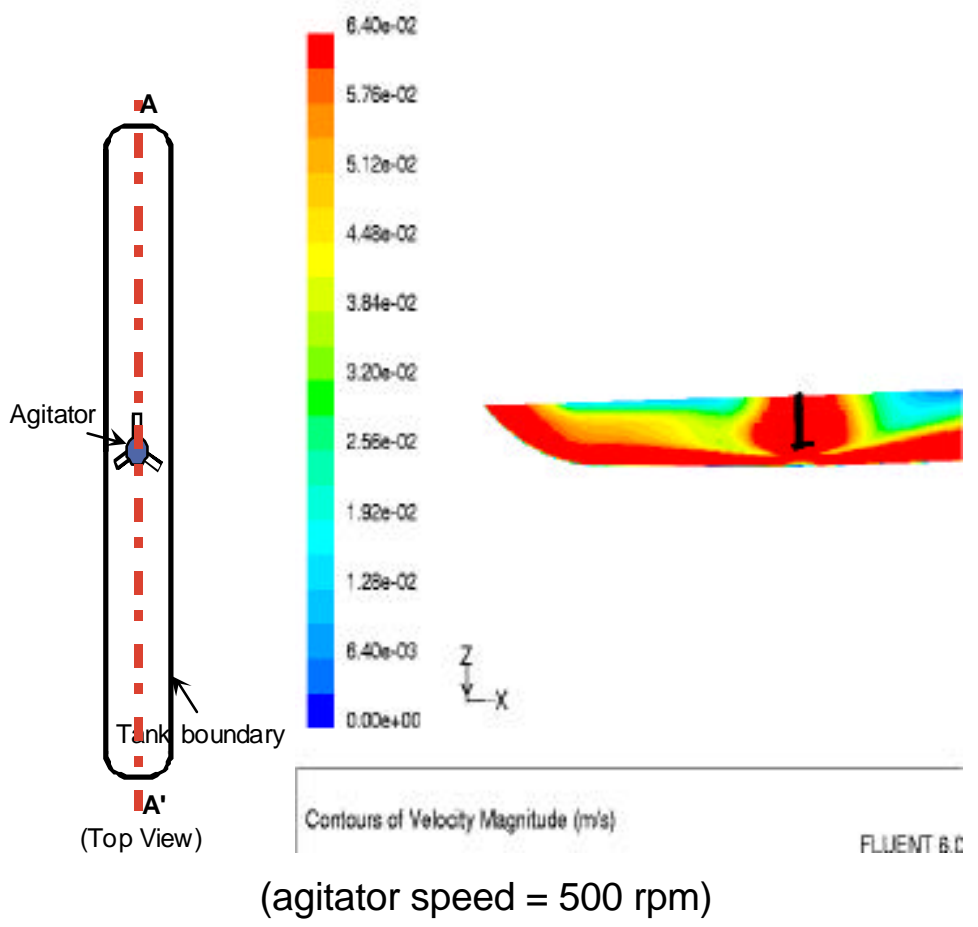

Figure 35. Velocity contour plots at the vertical plane crossing the mixer (line A-A') for two different agitator speeds in the flat tank filled with 4.5-inch liquid level 

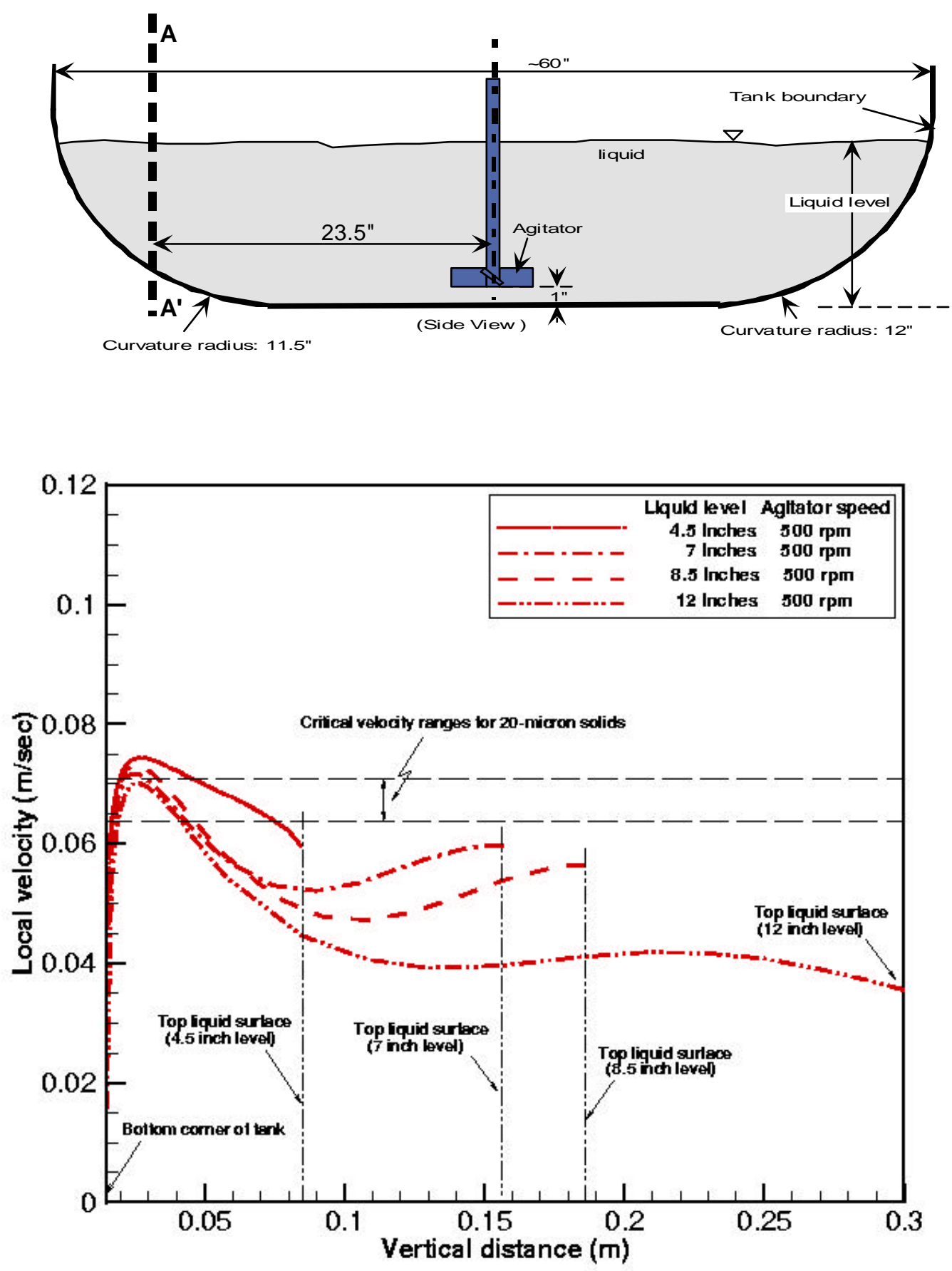

Figure 36. Velocity distributions along the vertical line A-A' for $500 \mathrm{rpm}$ agitator speed at the horizontal distance of 23.5 inches from the agitator submerged in the flat tank filled with various liquid levels 

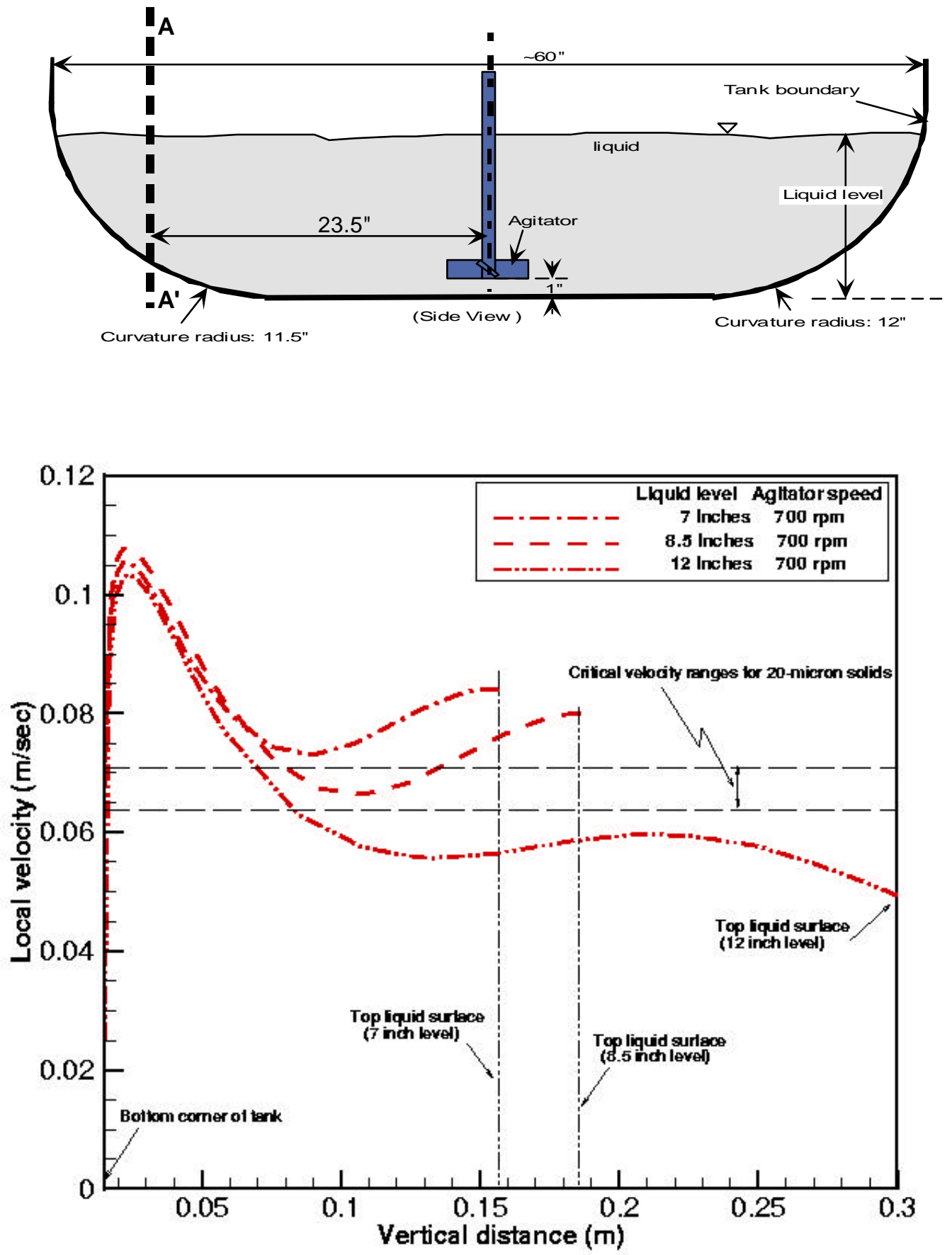

Figure 37. Velocity distributions along the vertical line A-A' for $700 \mathrm{rpm}$ agitator speed at the horizontal distance of 23.5 inches from the agitator submerged in the flat tank filled with various liquid levels 


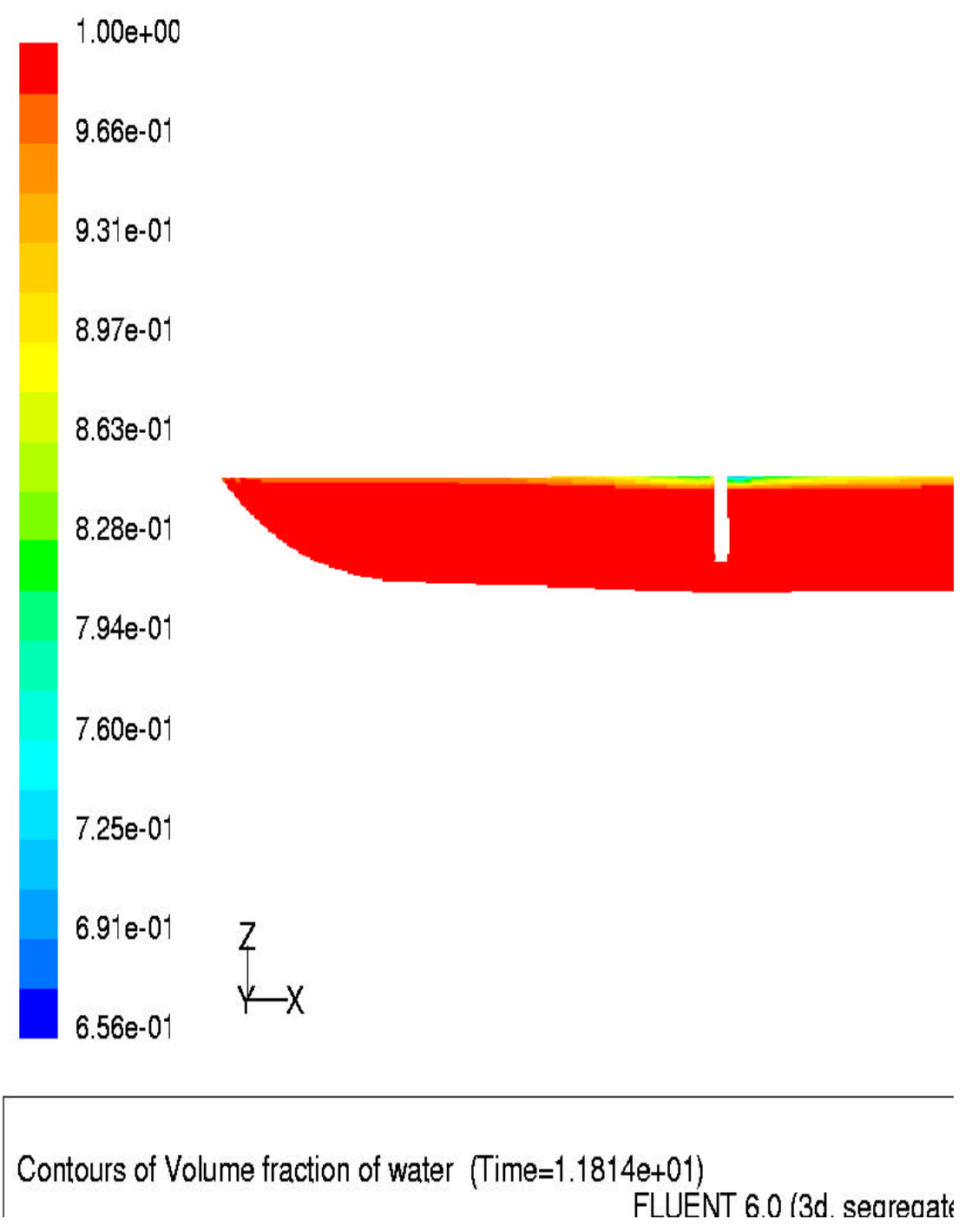

Figure 38. Typical transient results for water volume fractions in 4.5-inch tank level with $400 \mathrm{rpm}$ agitator speed considering free surface motion 
Report: WSRC-TR-2002-00219

Date: $\quad 07 / 24 / 02$

Page: $\quad 50$ of 56
WESTINGHOUSE SAVANNAH RIVER COMPANY

AGITATOR MIXING ANALYSIS IN A HB-LINE FLAT TANK

Table 6. Modeling results for various tank levels and pump speeds compared with critical velocities

\begin{tabular}{|c|c|c|c|c|}
\hline $\begin{array}{c}\text { Tank } \\
\text { levels } \\
\text { (inches) }\end{array}$ & $\begin{array}{l}\text { Tank fluid } \\
\text { volume } \\
\text { (liter) }\end{array}$ & $\begin{array}{l}\text { Pump } \\
\text { speed } \\
\text { (rpm) }\end{array}$ & $\begin{array}{l}\text { Average local velocity at } \\
\text { bottom corner of flat tank } \\
\text { (m/sec) }\end{array}$ & $\begin{array}{l}\text { Critical } \\
\text { velocity } \\
\text { (m/sec) }\end{array}$ \\
\hline \multirow{4}{*}{4.5} & \multirow{4}{*}{$\sim 10$} & 300 & 0.0373 & \multirow{4}{*}{0.064} \\
\hline & & 400 & 0.0527 & \\
\hline & & 500 & 0.0686 & \\
\hline & & 600 & 0.0846 & \\
\hline \multirow{4}{*}{7} & \multirow{4}{*}{$\sim 19$} & 400 & 0.0511 & \multirow{4}{*}{0.067} \\
\hline & & 500 & 0.0630 & \\
\hline & & 600 & 0.0772 & \\
\hline & & 700 & 0.0915 & \\
\hline \multirow{4}{*}{8.5} & \multirow{4}{*}{$\sim 21$} & 400 & 0.0484 & \multirow{4}{*}{0.069} \\
\hline & & 500 & 0.0626 & \\
\hline & & 600 & 0.0769 & \\
\hline & & 700 & 0.0912 & \\
\hline \multirow{4}{*}{12} & \multirow{4}{*}{$\sim 38$} & 400 & 0.0448 & \multirow{4}{*}{0.071} \\
\hline & & 500 & 0.0578 & \\
\hline & & 600 & 0.0709 & \\
\hline & & 700 & 0.0839 & \\
\hline
\end{tabular}



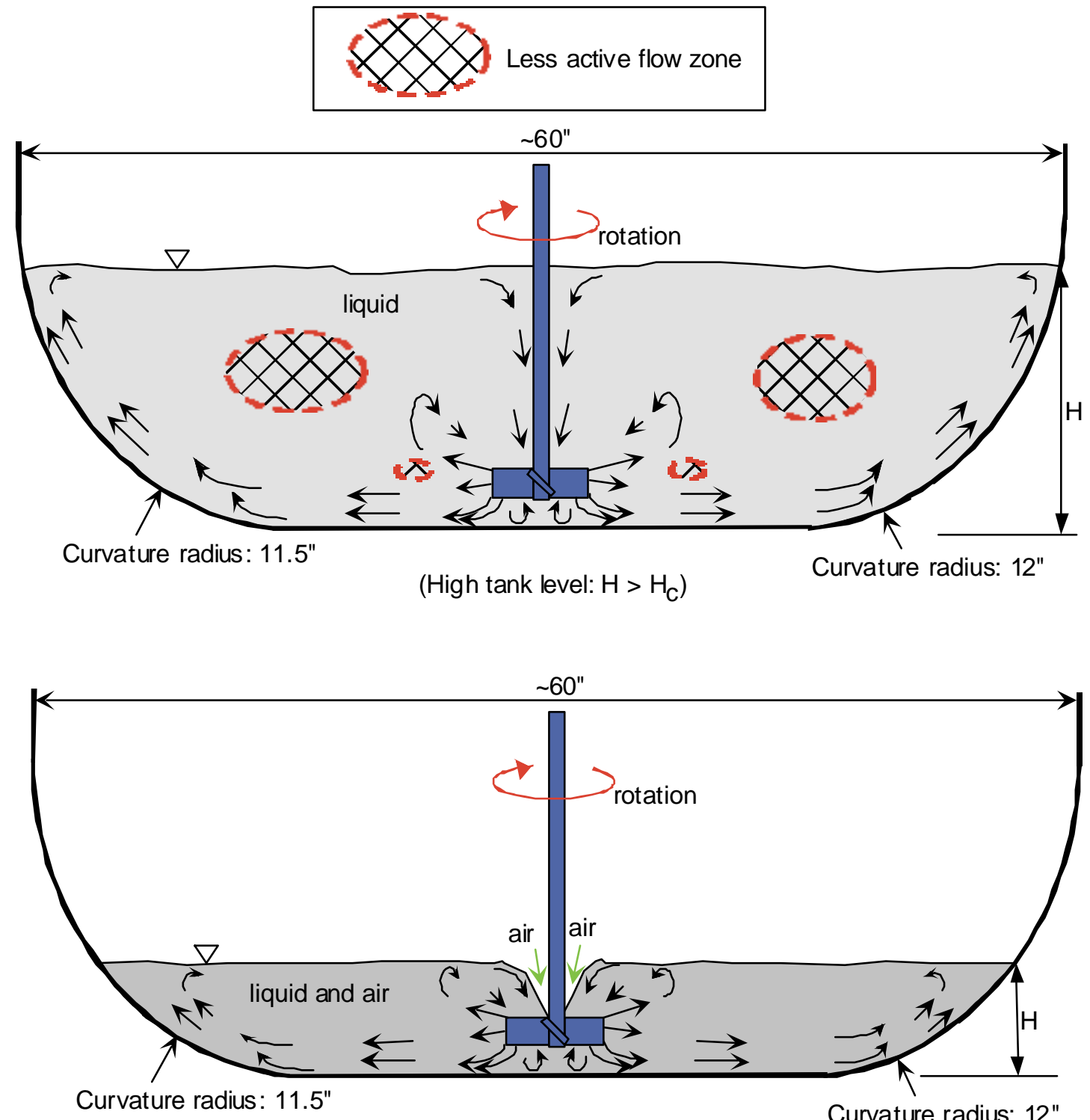

(Low tank level $\left(\mathrm{H}<\mathrm{H}_{\mathrm{C}}\right)$ and high speed: air entrainment)

Figure 39. Flow circulation patterns and characteristics for high and low tank levels with turbulent $45^{\circ}$ pitched agitator submerged. 


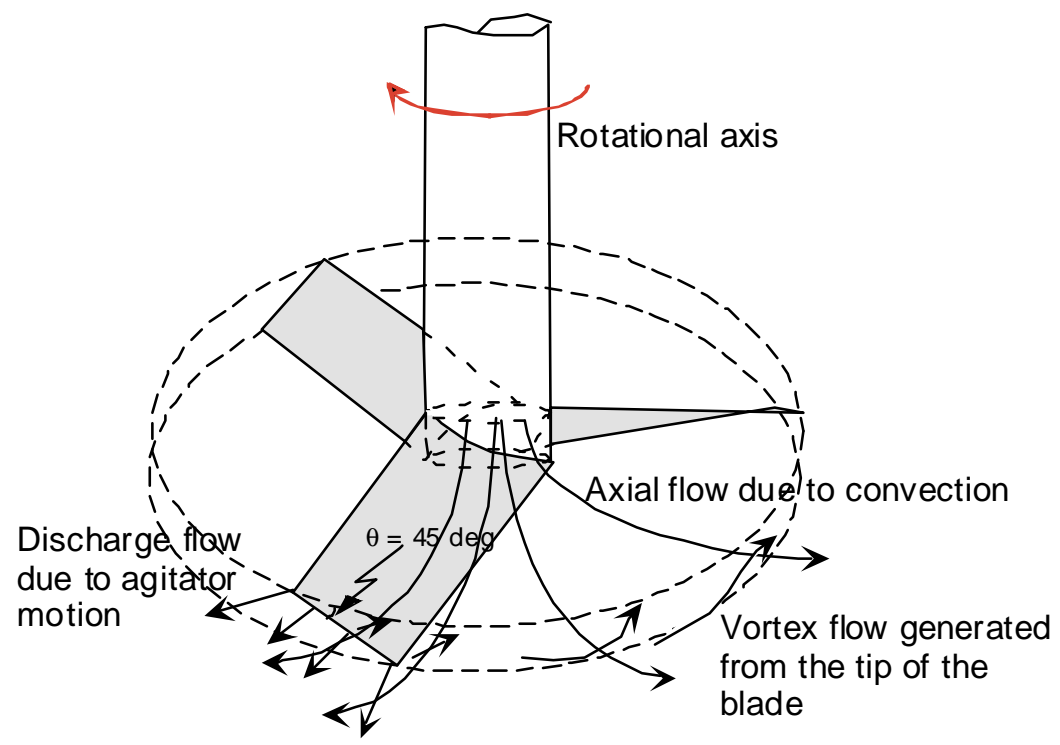

$\left(45^{\circ}\right.$ pitched agitator $)$

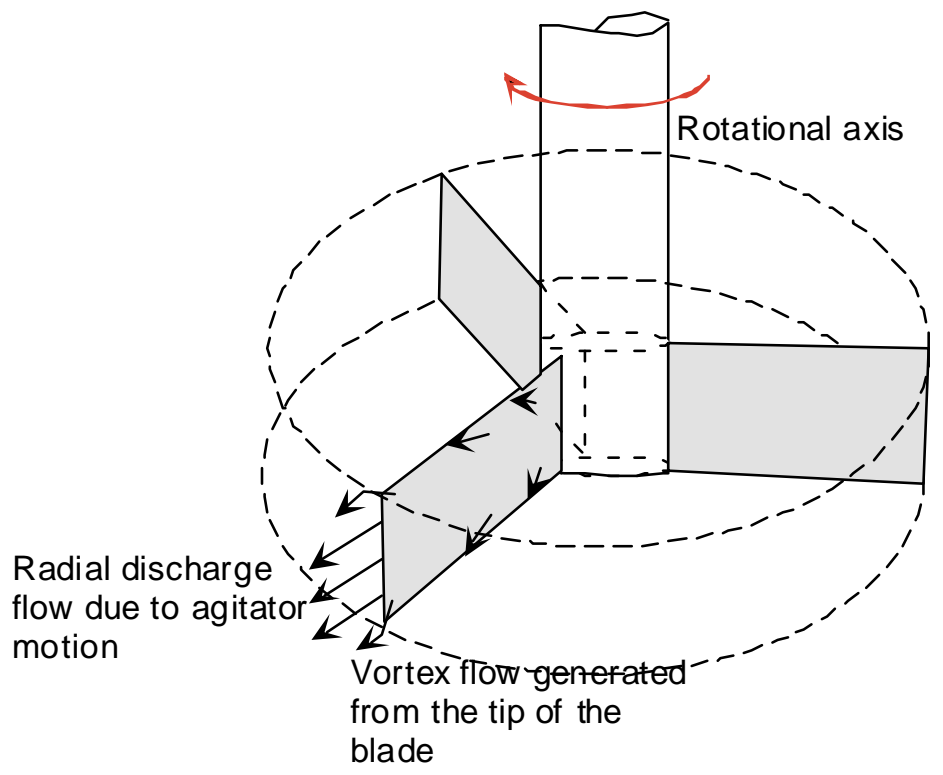

(Rushton-type agitator)

Figure 40. Primary flow components induced by the turbulent agitator submerged in a tank. 


\section{Conclusions}

Flow pattern calculations for potential operating conditions of a 45-deg pitched threeblade agitator in the HB-line flat tank have been performed to assess mixing behavior. Velocity and shear stress criteria were developed to assess the ability of liquid flow to lift and suspend solids deposited on the bottom surface of the tank. The modeling results will help determine acceptable agitator speeds and tank liquid levels to ensure suspension of solid particles deposited during precipitation operations.

Four different tank levels were evaluated: 4.5, 7.0, 8.5, and 12 inches, corresponding to $10,19,21$, and 38 liters, respectively. A three-dimensional CFD approach was used with a two-equation turbulence model and multiple reference frames. Free surface motion was assumed to be negligible compared to forced convective motion for the operating conditions evaluated. The top liquid surface was assumed to be stationary at atmospheric pressure. No-slip boundary conditions were used at the blade surface and the tank walls. Rotational motion of the agitator was simulated by using a rotating reference frame with respect to the adjacent fluid media.

At high agitator rotational speeds and low tank levels, a surface vortex usually forms at the center of the rotating fluid due to the centrifugal force from the agitator forcing the fluid out toward the walls. When the surface vortex reaches the agitator blade, air will be entrained into the mixing zone, which is not desirable in terms of mixing performance. The correlations used in this analysis show that the lowest liquid level evaluated, 4.5 inches, is high enough to prevent air entrainment for agitator speeds up to $600 \mathrm{rpm}$.

All the analysis results demonstrate that an agitator speed of about $600 \mathrm{rpm}$ provides adequate solids mixing capability for various tank levels (12, 8.5, 7, and 4.5 inches) for 20-micron solids with a specific gravity of 2.5. For the lowest tank level, 4.5 inches, 500 rpm is sufficient to suspend the solid particles. Table 6 summarizes the results. 
Report: WSRC-TR-2002-00219

Date: $\quad 07 / 24 / 02$

Page: $\quad 54$ of 56
WESTINGHOUSE SAVANNAH RIVER COMPANY

AGITATOR MIXING ANALYSIS IN A HB-LINE FLAT TANK

(This Page Intentionally Left Blank) 


\section{References}

1. Technical Task Request (TTR), NMMD-HTS-2002-028, February 12, 2002.

2. Personal Communications with J. W. Posnick, February, 2002, and e-mail from D. W. Murdoch, February 27, 2002.

3. S. Y. Lee and R. A. Dimenna, "Design Analysis for a Scaled Erosion Test", WSRCTR-2001-00591 / SRT-RPP-2001-00227, April 2002.

4. D. M. Newitt, J. F. Richardson, M. Abbott, and R. B. Turtle, "Hydraulic Conveying of Solids in Horizontal Pipes", Tans. Instn Chem. Engineers, vol. 33, pp. 93-113, 1955.

5. W. H. Graf, Hydraulics of Sediment Transport, McGraw-Hill Book Company, New York, 1971.

6. V. E. Schrock, R. T. Revankar, R. Mannheimer, S. Y. Lee, and C-H Wang, "Critical Flow Through a Small Break on a Large Pipe with Stratified Flow", Lawrence Berkeley National Laboratory, LBL-18386, 1985.

7. FLUENT, Fluent, Inc., Lebanon, New Hampshire, 1998.

8. F. D. Miller and J. H. Rushton, "A Mass Velocity Theory for Liquid Agitation", Ind. And Eng. Chemistry, vol. 36, no. 6, pp. 499-503, 1944.

9. G. Batchelor, "Theory of Homogeneous Turbulence", p. 103, Cambridge University Press, Cambridge, 1959.

10. A. W. Hixson, "Nature and Measure of Agitation", Ind. And Eng. Chemistry, vol. 36, no. 6, pp. 488-496, June 1944.

11. G. B. Tatterson, Fluid Mixing and Gas Dispersion in Agitated tanks, McGraw-Hill, Inc., New York, 1991.

12. R. B. Richard and G. J. Carlson, "Power Absorption and Fluid Properties, Correlation with Equipment Dimensions and Fluid Properties", Chem. Eng. Progress, vol. 43, no. 9, pp. 473-480, 1947.

13. F. A. Zenz and D. F. Othmer, Fluidization and Fluid-Particle Systems, Reinhold Publishing Corp., New York, 1960.

14. W. M. Rohsenow and H. Y. Choi, Heat, Mass, and Momentum Transfer, PrenticeHall, Inc., New Jersey, 1961. 
Report: WSRC-TR-2002-00219

Date: $\quad 07 / 24 / 02$

Page: $\quad 56$ of 56
WESTINGHOUSE SAVANNAH RIVER COMPANY

AGITATOR MIXING ANALYSIS IN A HB-LINE FLAT TANK

(This Page Intentionally Left Blank) 